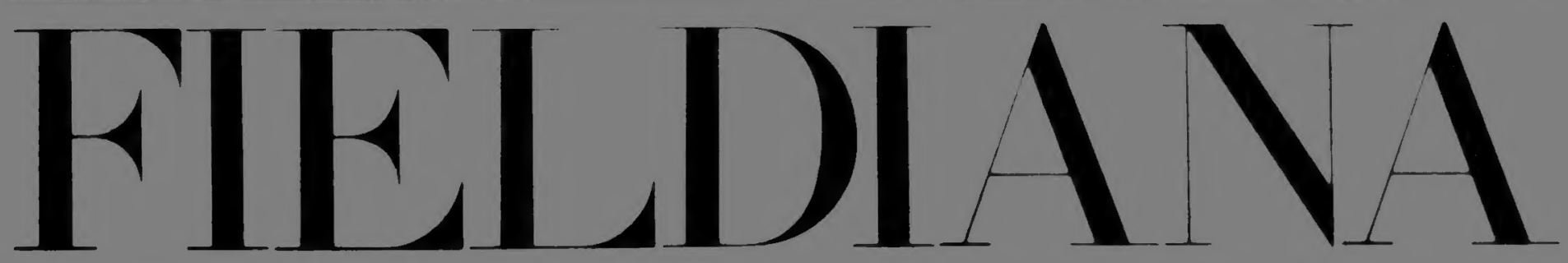

\title{
Zoology
}

NEW SERIES, NO. 114

\section{The Avifauna of Mt. Kitanglad, Bukidnon Province, Mindanao, Philippines}

A. Townsend Peterson

Thomas Brooks

Anita Gamauf

Juan Carlos T. Gonzalez

Neil Aldrin D. Mallari

Guy Dutson

Sarah E. Bush

Dale H. Clayton

Renato Fernandez 
. 


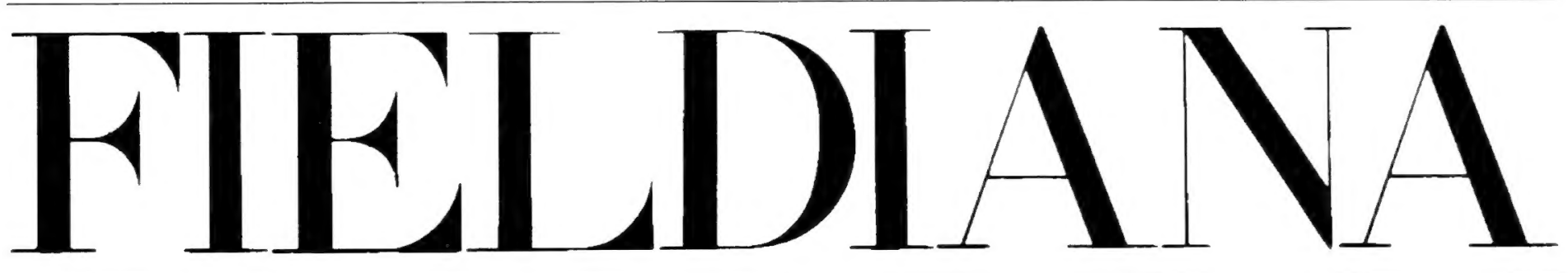

\section{Zoology}

NEW SERIES, NO. 114

\section{The Avifauna of Mt. Kitanglad, Bukidnon Province, Mindanao, Philippines}

\section{A. Townsend Peterson}

The Field Museum, 1400 South Lake Shore Drive Chicago, IL 60605-2496, U.S.A.

Present address:

Natural History Museum

The University of Kansas, Lawrence, KS 66045, U.S.A.

\section{Thomas Brooks}

Center for Applied Biodiversity Science

Conservation International, $1919 \mathrm{M}$ Street NW

Suite 600, Washington, D.C. 20036, U.S.A. and

Department of Environmental Science, University of

Virginia Charlottesville, VA 22904, U.S.A.

Present address:

Center for Applied Biodiversity Science Conservation International, SEARCA, House A

Doña Aurora Street, UPCO

University of the Philippines

Los Baños College, Laguna, 4031, Philippines

\section{Anita Gamauf}

Konrad Lorenz-Institute for Comparative Ethology Austrian Academy of Sciences, Savoyenstraße 1A A-1160 Vienna, Austria

Present address:

Museum of Natural History Vienna

Department of Vertebrate Zoology-Bird Collection

Burgring 7, A-1014 Vienna, Austria

Accepted February 5, 2008

Published June 12, 2008

Publication 1548

\section{Juan Carlos T. Gonzalez}

Animal Biology Division, Institute of Biological Sciences College of Arts and Sciences

University of the Philippines-Los Baños College

Laguna 4031, Philippines

\section{Neil Aldrin D. Mallari}

Environmental and Geographical Sciences Department John Dalton Building, Manchester Metropolitan University, Manchester, U.K.

\section{Guy Dutson}

1 High Way, Broadstone, Dorset BH18 9NB, U.K.

\section{Sarah E. Bush}

Department of Biology. University of Utah, 257 South 1400 East, Salt Lake City, UT 84112, U.S.A.

\section{Dale H. Clayton}

Department of Biology. University of Utah 257 South 1400 East, Salt Lake City, UT 84112 U.S.A.

\section{Renato Fernandez}

Samar Island Biodiversity Project, SSPC-SRSF Complex Barangay Mercedes, Catbalogan, Samar 6700 Philippines 
(C) 2008 Field Museum of Natural History

ISSN 0015-0754

PRINTED IN THE UNITED STATES OF AMERICA 


\section{Table of Contents}

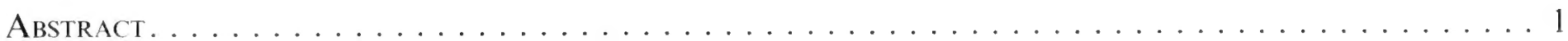

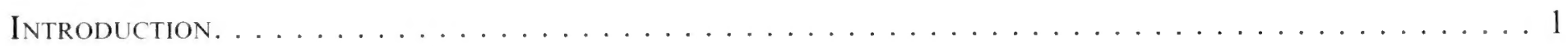

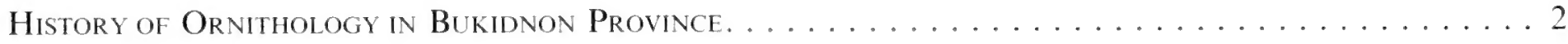

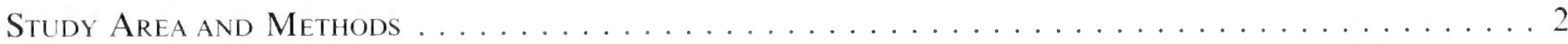

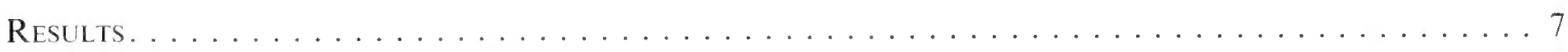

Species Accounts . . . . . . . . . . . . . . . . . . . . . . . . . . . . . . . . . .

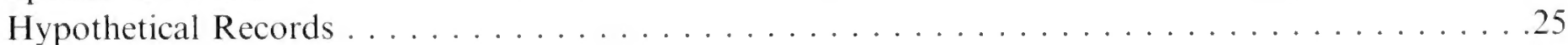

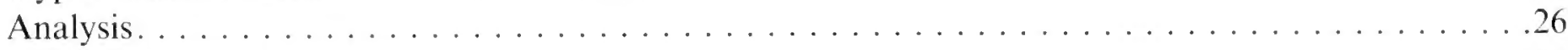

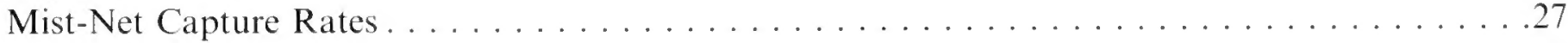

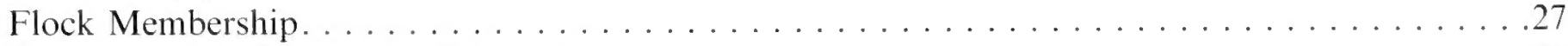

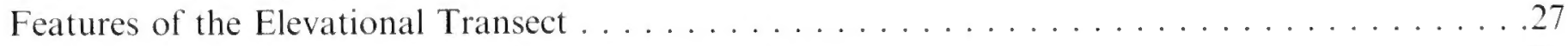

Adequacy of Sampling along the Elevational Transect. . . . . . . . . . . . . . . . . . . . 29

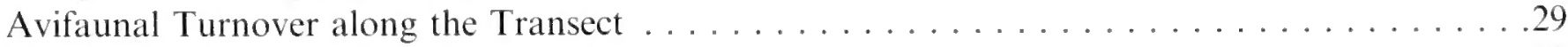

Elevational Overlap of Congeners . . . . . . . . . . . . . . . . . . . . . . . . . . . 29

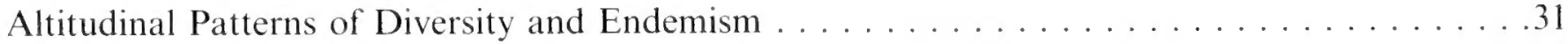

Single-Site Inventory . . . . . . . . . . . . . . . . . . . . . . . . . . . . . . . . . . . . . . . . . . . . . .

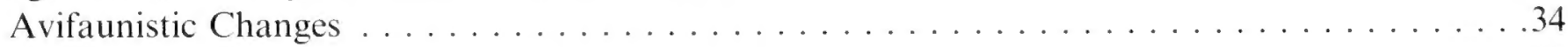

Conservation of Mindanao's Endemic Species. . . . . . . . . . . . . . . . . . . . . . . . . . . . . . . . . . . . . . .

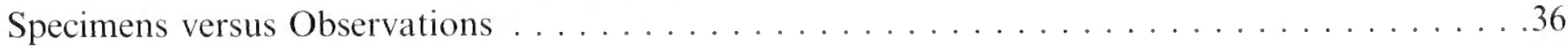

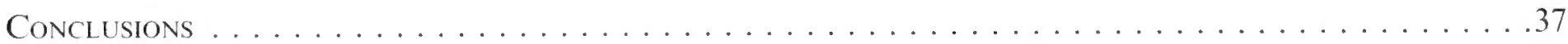

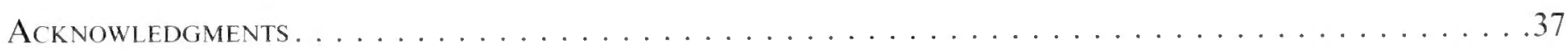

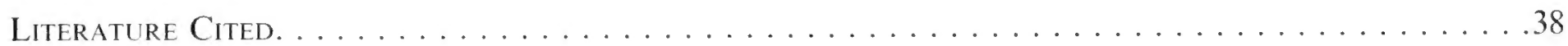

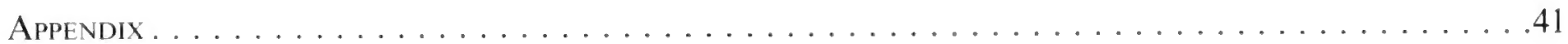




\section{List of Illustrations}

1. Map of Mindanao and the Kitanglad massif $\ldots \ldots \ldots \ldots \ldots \ldots \ldots \ldots \ldots \ldots \ldots \ldots$

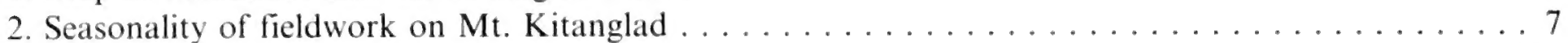

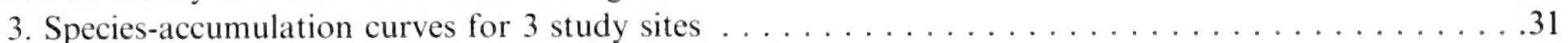

4. Elevational trends in species diversity and endemism . . . . . . . . . . . . . . . . .34

\section{List of Tables}

1. Summary of unpublished bird reports incorporated in this study $\ldots \ldots \ldots \ldots \ldots \ldots \ldots \ldots$

2. Summary of presumed resident bird species known from the Mt. Kitanglad massif $\ldots \ldots \ldots \ldots 10$

3. Summary of information on migratory bird species known from Mt. Kitanglad. . . . . . . . . . 14

4. Summary of number of individuals captured and individuals per 1000 net-hours captured at 2 elevational levels on Mt. Kitanglad by the $1992-1993$ FMnH groups. . . . . . . . . . . . . . . . 28

5. Summary of species observed participating in multispecies flocks at 2 different elevational levels on

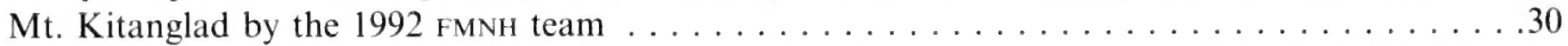

6. Summary of species recorded at 8 elevational stations along the transect covered by the 1992-1993 FMNH groups . . . . . . . . . . . . . . . . . . . . . . 32 


\title{
The Avifauna of Mt. Kitanglad, Bukidnon Province, Mindanao, Philippines
}

\author{
A. Townsend Peterson, ${ }^{1}$ Thomas Brooks, ${ }^{2}$ Anita Gamauf, ${ }^{3}$ \\ Juan Carlos T. Gonzalez, Neil Aldrin D. Mallari, Guy Dutson, \\ Sarah E. Bush, Dale H. Clayton, and Renato Fernandez
}

\begin{abstract}
Intensive inventory work on the Mt. Kitanglad massif of north-central Mindanao, Bukidnon Province, Republic of the Philippines, revealed a diverse avifauna. In all, 198 bird species were detected, of which about 172 were likely breeding in the area, placing the mountain among the most diverse sites that have been surveyed in the country. Three migratory species (Luscinia calliope, Turdus chrysolaus, and Motacilla alba) were detected on the island for the first time by contributors to this summary, and known ranges of several species were extended. The massif holds populations of at least 17 of the 18 species narrowly endemic to Mindanao - more than any other site yet studied-as well as almost half the species endemic to the entire Philippine archipelago. Endemic species are concentrated at higher elevations, whereas migrants and widespread species are more frequent in the lowlands. The Kitanglad massif, however, has been modified severely below about $1200 \mathrm{~m}$, with removal of almost all lowland forest habitats. Records and data accumulated by historical collectors, recent collectors, and recent observers are compared and contrasted to reflect on the biases, strengths, and omissions in each. The importance of the Kitanglad region to effective conservation of biodiversity in the southern Philippines is particularly emphasized.
\end{abstract}

\section{Introduction}

The study of regional patterns of biological diversity is a complex challenge. Patterns of geographic distribution, species limits, variation, and differentiation - alpha-taxonomy - must be studied and defined for each species. Single-site inventories must be conducted to determine local levels of diversity and integrated into a regional view of spatial variation in diversity and endemism (Colwell \& Coddington, 1994). These patterns can then be compared with features of the physical and biological environment to produce a synthetic understanding of determinants of patterns of biological diversity. Obviously, this process is labor intensive and time consuming, but completion of such syntheses of regional patterns of biological diversity is crucial both to conservation action and to many scientific questions regarding the process of biological diversification.

The many islands that make up the Republic of the Philippines have an odd history of ornithological exploration (Dickinson et al., 1991). The earliest collections from the region were the occasional and opportunistic samples that characterize those of much of the Tropics, with a few more comprehensive expeditions by European and American groups (summarized in Dickerson, 1928). In-depth, systematic sampling did not begin until after World War II, when D. $\mathrm{S}$. Rabor initiated a long series of inventories of sites throughout the Philippines (Dickinson et al., 1991). Rabor's collections are immense (perhaps 60,000+ bird specimens, widely dispersed around the world's museums) (Kennedy \& Miranda, 1998) and could constitute a foundation for thorough documentation of 
many aspects of Philippine biological diversity if properly evaluated and interpreted. Recent landmark detailed studies (Goodman \& Gonzales, 1989; Goodman et al., 1995) and country summaries (Dickinson et al., 1991; Collar et al., 1999; Kennedy et al., 2000) have provided synthesis of knowledge accumulated to date.

These studies have become especially relevant because of the crisis of conservation of tropical forests and their biological diversity, with the Philippines ranking among the countries with greatest threat of forest destruction and with the most biological diversity to lose (Brooks et al., 1997; Heaney \& Regalado, 1998; Myers et al., 2000). No longer are such studies of interest only to systematic biologists. Now, each study must be placed in a perspective of faunal composition and levels of endemism because the results should form the basis for decisions regarding the relative importance of different sites for conservation action.

The present contribution thus has 2 chief objectives: (1) We present an inventory of the birds of a single mountain range - the Mt. Kitanglad massif in north-central Mindanaowhich can stand with other such studies as points of reference for interpreting patterns at sites not yet studied, and (2) we compare the results of our studies with those of past workers in the region (Ripley \& Rabor, 1961) both to understand changes that have occurred in the avifauna and to begin to provide a general yardstick to aid in understanding Rabor's surveys elsewhere.

\section{History of Ornithology in Bukidnon Province}

The earliest scientific explorations of Mindanao were in the 1770 s by Sonnerat, in the Zamboanga Peninsula, but the Mindanao highlands were not visited until more than a century later, when Montano climbed Mt. Apo in the 1880s (Dickinson et al., 1991). Many new species endemic to Mt. Apo, Mindanao as a whole, or Greater Mindanao (the group of islands that joined in the Pleistocene to form 1 large island) (Heaney, 1986) were discovered on these early explorations.

The ornithological exploration of northern Mindanao began with work by Everett in the 1870s and Zimmer's visit in 1915 to Mailag and Sumilao in Bukidnon Province (Zimmer, 1918). The Danish Philippine Expedition of 1951-1952 was first to climb high into the Mt. Kitanglad massif (Salomonsen, 1953). D. S. Rabor and R. Gonzales followed with a series of expeditions into the Kitanglad region in the early 1960s that revealed a new species, Erythrura coloria, and the only known Philippine populations of Serinus estherae (Ripley \& Rabor, 1961). Aside from brief visits by Gonzales during studies of Pithecophaga jefferyi (Gonzales, 1968), our own fieldwork, and a 1996 survey by NORDECO and DENR (1998), the region has not seen attention from museumbased ornithologists in recent decades.

Starting in the early 1980s, Kitanglad became a focus of activity for bird-watchers. Numerous organized groups, often guided by T. Fisher and organized through KingBird (B. King) and BirdQuest (S. Harrap, N. Redman, and P. Morris), have visited the mountain, along with increasing numbers of independent bird-watchers. Many of these bird-watchers have kindly provided us with trip reports (Table 1; Lambert, 1993; Hornskov, 1995); others kindly provided information on their observations as well (e.g., G. Anderson, R. Timmins, I. Gardner, N. Bostock, P. Heath, A. Long, M. Heath, S. Hayhow, C. Bell, A. Pierce, M. Fehlow, P. Thompson, J. Gregory, and A. Barnwell). This information, although not substantiated by specimen vouchers, nonetheless serves to enrich the picture of local bird diversity greatly. Overall, although Kitanglad is remote, this rich history of ornithological exploration invites a thorough review and avifaunal analysis.

\section{Study Area and Methods}

The Kitanglad range has an area of about 31,300 ha and is centered at about $8^{\circ} 7.2^{\prime} \mathrm{N}$, $124^{\circ} 54.6^{\prime} \mathrm{E}$, approximately $70 \mathrm{~km}$ southeast of Cagayan de Oro and 20-30 km west of Malaybalay, Bukidnon. It consists of several mountain peaks of 1500-2938-m elevation surrounded by plains at $500-600 \mathrm{~m}$. Much of the lowland forest has been disturbed or cleared through extensive logging and conversion to agriculture; the little that is left is under heavy pressure from shifting agriculture and illegal logging. While forests on several major peaks in the range have burned, either because of lightning or anthropogenic causes (e.g., Mt. Kitanglad Peak, $2899 \mathrm{~m}$ ), important parts of the range (e.g., Mt. DulangDulang, $2938 \mathrm{~m}$ ) still support some primary lowland and extensive primary montane and mossy forests. The importance of the massif in a 
TABle 1. Summary of unpublished bird reports incorporated in this study.

\begin{tabular}{|c|c|c|c|}
\hline & Observers & Year & Title \\
\hline a & Fairbank, R. & 1996 & Philippines Trip: Apr. 1996 \\
\hline b & Gee, B. & 1997 & The Philippines, Winter 1996/97 \\
\hline c & Gonzales, J. C. T., and N. A. D. Mallari & 1993 & $\begin{array}{l}\text { The Transitional Montane-mossy Forest Avifauna } \\
\text { of Mount Kitanglad National Park, Bukidnon, } \\
\text { Mindanao Island, Philippines }\end{array}$ \\
\hline d & Greensmith, A. & 1990 & The Philippines, 4 Mar.-9 Apr. 1990 \\
\hline e & $\begin{array}{l}\text { Heaney, L. R., E. A. Rickart, B. R. } \\
\text { Tabaranza, J. C. T. Gonzales, N. A. D. } \\
\text { Mallari, and A. T. Peterson }\end{array}$ & 1993 & $\begin{array}{l}\text { Survey of Vertebrate Diversity in Kitanglad Nature } \\
\text { Park, Mindanao. Final Report for } 1992 \text { and } \\
\text { 1993. }\end{array}$ \\
\hline $\mathrm{f}$ & Hornbuckle, J. & 1994 & $\begin{array}{l}\text { Birdwatching in the Philippines. Trip Report for } \\
\text { Jan.-Feb. } 1994 \text { and Definitive Guide to Key } \\
\text { Sites }\end{array}$ \\
\hline $\mathrm{g}$ & Ishøi, F., and M. Trasborg & 1999 & Mount Kitanglad. 2/12-10/12 1998 \\
\hline $\mathrm{h}$ & Jakobsen, M. K., and N. S. Andersen & 1999 & $\begin{array}{l}\text { Complete Systematic List of Birds Seen on Mount } \\
\text { Katanglad. Friday } 3 \text { April-Friday } 10 \text { April } 1998 .\end{array}$ \\
\hline $\mathrm{i}$ & Jensen, S., and J. Hornskov & 1992 & The Philippines, Feb. 17-July 51987 \\
\hline $\mathrm{j}$ & Jensen, S., and J. Hornskov & 1993 & The Philippines \\
\hline $\mathrm{k}$ & Jones, L. & 1992 & $\begin{array}{l}\text { Bird List for the Philippines. } 17 \text { February to } 11 \\
\text { March } 1992\end{array}$ \\
\hline 1 & King, B. F. & 1983 & King Bird Tour April/May 1983 \\
\hline $\mathrm{m}$ & Lewis, I. & 1991 & The Philippines: 23rd February-16th March 1991 \\
\hline$n$ & Mark, T. & 1994 & Philippines: Three Sites 15 Sept. to 26 Sept. 1994 \\
\hline o & Simpson, B. & 1995 & $\begin{array}{l}\text { The Philippines (Mindanao, Dinagat, Bohol, Cebu, } \\
\text { Siquijor, Palawan, Olango Island, Ursula Island } \\
\text { and Manila). 14th December ' } 94 \text { to } 9 \text { th February } \\
\text { ' } 95\end{array}$ \\
\hline
\end{tabular}

number of respects is clear: besides its potential for biodiversity conservation, it is a major watershed, with more than 20 rivers flowing from the range in all directions.

ATP, JCTG, NADM, and RF conducted fieldwork and collected specimens at elevations of 500-2250 m on Kitanglad (Fig. 1) in 19921993. In Apr.--May 1992, 3 sites were surveyed in detail (1100-m camp, 1600-m camp, 1800-m ridge) along an elevational transect up the northwest face of Mt. Imbayao (Table 1e). At the base of this transect $(500 \mathrm{~m})$, a local settlement, Camp Roa, was less than 5 years old, creating disturbance along the road from San Vicente, a larger town a few kilometers distant. Disturbed midmontane dipterocarp forest extended from about 700 to $1100 \mathrm{~m}$, followed by primary midmontane forest to approximately $1600 \mathrm{~m}$. Primary montane forest was present up to the transitional montane-mossy forest at the 1800-m peak. In Mar.-Apr. 1993, JCTG, NADM, and RF surveyed 2 higher sites $(1900 \mathrm{~m}$ and $2250 \mathrm{~m})$ on the north face of Mt. Nangkabulos, which were in primary upper montane forest and lower mossy forest, respectively (Table 1c). Details of the 5 intensively studied sites are as follows: 1100-m Camp - Mindanao, Bukidnon Province, Mt. Kitanglad Range, Mt. Imbayao Peak, $15 \mathrm{~km} \mathrm{~S}, 6 \mathrm{~km}$ E Baungon, $1100 \mathrm{~m}$, $8^{\circ} 11^{\prime} \mathrm{N}, 124^{\circ} 44.5^{\prime} \mathrm{E}, 16$ Apr.-1 May 1992.

This site was in primary midmontane forest along a broad ridge with steep sides. Canopy height was $20-25 \mathrm{~m}$, with emergent trees reaching ca. $30 \mathrm{~m}$; tree diameters ranged $25-$ $60 \mathrm{~cm}$. Canopy vines, climbing bamboo, pandans, and epiphytes including orchids, ferns, and moss, were present but not common. A thin layer of leaf litter covered most of the ground. The soil was weathered volcanic ash, with some small stones; a thin layer of humus covered most of the ground, reaching a maximum depth of ca. $5 \mathrm{~cm}$. Fallen logs were common, and large exposed rocks were present along the ridgetop and streams.

1600-m Camp-Mindanao, Bukidnon Province, Mt. Kitanglad Range, Mt. Imbayao Peak, $15 \mathrm{~km} \mathrm{S,} 7 \mathrm{~km}$ E Baungon, $1600 \mathrm{~m}$, $8^{\circ} 10^{\prime} \mathrm{N}, 124^{\circ} 45^{\prime} \mathrm{E}, 28$ Apr.-21 May 1992.

This site was located on a broad ridge in primary montane forest, with gentle slopes on the ridgetop, and $30-60^{\circ}$ slopes on the sides. Emergent trees, including a conifer (Agathis), had an average height of $20 \mathrm{~m}$ with diameters 

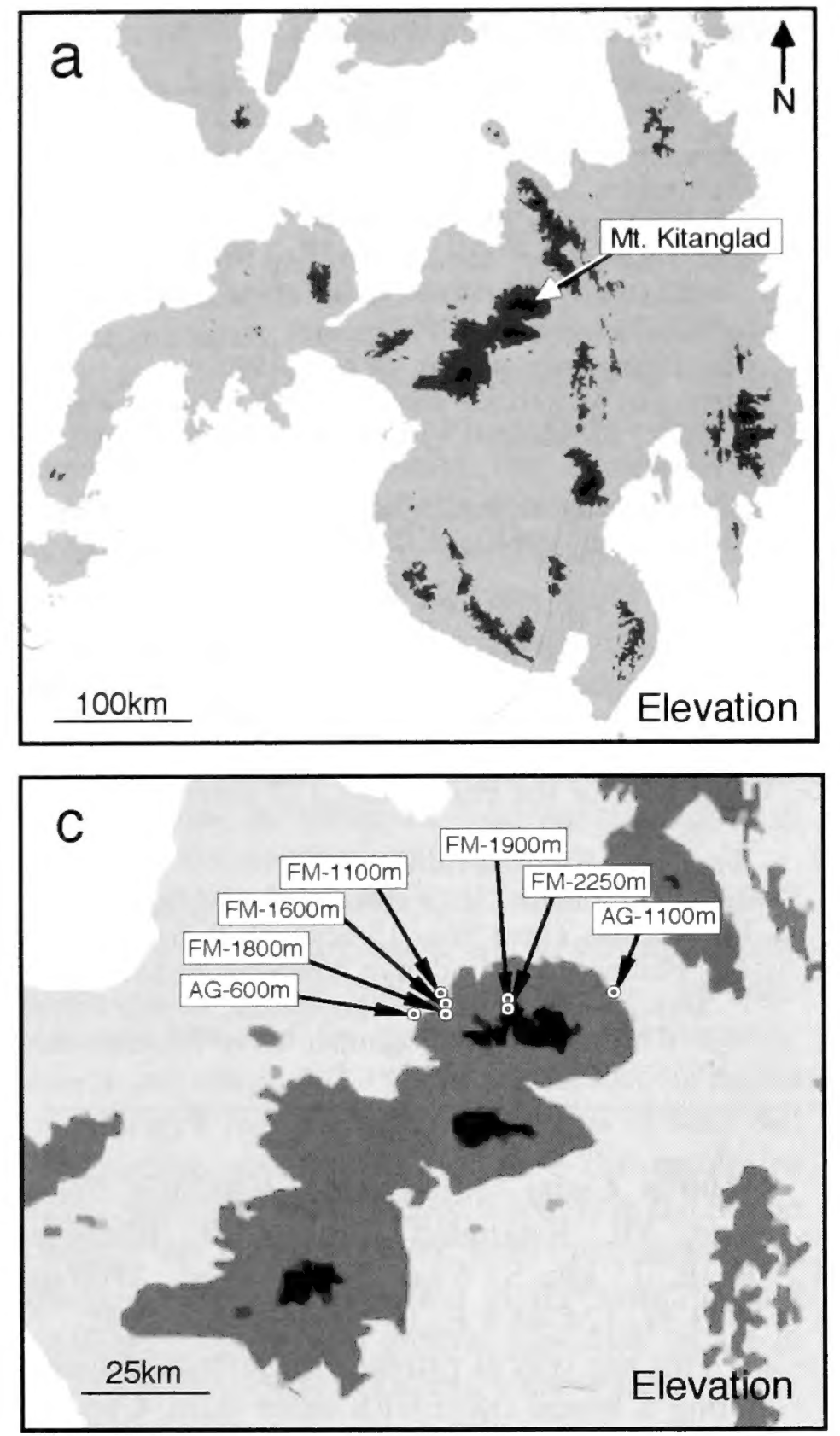

of $30-60 \mathrm{~cm}$. Canopy trees, including some oaks and a Cinnamomum, were about $15 \mathrm{~m}$ tall, with diameters of about $30 \mathrm{~cm}$. Common epiphytes included ferns (including bird's-nest ferns), orchids, and hanging moss. Canopy vines (pandans and rattans) were moderately common. The understory was dominated by saplings, a few tree ferns (Cyathea), erect screw-pine Pandanus, and some Melastoma. Occasional patches of tall "sawgrass" were present. Ficus density was low, but other fruiting trees were fairly common, both in the canopy and in the understory. Density of moss was low to moderate, found on tree trunks, logs, and old trees and some on the ground. The ground surface was covered by $2-6 \mathrm{~cm}$ of leaf litter, on top of a humus layer typically $4-8 \mathrm{~cm}$ deep. Disturbance consisted of a few scattered tree falls.

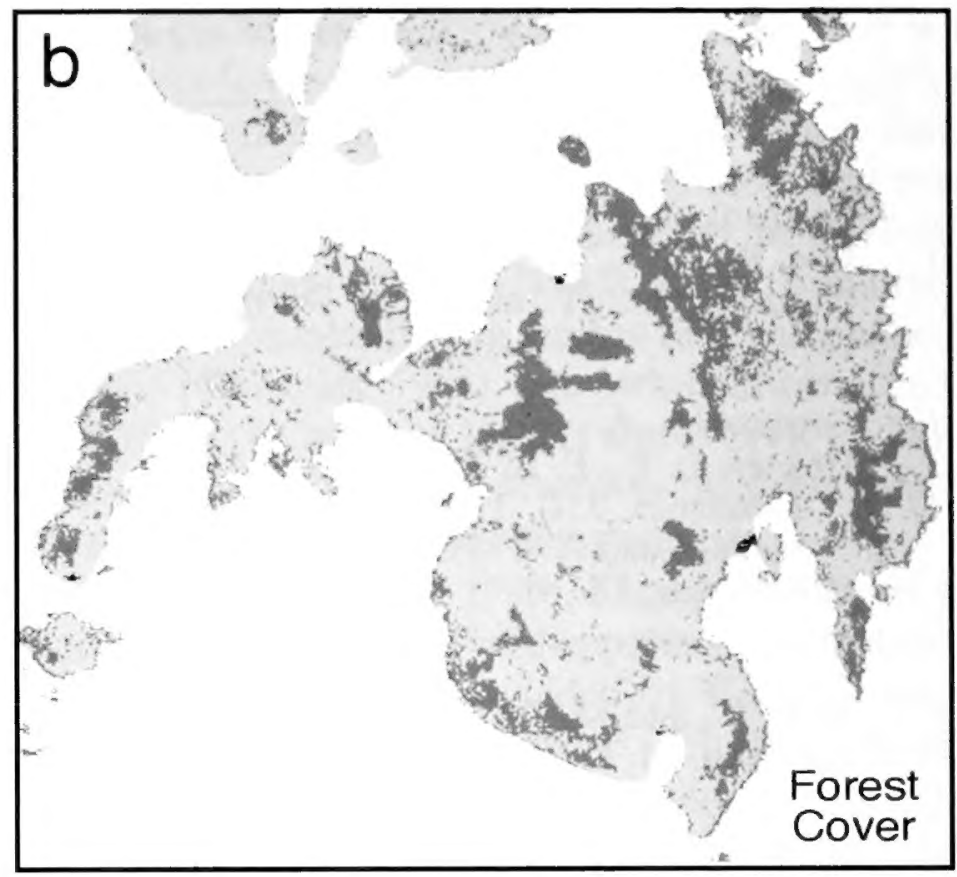

FIG. 1. Map of Mindanao, showing (a) position of highland areas (white is ocean, light gray is $<1000 \mathrm{~m}$, medium gray is $>1000 \mathrm{~m}$ and $<2000 \mathrm{~m}$, black is $>2000 \mathrm{~m}$ ), (b) distribution of native forest on Mindanao (dark gray; U.S. Geological Survey, 2000), and (c) the Kitanglad massif in detail (camps and study sites mentioned in the text are labeled, elevations shaded as in a).

1800-m Ridge - Mindanao, Bukidnon Province, Mt. Kitanglad Range, Mt. Imbayao Peak, $15 \mathrm{~km} \mathrm{~S}, 7 \mathrm{~km}$ E Baungon, $1800 \mathrm{~m}$, $8^{\circ} 9^{\prime} \mathrm{N}, 124^{\circ} 45^{\prime} \mathrm{E}, 7-21$ May 1992.

This site was in primary transitional montane/mossy rain forest, near a small grasscovered peak. The moderately open canopy included trees up to $15 \mathrm{~m}$ tall on the ridge sides and $10 \mathrm{~m}$ on the ridgetops. Undergrowth was heavy, and fallen logs were common. Moss grew on tree trunks, limbs, and twigs from the ground to the canopy, thickly on the trunks and ground but thinner elsewhere. Ferns, orchids, and other plants grew abundantly as epiphytes, and pandan vines were abundant. Leaf litter was profuse, and the humus layer was thick (more than $1 \mathrm{~m}$ in most places) and spongy. A thick root mat on the surface gave the ground a springy resilience. Long strings of 
epiphytes similar to Spanish moss hung from many trees. Fog was quite common.

1900-m Camp-Mindanao, Bukidnon Province, Mt. Kitanglad Range, Mt. Nangkabulos Peak, $16.5 \mathrm{~km} \mathrm{S,} 4 \mathrm{~km}$ E Camp Phillips, $1900 \mathrm{~m}, 8^{\circ} 10.5^{\prime} \mathrm{N}, 124^{\circ} 51^{\prime} \mathrm{E}, \mathrm{Mar}$-Apr. 1993.

This site was in primary upper montane rain forest, in an area characterized by steep slopes and fairly narrow ridgetops. Canopy height was typically $15-20 \mathrm{~m}$, but emergents reached 20-25 m; none of the trees had buttresses. Tree diameters averaged $20-30 \mathrm{~cm}$. Oaks and laurels were common, and a few strangler fig trees were present but no dipterocarps. Ferns and moss were common epiphytes; "Spanish moss" and orchids were less common. Canopy vines (especially pandans) were common. Fallen logs, often rotten, were common. Ground cover consisted of ferns, climbing ferns, small shrubs, wild raspberry, and moss. Leaf litter covered virtually the entire ground surface, usually $1-2 \mathrm{~cm}$ deep, underlain by a layer of moist humus $10-30 \mathrm{~cm}$ deep on top of weathered volcanic ash.

2250-m Camp-Mindanao, Bukidnon Province, Mt. Kitanglad Range, Mt. Nangkabulos Peak, $15.5 \mathrm{~km} \mathrm{S,} 4 \mathrm{~km}$ E Camp Phillips, $2250 \mathrm{~m}, 8^{\circ} 9.5^{\prime} \mathrm{N}, 124^{\circ} 51^{\prime} \mathrm{E}, \mathrm{Mar}-\mathrm{Apr} .1993$.

This site was located in primary lower mossy rain forest characterized by steep slopes (typically $20-45^{\circ}$ ). The incomplete canopy averaged $7-10 \mathrm{~m}$ high, with emergents reaching $12-15 \mathrm{~m}$ on ridgetops and $14-18 \mathrm{~m}$ on ridge sides. None of the trees had buttresses; DBH averaged $20-50 \mathrm{~cm}$, but a few reached $110 \mathrm{~cm}$. The trees largest in diameter were gymnosperms of at least 2 species, including Podocarpus sp. Moss, "Spanish moss," ferns, and orchids were common epiphytes; canopy vines were nearly absent. Fallen logs, many quite rotten, were common. Understory and ground cover plants were abundant, including Rhododendron as a common element, fruitbearing shrubs, saplings, ferns, and climbing ferns. Moss was common on and near the ground, $1-3 \mathrm{~cm}$ thick on trunks and fallen logs, but was scarce more than $2 \mathrm{~m}$ above the ground. Leaf litter covered virtually the entire ground to a depth of 5-20 mm, lying on top of a layer of humus $10-50 \mathrm{~cm}$ in thickness that lay over weathered volcanic ash with scattered large rocks (up to $4 \mathrm{~m}$ in diameter) that protruded through the humus and leaves.
The Field Museum inventories were carried out during 2 separate trips to the mountain massif. At each site, lines of 3-15 mist nets ( $12 \mathrm{~m}$ long, 3-cm mesh, set $0-2 \mathrm{~m}$ above ground) were set crossing each major microhabitat type (ridgetops, valleys, streams); these lines were checked 3-8 times daily; birds captured were identified to species and either marked (to prevent double counting in estimates of abundance) and released or preserved as voucher specimens. A total of 174 net-days was accumulated on the 1100-1200$m$ ridges, 297 on the 1700 - $m$ ridge, 68 on the 1800 $\mathrm{m}$ ridge, 66 at $1900 \mathrm{~m}$, and 57 at $2250 \mathrm{~m}$. These nets were set to maximize bat as well as bird captures (e.g., with nets in more open areas, ridgetops, and so on than would have been used otherwise). Additional avian specimens were collected from snap-traps during intensive mammal trapping on the 1100-1300-m, 1700-1850-m, and 1900-2250- $\mathrm{m}$ ridges; many terrestrial, invertebrate-eating species were collected, especially at higher elevations, where traps were baited principally with earthworms. Additional records were accumulated by extensive daily observations, by surveys with tape recorders later analyzed with reference to work elsewhere in the Philippines, and by limited hunting with an air rifle. Specimens were prepared as either study skins, skeletons, or skin/skeleton preparations or were preserved whole in formalin and were deposited at the Philippine National Museum and the Field Museum of Natural History (Appendix). Frozen tissues were preserved from all specimens.

The 700-1850-m and 1900-2250-m portions of the Field Museum transect were carried out in different sectors of the mountain range. The lower portion was done on the northwest slopes (climbing up from Cagayan de Oro along the Baungon River to the settlements of San Vicente and Camp Roa), where lowland forest was most extensive. That extreme of the mountain range, however, has its peak at 1850-1900 m, so additional studies had to be carried out farther east, on the north face, where higher-elevation forest was available. However, given nearasymptotic species discovery curves and a high degree of coincidence between the results of the 2 portions of the transect in terms of species' elevational limits, we feel confident that the 2 field seasons can indeed be combined to provide a picture of altitudinal changes along the slopes of Mt. Kitanglad.

SEB and DHC worked at several sites (particularly at $10.7 \mathrm{~km} \mathrm{S,} 2.9 \mathrm{~km} \mathrm{~W} \mathrm{Sumilao,}$ 
$8^{\circ} 11^{\prime} 20^{\prime \prime} \mathrm{N}, 124^{\prime} 55^{\prime} 20^{\prime \prime} \mathrm{E}$ ) on the massif on 25 Apr.-4 May 1999 in the course of studies of ectoparasite faunas associated with Philippine birds (e.g., Clayton \& Johnson, 2000); focus was on collection of specimens (particularly of swiftlets) for ongoing ectoparasite studies, resulting in 123 net-days (at $1440-1525 \mathrm{~m}$ ), and 106 specimens of 31 species. Nets were set principally for birds, as contrasted with some of the "bat" nets in the Field Museum work, and no specimens were obtained using firearms.

In the course of studies of Philippine raptors (Gamauf et al., 1998a,b), AG and colleagues conducted fieldwork in 2 areas on Kitanglad in 1993-1994. The northeast foothills (Dalwangan site) were visited 3 times (13-21 Mar. 1993, 2-23 Jan. 1994, and 30 Mar.-11 Apr. 1994, a total of 43 days), and the northwest flank (Kalawaig site) of the massif was visited twice (25-30 Mar. 1993 and 17-29 Apr. 1994, a total of 19 days). The 2 study sites were as follows:

600-m Camp--Mindanao, Bukidnon Province, northwest slope Mt. Kitanglad Range, up the Kalawaig River and its surroundings; study area covers about $45 \mathrm{~km}^{2}, 4.5 \mathrm{~km}$ eastsoutheast of San Antonio (440 m), $1.5 \mathrm{~km} \mathrm{E}$ Lantud (700 m), $8^{\circ} 9^{\prime} \mathrm{N}, 124^{\circ} 42^{\prime} \mathrm{E}, 25-30$ Mar. 1993 and 17-29 Apr. 1994.

The group worked at elevations between $600 \mathrm{~m}$ and $1000 \mathrm{~m}$, occasionally up to $1200 \mathrm{~m}$. The broad, gently inclined slopes were mainly covered by disturbed lowland dipterocarp forest (average canopy height ca. $25 \mathrm{~m}$, with emergents occasionally reaching $35 \mathrm{~m}$; tree diameters ranged from 20 to $40 \mathrm{~cm}$; dense understory). The area was selectively logged in the early 1980 s. The residual forest line was at an elevation of ca. $550 \mathrm{~m}$ and the primary forest line at ca. $900-1100 \mathrm{~m}$ on the broad slopes but as low as $650 \mathrm{~m}$ in the narrow Kalawaig River valley. The indigenous people practice agriculture, which is relatively protective of the forest, with cultivated areas divided into 5-7 portions of equal size. Only 1 of these portions is cultivated each year; the other areas are left fallow to regenerate successively. In this way, each portion is used at 5-7-year intervals for a variety of crops (e.g., vegetables, bananas). Older trees are left standing at the edge of these areas, often providing birds with attractive "fruit trees." This agricultural strategy is the reason why forest fragments are still found at lower elevations. Human disturbance was heaviest in the vicinity of settlements and became lighter as elevation increased.

The main camp was located on the Kalawaig River; however, excursions were made a great distance upriver, to the Tagiti River in the northeast and beyond the Tulohan River in the south. The valleys become increasingly narrow upriver, and valleys are deep and slopes very steep toward the peak of $\mathrm{Mt}$. Imbayao. In the rivers themselves, large exposed rocks are common. The slopes are not generally suited for cultivation, and timber exploitation is very difficult, permitting the persistence of primary forest at low elevations. At the camp, the valley was $>1 \mathrm{~km}$ wide and ca. $300 \mathrm{~m}$ deep at the slopes' shoulders; at the mouth of the Andalawi River, the Kalawaig valley is less than $700 \mathrm{~m}$ wide.

1100-m Camp (Philippine Eagle Conservation Project lodgehouse and areas to the west and southwest, at higher elevations)-Mindanao, Bukidnon Province, northeast slope $\mathrm{Mt}$. Kitanglad Range, ca. $9 \mathrm{~km}$ west of Dalwangan, size of study area about $38 \mathrm{~km}^{2}, 1100 \mathrm{~m}$, $8^{\circ} 11^{\prime} \mathrm{N}, 125^{\circ} 1^{\prime} \mathrm{E}, 13-21$ Mar. 1993, 2-23 Jan. and 30 Mar.-11 Apr. 1994.

This site is in a gently sloping, wide, and relatively dry area between river valleys. The forest was clear-cut or heavily reduced in the early 1970s, mainly for planting coffee. Over the years, the forest boundary has been pushed farther upslope by intensive agriculture by small farmers in the area. Several degraded wooded patches still exist between the cultivated fields and fallow areas. Only along the small rivers (Lalawan River, Sawaga River) did closed forest descend to lower elevations (ca. $900 \mathrm{~m}$ ).

The agricultural areas (potatoes, sweet potatoes, vegetables), cultivated primarily by the Ifugao people (recent immigrants from Luzon), reach up to ca. $1400 \mathrm{~m}$, even within the protected natural park area. Some cultivated areas (kaingins) were even close to the mossy forest line (ca. $1700 \mathrm{~m}$, on slopes of $\mathrm{Mt}$. Tuminungan). Even the narrow floor of the Lalawan River valley was seeing increased cultivation. This valley was ca. $400 \mathrm{~m}$ wide at the shoulders and had steep slopes. The gently inclined slope south of the Lalawan River toward Mt. Dulang-Dulang was selectively 
logged in 1974-1975, but the "Twin Peaks" of Mt. Dulang-Dulang were completely covered with primary forest. Upriver toward Kitanglad, broad forested areas burned because of lightning years ago; they were covered with high grass and charred remains of trees. Work was concentrated at $1000-1600 \mathrm{~m}$, occasionally up to $2100 \mathrm{~m}$.

TB and GD visited the Dalwangan area on 3-6 Oct. 1991, making extensive opportunistic observations up to $1600 \mathrm{~m}$; other information was kindly provided by visiting birdwatchers.

Finally, we summarize records from historical natural history museum collections (abbreviations provided when specific specimens are cited in the text): Academy of Natural Sciences of Philadelphia (ANSP), Bell Museum of Natural History, California Academy of Sciences, Delaware Museum of Natural History (DMNH), Field Museum of Natural History (FMNH), Florida State Museum (FSM), Los Angeles County Museum of Natural History, Museum of Vertebrate Zoology, Philippine National Museum (PNM), Silliman University Natural Science Museum (SUNSM), University of Kansas Natural History Museum (KUNHM), Western Foundation of Vertebrate Zoology, Yale Peabody Museum (YPM), Naturmuseum Senkenberg (SMF), and Zoological Museum of the University of Copenhagen (ZMUC). The seasonal distribution of collecting and birding trips reviewed here is given in Figure 2.

When recent survey data were sufficiently extensive, we evaluated the completeness of our inventories using quantitative methodologies (Soberón \& Llorente, 1993; Peterson \& Slade, 1998). These approaches consist of extrapolation from known fauna sizes to predicted fauna sizes and then use of both figures to arrive at estimates of percent completeness of inventory data. In particular, we used the bootstrapped Chao equation approach (Peterson \& Slade, 1998), in which species richness is predicted as

$$
S_{\exp }=S_{o b s}+\frac{a^{2}}{2 b}
$$

where $S_{o b s}$ is the number of species actually observed in the study, $a$ is the number of species observed in just 1 sampling period (here, a day of sampling), and $b$ is the number of species observed in exactly 2 sampling

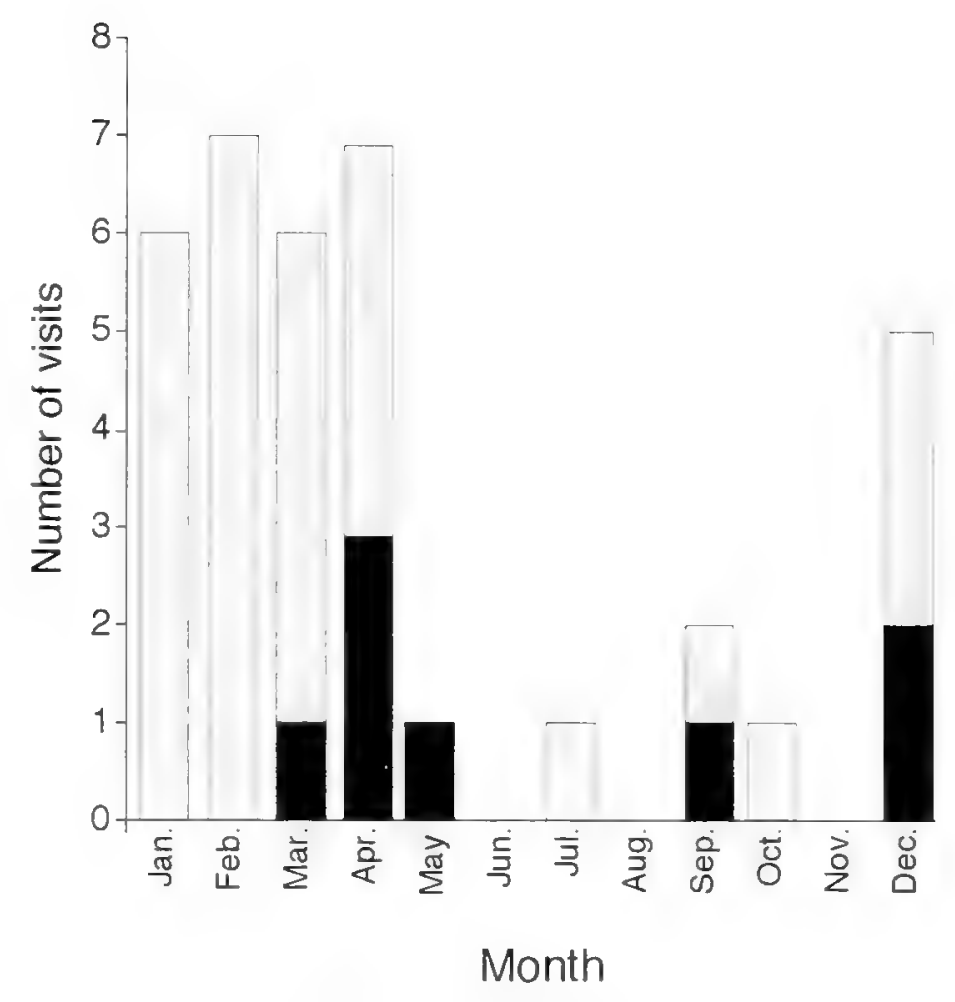

Fig. 2. Seasonality of fieldwork on Mt. Kitanglad, including our own. Collecting expeditions are shaded black (Salomonsen, 1953; Ripley \& Rabor. 1961: NORDECO and DENR. 1998, table $1 \mathrm{c}$ and e): visits by other ornithologists (without voucher specimens) are gray. Given that Kitanglad has strongly seasonal rainfall, the concentration of visits in the relatively dry months of December-April is clear.

periods. $S_{\text {obs }} / S_{\text {exp }}$, then, can be used as a measure of the completeness of the actual inventory of species (Peterson \& Slade, 1998).

\section{Results}

\section{Species Accounts}

In this section, we present in-depth information about selected species that have been documented on Mt. Kitanglad that are of special interest either from an ornithological perspective or in terms of conservation. We discuss species falling into 1 or more of 5 categories: (1) species classed as globally threatened or near threatened (BirdLife International, 2004), (2) species not previously documented from the Kitanglad region, (3) species that have apparently declined on Kitanglad or across broader regions, (4) species for which we noted unusual ecological or behavioral characteristics, or (5) species for which current information updates previous understanding of status on Kitanglad. Nomenclature and systematic order follow Kennedy et 
al. (2000), with exceptions dictated by more recent work indicated by a "†." It should be noted that species accounts are provided only for species of special interest (see above) - the complete list of species known from the Kitanglad massif is summarized in Table 2 (residents) and Table 3 (migrants).

\section{Anas luzonica Philippine Duck VULNERABLE}

Sighted flying over the northeast flank of the mountain in 1994 by P. Heath (F. Verbelen, in litt., 1998). This taxon was recently up-listed from near threatened (Collar et al., 1994) to vulnerable (Collar et al., 1999), although Kitanglad clearly would not constitute a priority site for its conservation.

\section{Pernis celebensis Barred Honeybuzzard}

Although only a single specimen of this species exists from Kitanglad (SMF 47343), numerous recent sightings from the northeast flank also document its presence at the site: an adult seen by N. Redman on 7 Mar. 1990 (Gretton, 1990), 1-2 daily on 12-14 Mar. 1990 (Table 1d), 1 on 6 Oct. 1991 [TB, GD], several on 28-29 Feb. 1992 (Table $1 \mathrm{k}$ ), regular sightings [AG] of 2 pairs between $1180 \mathrm{~m}$ and $1550 \mathrm{~m}$ in 1993-1994 (Gamauf \& Preleuthner, 1998), 2 on 2 Apr. 1996 (Table 1a), and 2 at $1250 \mathrm{~m}$ on 6 Dec. 1998 (Table $1 \mathrm{~g}$ ). The only sightings away from Dalwangan are of 3-4 pairs regularly seen at 600 $800 \mathrm{~m}$ on the northwest flank [AG] in 1993-1994 (Gamauf \& Preleuthner, 1998).

\section{Civcus spilonotus Eastern Marsh-harrier}

Only 1 specimen exists of this species from Mindanao (Dickinson et al., 1991), but an adult male was observed hunting over short grass (ca. $50 \mathrm{~cm}$ tall), and apparently displaying over tall grass on 13-19 Mar. 1993 at $930-1030 \mathrm{~m}$ on the Dalwangan slope [AG], even though this species does not breed in the Philippines (Dickinson et al., 1991). The only other Kitanglad sighting is of 1 seen on 26 Feb. 1991 (Table 1m).

\section{Pithecophaga jefferyi Philippine Eagle \\ CRITICALLY ENDANGERED}

Although Kitanglad is the best-known locality for $P$. jefferyi, its status there is difficult to interpret, as most records derive from a single nest site in the Lalawan Valley, above Dalwangan on the northeast flank. The first records were from 1500 to $1800 \mathrm{~m}$ at Malaybalay $\left(8^{\circ} 09^{\prime} \mathrm{N}\right.$, $125^{\circ} 5^{\prime} \mathrm{E}$ ) in 1959-1964 (Rabor, 1965, 1968, 1971; Gonzales, 1968), with other records (including 4 shot) through the 1960s (Gonzales, 1971). Nesting was recorded at Kinubalan $\left(8^{\circ} 13^{\prime} \mathrm{N}\right.$, $\left.124^{\circ} 59^{\prime} \mathrm{E}\right)$ in 1984 and at Dalwangan $\left(8^{\circ} 14^{\prime} \mathrm{N}\right.$, $125^{\circ} 2^{\prime} \mathrm{E}$ ) in 1982-1993 (Krupa, 1985). This pair is almost certainly the same as that recorded nesting on 30 Dec. 1989 at $1350 \mathrm{~m}$ (Lambert, 1993), 12-16 Mar. 1990 (Table 1d), and Feb.Mar. 1993 (I. Gardner, in litt., 1993, AG). It was also reported that young were visible in the nest in Nov.-Apr. 1990-1991 and 1992-1993 (Table 1f) and that the pair laid a single egg on 28 Nov. 1992 [AG].

It was reported to AG that this pair's eyrie has been pushed farther up the valley (ca. $3 \mathrm{~km}$ in the last $20 \mathrm{yr}$ ), presumably because of increasing habitat disturbance: disturbed areas (kaingin) reached to within $200 \mathrm{~m}$ of the eyrie by 1992 . Apparently, the eyrie was at ca. $1100 \mathrm{~m}$ in the late $1970 \mathrm{~s}$, at ca. $1200 \mathrm{~m}$ by the mid-1980s, and at $1330 \mathrm{~m}$ by $1993-1994$ [AG]. Sight records of individuals presumably from the Dalwangan pair include adults frequently observed hunting at 1150-1700 $\mathrm{m}$, occasionally up to $7 \mathrm{~km}$ from the nest, in 1993 [AG]. Further records are from 1987-1998 (Table 1a, b, f-h, j, k, m-o) and R. J. Timmins (pers. comm., 1992).

Away from Dalwangan, Krupa (1985) reported a bird captured at Libona $\left(8^{\circ} 20^{\prime} \mathrm{N}, 124^{\circ} 44^{\prime} \mathrm{E}\right)$ on the north flank in $1982 ; 1$ recorded soaring over primary forest at $800 \mathrm{~m}$ on the northwest flank $\left(8^{\circ} 9^{\prime} \mathrm{N}, 124^{\circ} 42^{\prime} \mathrm{E}\right)$ on 28 Mar. 1994 [AG]; several further reports are available from the Philippine Eagle Working Group (Collar et al., 1999). These records, from up to $30 \mathrm{~km}$ from Dalwangan, suggest that at least a few other pairs of eagles persist on Kitanglad, especially given that the species' home range is now suspected to be smaller than traditionally assumed (Collar, 1997a,b). Nevertheless, increasing human pressure on Kitanglad's forest, pushing the eagles into what may be suboptimal higher elevation habitat, surely indicates that the species is under extreme threat here, as elsewhere in its range.

\section{Spizaetus cirrhatus Changeable Hawk-eagle}

This species is known on Mindanao from a single specimen (Dickinson et al., 1991) but has been sighted several times on Kitanglad. Hornskov (1995) sighted a pale individual, probably an immature, on 14 Mar. 1987. AG obtained breeding records in Apr. 1994 at 600-1120 m on the northwest slope, as well as observations of a first-year individual seen hunting over fragmented 
forest, and at least 3 pairs observed regularly engaging in territorial displays (Gamauf et al., 1998a).

\section{Spizaetus philippensis Philippine Hawk-eagle VULNERABLE}

Although Dickinson et al. (1991) reported only 1 specimen from Mindanao, AG has traced 3 further skins from other sites on the island. At $1100-1650 \mathrm{~m}$ on the northeast slopes of Kitanglad, 3 pairs and 1 independent juvenile were sighted over $38 \mathrm{~km}^{2}$ in 1993-1994 [AG]. On the northwest slopes, sightings include individuals seen by TB and GD in Sep. 1991 (Evans et al., 1993), by R. J. Timmins (pers. comm., 1992) in Jan. 1992, on 27-29 Feb. 1992 (Table 1k), Feb. 1994 (Table 1f), Apr. 1998 (Table 1h), pairs in Sep. 1994 (Table 1n) and Dec. 1996 (Table 1b), and up to 3 on 2-12 Dec. 1998 at 1250-1520 m (Table 1g). Collar et al. (1999) noted sightings from D. Cedeno, G. Balaquit, and C. Gayamara in Nov. 1994 and from P. Morris of a juvenile in Jan. 1994. Southern populations of the species have recently been recognized as distinct subspecifically (Preleuthner \& Gamauf, 1998), and separation of the species from $S$. cirrhatus in the field has been discussed (Gamauf et al., 1998a).

\section{Falco tinnunculus Eurasian Kestrel}

The only previous Mindanao record of this species was a sight record by Mearns on 31 Mar. (Dickinson et al., 1991). One was seen on 19 Mar. 1993 at $880-1000 \mathrm{~m}$ over pastureland on the northeast slope of Kitanglad [AG].

\section{Falco severus Oriental Hobby}

This species is known from Kitanglad from only 2 specimens, a female collected on 3 May and a male on 22 Dec. 1960 (YPM 61572, 62268) (Ripley \& Rabor, 1961). The absence of recent records is intriguing.

\section{Falco peregrinus Peregrine Falcon}

Although large, dark birds of the race $F . p$. ernesti breed on steep cliffs at a few sites in the Philippines, the only previously suspected breeding locality on Mindanao is Mt. Apo (Dickinson et al., 1991). On Kitanglad, adult peregrines were seen on 3 Apr. $1994(1180-1250 \mathrm{~m})$ on the northeast slope and on 28 Mar. 1993 (640 m) and 23 Apr. $1994(600-700 \mathrm{~m})$ on the northwest slope $[A G]$. In addition, sightings exist for an adult on 11 Jan. 1995 (Table 1o), a "rather pale grey" bird in early Apr. 1996 (Table 1a), and 1 on 3 Dec. and 2 on 5 Dec. 1999 at $1520 \mathrm{~m}$
(Table $1 \mathrm{~g}$ ). We assume that these individuals were wintering, although the possibility remains that a breeding population exists high on Kitanglad.

\section{Gallus gallus Red Junglefowl}

This species may have been introduced into the Philippines (Parkes, 1962), and Philippine populations have apparently been contaminated secondarily by crossing with domestic chickens (Peterson \& Brisbin, 1998). Records from the mountain include 3 specimens from the 1960s (DMNH 2989, YPM 61573, 62269) (Ripley \& Rabor, 1961), and birds seen and heard fairly commonly in 1993-1994 at $630-950 \mathrm{~m}$ on the northwest slope $[\mathrm{AG}]$. On the west slope, it was detected only uncommonly at $1100-1300 \mathrm{~m}$, while at $1500 \mathrm{~m}$ it was heard calling almost every morning in Apr.-May 1992 [ATP, RF]; junglefowl were also sighted at $1900-2250 \mathrm{~m}$ on Mt. Nangkabulos in Mar.-Apr. 1993 [JCTG, NADM]. On the northeast flank, sightings include a male on 15 Mar. 1987 (Table 1j), individuals heard on 14-15 Mar. 1990 (Table 1d), several individuals seen at $1390 \mathrm{~m}$ in secondary forest on 18 Mar. 1993 and at $1800 \mathrm{~m}$ in mossy forest on the east flank of $\mathrm{Mt}$. Tuminungan on 9 Apr. 1994 [AG], a male "sitting c $20 \mathrm{ft}$ up a tree noisily feeding ... 1 mile or so away from the nearest houses" on $17 \mathrm{Dec}$. 1994 (Table lo), and a sighting "in remote forest" in Apr. 1996 (Table la). Hence, a considerable buffer of uninhabited forest separates Kitanglad junglefowl populations from human influence, suggesting that lower-elevation populations have been hunted out or that this species avoids human presence.

\section{Turnix sylvatica Small Buttonquail}

The single Kitanglad report of T. sylvatica, the only Turnix known from Mindanao (Dickinson et al., 1991), is from grasslands on Kitanglad on 11 Mar. 1990 (Table 1d). A buttonquail not specifically identified but presumably pertaining to this species was seen at $520 \mathrm{~m}$ on the northwest slope on 29 Apr. 1994 [AG].

\section{Gallinago megala Swinhoe's Snipe}

Although a common winter visitor to the Philippines (Dickinson et al., 1991), the difficulties of separating this species from Pintail Snipe G. stenura and Common Snipe G. gallinago (Carey \& Olsson, 1995) suggest that records should require specimen vouchers for confirmation. One specimen (SMF 47128) documents this 
TABLE 2. Summary of presumed resident bird species known from the Mt. Kitanglad massif. Species taxonomy follows Dickinson et al. (1991), except as indicated by recent taxonomic treatments. Conservation status from Collar et al. (1999), restricted-range status is from Stattersfield et al. (1998), recent records (since 1983) as observed and summarized herein, recent specimens (1992 1993, 1999) deposited at PNM and FMN, and historical specimens (pre-1970; Acknowledgments). For endemism, P = endemic to the Philippines, GM = endemic to Greater Mindanao, and $\mathrm{M}=$ endemic to Mindanao. For conservation status, $\mathrm{CR}=$ critical, $\mathrm{VU}=$ vulnerable, $\mathrm{NT}=$ near threatened, and DD = data deficient. $\uparrow$ denotes exceptions dictated by more recent work.

\section{Taxon}

Endemism tion status

Restricted Recent Recent Historical
range records specimens specimens

Family Ardeidae

Butorides striatus Little Heron

Dupetor flavicollis Black Bittern

Family Anatidae

Anas luzonica Philippine Duck

Family Accipitridae

Accipiter trivirganus Crested Goshawk

A. virgatus Besra

Elanus caeruleus Black-shouldered Kite

Haliastur indus Brahminy Kite

Hieraaetus kienerii Rufous-bellied Eagle

Pernis celebensis Barred Honeybuzzard

$P$. ptiloryncus Oriental Honeybuzzard

Pithecophaga jefferyi Philippine Eagle

Spilornis holospilus Philippine Serpent-Eagle $\dagger$

Spizaetus cirrhatus Changeable Hawk-eagle

S. philippensis Philippine Hawk-eagle

Family Falconidae

Falco severus Oriental Hobby

Microhierax erythrogenys Philippine Falconet

Family Phasianidae

Gallus gallus Red Junglefowl

Family Turnicidae

Turnix sylvatica Small Buttonquail

Family Rallidae

Amaurornis olivacea Plain Bush-hen

A. phoenicurus White-breasted Waterhen

Gallirallus philippensis Buff-banded Rail

G. torquatus Barred Rail

Family Scolopacidae

Scolopax bukidnonensis Bukidnon Woodcock $\uparrow$

Family Columbidae

Chalcophaps indica Common Emerald-dove

Columba vitiensis Metallic Pigeon

Ducula carola Spotted Imperial-pigeon

$D$. poliocephala Pink-bellied Imperial-pigeon

Geopelia striata Zebra Dove

Macropygia tenuirostris Philippine Cuckoo-dove Phapitreron amethystina Amethyst Brown-dove

$P$. brunneiceps Mindanao Brown-dove $\dagger$

$P$. leucotis White-eared Brown-dove

Ptilinopus occipitalis Yellow-breasted Fruit-dove

Streptopelia bitorquata Island Turtle-dove

S. chinensis Spotted Dove

Family Psittacidae

Bolbopsittacus lumulatus Guiabero

Loriculus philippensis Colasisi

Prioniturus discurus Blue-crowned Racquet-tail

$P$. waterstradti Mindanao Racquet-tail $\dagger$

Trichoglossus johnstoniae Mindanao Lorikeet

Family Cuculidae

Cacomantis sepulcralis Indonesian Cuckoo $\dagger$

Centropus bengalensis Lesser Coucal

C. melanops Black-faced Coucal

\begin{tabular}{|c|c|c|}
\hline 一 & - & - \\
\hline- & - & - \\
\hline$P$ & VU & - \\
\hline - & - & - \\
\hline - & - & - \\
\hline - & - & - \\
\hline - & - & - \\
\hline - & - & - \\
\hline - & - & - \\
\hline - & - & - \\
\hline $\mathrm{P}$ & CR & \\
\hline $\mathrm{P}$ & - & - \\
\hline- & - & - \\
\hline $\mathrm{P}$ & VU & $\ldots$ \\
\hline- & - & - \\
\hline $\mathrm{P}$ & - & - \\
\hline - & - & - \\
\hline 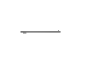 & - & - \\
\hline $\mathrm{P}$ & - & - \\
\hline- & - & - \\
\hline- & - & - \\
\hline- & - & - \\
\hline $\mathrm{P}$ & - & X \\
\hline- & - & - \\
\hline 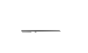 & - & - \\
\hline $\mathrm{P}$ & VU & - \\
\hline $\mathrm{P}$ & NT & - \\
\hline 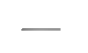 & - & $\cdots$ \\
\hline & - & - \\
\hline $\mathrm{P}$ & - & - \\
\hline $\mathrm{GM}$ & VU & $X$ \\
\hline $\mathrm{P}$ & - & - \\
\hline$P$ & - & - \\
\hline- & - & - \\
\hline - & - & - \\
\hline$P$ & - & - \\
\hline $\mathrm{P}$ & - & - \\
\hline $\mathrm{P}$ & - & - \\
\hline $\mathrm{M}$ & NT & $X$ \\
\hline $\mathrm{M}$ & NT & $X$ \\
\hline- & - & - \\
\hline & - & - \\
\hline & - & - \\
\hline
\end{tabular}

\section{$X$
$X$}

$\mathrm{X}$

$\begin{array}{lll}\mathrm{X} & - & - \\ \mathrm{X} & - & \mathrm{X} \\ \mathrm{X} & \mathrm{X} & - \\ \mathrm{X} & - & - \\ \mathrm{X} & - & \mathrm{X} \\ \mathrm{X} & - & - \\ \mathrm{X} & - & \mathrm{X} \\ \mathrm{X} & - & - \\ \mathrm{X} & - & - \\ \mathrm{X} & - & \mathrm{X} \\ \mathrm{X} & - & - \\ \mathrm{X} & - & - \\ -\mathrm{X} \\ \mathrm{X} & -\mathrm{X} & \\ \mathrm{X} & - & \mathrm{X}\end{array}$

X

X

X

X

$\mathrm{X}$

$\mathrm{X} \quad \mathrm{X}$

$\mathrm{X}$

$X$

$\mathrm{X}$

X

$-$

X

$X$

?

X

$X$

$\mathrm{X}$

-

$\mathrm{X}$

$X$

X

$X$

X 


\section{Taxon}

C. viridis Philippine Coucal

Cuculus fugax Hodgson's Hawk-cuckoo

Surniculus lugubris Drongo Cuckoo

Family Tytonidae

Tyto capensis Grass Owl

Family Strigidae

Mimizuku gumeyi Giant Scops-owl

Ninox philippensis Philippine Hawk-owl

Otus megalotis Philippine Scops-owl

O. mirus Mindanao Scops-owl

Family Podargidae

Batrachostomus septimus Philippine Frogmouth

Family Eurostopidae

Eurostopodis macrotis Great Eared Nightjar

Family Caprimulgidae

Caprimulgus manillensis Philippine Nightjar

Family Apodidae

Collocalia amelis Island Swiftlet

C. esculenta Glossy Swiftlet

C. mearnsi Philippine Swiftlet

C. troglodytes Pygmy Swiftlet

Hirundapus celebensis Purple Needletail

$H$. giganteus Brown-backed Needletail

Mearnsia picina Philippine Needletail

Family Hemiprocnidae

Hemiprocne comata Lesser Treeswift

Family Trogonidae

Harpactes ardens Philippine Trogon

Family Coraciidae

Eurystomus orientalis Dollarbird

Family Alcedinae

Actenoides hombroni Blue-capped Woodkingfisher

Alcedo argentatus Silvery Kingfisher

A. atthis Common Kingfisher

Halcyon capensis Stork-billed Kingfisher

H. chloris White-collared Kingfisher

H. smymensis White-throated Kingfisher

Family Meropidae

Merops philippimus Blue-tailed Bee-eater

M. viridis Blue-throated Bee-eater

Family Bucerotidae

Aceros leucocephalus Writhed Hornbill

Buceros hydrocorax Rufous Hornbill

Penelopides affinis Mindanao Hornbillt

Family Capitonidae

Megalaima haemacephala Coppersmith Barbet

Family Picidae

Chrysocolaptes lucidus Greater Goldenback

Dendrocopos maculatus Philippine Pygmy

Woodpecker

Dryocopus javensis White-bellied Woodpecker

Family Eurylaimidae

Eurylaimus steerii Mindanao Broadbill

Family Pittidae

Pitta erythrogaster Red-bellied Pitta

Family Hirundinidae

Hirundo tahitica Pacific Swallow

Family Campephagidae

Coracina mcgregori McGregor's Cuckoo-shrike

C. morio Black-shouldered Cuckoo-shrike
Conserva- Restricted Recent Recent Historical Endemism tion status range records specimens specimens

$\begin{array}{llllll}\mathrm{P} & - & - & \mathrm{X} & - & \mathrm{X} \\ - & - & - & \mathrm{X} & \mathrm{X} & \mathrm{X} \\ - & - & - & \mathrm{X} & - & \mathrm{X}\end{array}$

$\begin{array}{cc}- & \\ \text { GM } & \text { VU } \\ \text { P } & - \\ \text { P } & \text { NT }\end{array}$

X

$\mathrm{P}$

$-$

$-$

$X$

$X$
$X$
$X$

$-$

$\mathrm{P}$

$\bar{P}$

$\mathrm{P}$

$-$

$\mathrm{P}$

NT

$\begin{array}{ccc}X & X & \\ - & - & X \\ X & X & X\end{array}$

$X$
$X$
$X$

X

X

X

X X X

$\mathrm{X}$

$\mathrm{X}$

X

X

X

p

$-$

M

VU

VU

$\mathrm{P}$

$-$

-

$-$

$-$

GM NT

$\mathrm{P}$

NT

$P$

$-$

X

X

X

$\mathrm{X}$

$\mathrm{X}$

$\mathrm{X}$

X

$\bar{X}$

X

X

X

X

$X$

X

$\mathrm{X}$

GM

VU

$\mathrm{X}$

$X$

X

$\mathrm{X}$

X

X 


\section{Taxon}

C. striata Bar-bellied Cuckoo-shrike

Lalage melanoleuca Black-and-white Triller

L. nigra Pied Triller

Pericrocotus flammeus Scarlet Minivet

Family Chloropseidae

Chloropsis flavipennis Philippine Leafbird

Family Pycnonotidae

Hypsipetes everetti Yellowish Bulbul

H. philippinus Philippine Bulbul

Pycnonotus goiavier Yellow-vented Bulbul

$P$. urostictus Yellow-wattled Bulbul

Family Dicuridae

Dicrurus hottentottus Spangled Drongo

Family Oriolidae

Irena cyanogaster Philippine Fairy-bluebird

Oriolus chinensis Black-naped Oriole

O. steerii Philippine Oriole

Family Corvidae

Corvus macrorhynchos Large-billed Crow

Family Paridae

Parus elegans Elegant Tit

Family Sittidae

Sitta oenochlamys Sulphur-billed Nuthatch $\dagger$

Family Rhabdornithidae

Rhabdornis inomatus Stripe-breasted Rhabdornis

R. mystacalis Stripe-headed Rhabdornis

Family Timaliidae

Leonardina woodi Bagobo Babbler

Macronous striaticeps Brown Tit-babbler

Micromacronous leytensis Miniature Tit-babbler

Ptilocichla mindanensis Streaked Groundbabbler

Stachyris capitalis Black-capped Babbler

S. plateni Pygmy Babbler

Family Turdidae

Brachypteryx montana White-browed Shortwing

Copsychus saularis Oriental Magpie robin

Saxicola caprata Pied Bushchat

Turdus poliocephalus Island Thrush

Zoothera andromedae Sunda Ground-thrush

Family Sylvidae

Bradypterus caudatus Long-tailed Ground-warbler Cisticola exilis Zitting Cisticola

C. juncidis Golden-capped Cisticola

Gerygone sulphurea Golden-bellied Flyeater

Megalurus palustris Striated Grassbird

M. timoriensis Tawny Grassbird

Orthotomus frontalis Rufous-fronted Tailorbird $\dagger$

$O$. heterolaemus Rufous-headed Tailorbird $\uparrow$

$O$. nigriceps Black-headed Tailorbird

Phylloscopus olivaceus Philippine Leaf-warbler

$P$. trivirgatus Mountain Leaf-warbler

Family Muscicapidae

Culicicapa heliantha Citrine Canary-flycatcher

Eumyias panayensis Mountain Verditer-flycatcher

Ficedula hyperythra Snowy-browed Flycatcher

F. westermanni Little Pied Flycatcher

Hypothymis azurea Black-naped Monarch

Rhinomyias goodfellowi Goodfellow's Jungleflycatcher
Conserva- Restricted Recent Recent Historical Endemism tion status range records specimens specimens

$P$

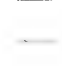

P

$\mathrm{P}$

$\mathrm{P}$

$\mathrm{P}$

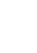

$\mathrm{P}$

$\bar{P}$

-

P

-

$\mathrm{P}$

$\mathrm{P}$

M

$\mathrm{P}$

GM

GM

$P$

GM

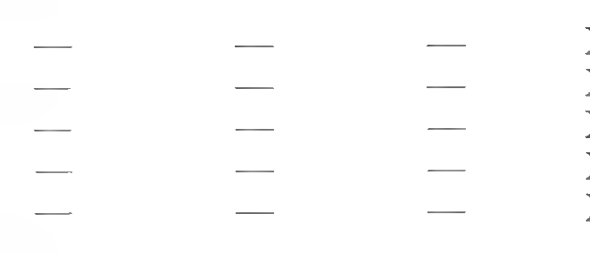

P

- $\quad-\quad-\quad-$

$-$

GM

M

GM

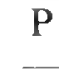

P

VU

$\mathrm{X}$

- $\quad \mathrm{X}$

$\mathrm{X} \quad \mathrm{X}$

$\mathrm{X}$
$\mathrm{X}$

$X$
$X$

$\mathrm{X}$

$\mathrm{X}$

$\mathrm{X}$

$\mathrm{X}$

$\mathrm{X}$

$\mathrm{X}-\mathrm{X}$

$\mathrm{X}$

- $\quad \mathrm{X}$

X

$\mathrm{X}$

$\mathrm{X}$

$\mathrm{X}$

$\mathrm{X}$

X $\quad \mathrm{X}$

$\mathrm{X}$

$\mathrm{X}$

$\mathrm{X}$

$\mathrm{X}$

$\mathrm{X}$

$\mathrm{X}$

$\mathrm{X}$

$\mathrm{X}$

$\begin{array}{lll}x & x\end{array}$

$\underset{X}{X}$

$\mathrm{X}$

DD

$\mathrm{X}$

-

X

X

X

$\mathrm{X}$

X X

$\mathrm{X}$

$\mathrm{X} \quad \mathrm{X}$

$-$

X

$\mathrm{X}$

$\begin{array}{lll}X & X & X \\ X & - & X\end{array}$

$\mathrm{X}$

$\bar{x}$

$\mathrm{X}$

$\mathrm{X}$

$\mathrm{X}$

$\mathrm{X}$

$\mathrm{X}$

X

$\begin{array}{ll}- & - \\ - & - \\ - & - \\ \bar{M} & \text { NT }\end{array}$

X

X

x

.

X

$\mathrm{X}$


TABLE 2. Continued.

\section{Taxon}

Conserva- Restricted Recent Recent Historical

Endemism tion status range records specimens specimens

Rhipidura nigrocinnamomea Black-and-cinnamon Fantail

$R$. superciliaris Blue Fantail

Family Pachycephalidae

Pachycephala philippensis Yellow-bellied Whistler

Family Motacillidae

Anthus rufulus Paddyfield Pipit

Family Artamidae

Artamus leucorhynchus White-breasted WoodSwallow

Family Laniidae

Lanius schach Mountain Shrike

Lanius validirostris Long-tailed Shrike

Family Sturnidae

Aplonis minor Short-tailed Glossy Starling

A. panayensis Asian Glossy Starling

Basilornis miranda Apo Myna

Sarcops calvus Coleto

Family Nectarinidae

Aethopyga boltoni Apo Sunbird

A. primigenius Grey-hooded Sunbird

$A$. pulcherrima Metallic-winged Sunbird

A. shelleyi Lovely Sunbird

Anthreptes malacensis Plain-throated Sunbird

Arachnothera clarae Naked-faced Spiderhunter

A. longirostra Little Spiderhunter

Nectarinia jugularis Olive-backed Sunbird

$N$. sperata Purple-throated Sunbird

Family Dicaedae

Dicaeum aeruginosum Striped Flowerpecker

D. anthonyi Flame-crowned Flowerpecker

D. australe Red-keeled Flowerpecker

D. bicolor Bicolored Flowerpecker

D. hypoleucum Buzzing Flowerpecker

D. ignipectus Fire-breasted Flowerpecker

D. nigrilore Olive-capped Flowerpecker

D. proprium Whiskered Flowerpecker

D. pygmaeum Pygmy Flowerpecker

M

GM

$\mathrm{P}$

$\mathrm{P}$

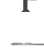

D. trigonostigma Orange-bellied Flowerpecker Prionichilus olivaceus Olive-bellied Flowerpecker

Family Zosteropidae

Hypocryptadius cimnamomeus Cinnamon Ibon

Lophozosterops goodfellowi Black-masked Whiteeye

Zosterops everetti Everett's White-eye

$Z$. montanus Mountain White-eye

Family Ploceidae

Passer montanus Eurasian Tree Sparrow

Family Estrildidae

Erythrura coloria Red-eared Parrotfinch

Lonchura leucogastra White-bellied Munia

L. malacca Chestnut Munia

L. punctulata Scaly-breasted Munia

Family Fringillidae

Pyrrhula leucogenys White-cheeked Bullfinch

Serimus estherae Mountain Serin

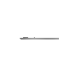

$-$

$$
\overline{-}
$$

X

X

X

X

X X

X

X

X

\section{NT}

X

X

X

X

X$$
\text { - }
$$$$
-
$$

$\mathrm{X}$
$\mathrm{X}$

X

X

$\begin{array}{llll}- & X & - & X \\ - & X & - & X \\ X & X & X & X \\ - & X & - & X\end{array}$

X 
TABLE 3. Summary of information on migratory bird species known from Mt. Kitanglad. Shown are early and late dates for each species, plus A: recent specimens (1992-1993): B: historical specimens (Rabor and colleagues).

\begin{tabular}{|c|c|c|c|c|}
\hline Taxon & Earliest date & Latest date & $\mathbf{A}$ & B \\
\hline \multicolumn{5}{|l|}{ Family Accipitridae } \\
\hline Accipiter gularis Japanese Sparrowhawk & late Sep. (Table In) & 15 Mar. (Table 1d) & - & - \\
\hline A. soloensis Chinese Goshawk & late Feb. (Table $1 \mathrm{~m}$ ) & 3 Apr. $[\mathrm{AG}]$ & - & - \\
\hline Butastur indicus Grey-faced Buzzard & 7 Dec. (Table $1 \mathrm{~g}$ ) & 15 Mar. (Table Id) & - & $\mathrm{X}$ \\
\hline Circus melanoleucos Pied Harrier & 3 Oct. [TB, GD] & early Apr. (Table la) & - & - \\
\hline C. spilonotus Eastern Marsh-harrier & 26 Feb. (Table $1 \mathrm{~m}$ ) & 13 Mar. $[\mathrm{AG}]$ & - & - \\
\hline \multicolumn{5}{|l|}{ Family Falconidae } \\
\hline Falco timmunculus Eurasian Kestrel & & $19 \operatorname{Mar} .[\mathrm{AG}]$ & - & - \\
\hline F. peregrinus Peregrine Falcon & 3 Dec. (Table 1g) & $23 \mathrm{Apr} .[\mathrm{AG}]$ & - & - \\
\hline \multicolumn{5}{|l|}{ Family Scolopacidae } \\
\hline Gallinago megala Swinhoe's Snipe & 6 Oct. $[\mathrm{TB}, \mathrm{GD}]$ & 8 Apr. [AG] & - & - \\
\hline \multicolumn{5}{|l|}{ Family Cuculidae } \\
\hline Cuculus saturatus Oriental Cuckoo & $19 \operatorname{Mar} .[\mathrm{AG}]$ & May $[\mathrm{ATP}, \mathrm{RF}]$ & $\mathrm{X}$ & $\mathrm{X}$ \\
\hline \multicolumn{5}{|l|}{ Family Hirundinidae } \\
\hline Hirundo rustica Barn Swallow & 4 Oct. [TB, GD] & 8 Apr. $[\mathrm{AG}]$ & - & $\mathrm{X}$ \\
\hline H. striolata Striated Swallow & & early Apr. (Table 1a) & - & $\mathrm{X}$ \\
\hline \multicolumn{5}{|l|}{ Family Turdidae } \\
\hline Luscinia calliope Siberian Rubythroat & late Sep. (Table $1 \mathrm{n}$ ) & - & - & - \\
\hline Monticola solitarius Blue Rock-Thrush & 10 Oct. (zMuc 176) & - & - & $\mathrm{X}$ \\
\hline Turdus chrysolaus Brown-headed Thrush & & 13 Mar. (Table li) & - & - \\
\hline T. obscurus Eye-browed Thrush & 2 Dec. (Table $1 \mathrm{~g}$ ) & $21 \mathrm{Apr} .[\mathrm{AG}]$ & $\mathrm{X}$ & - \\
\hline \multicolumn{5}{|l|}{ Family Sylvidae } \\
\hline $\begin{array}{l}\text { Acrocephalus arundinaceus Great Reed- } \\
\text { warbler }\end{array}$ & Dec. (SMF 47194) & - & - & $\mathrm{X}$ \\
\hline $\begin{array}{l}\text { Locustella fasciolata Gray's Grasshopper- } \\
\text { warbler }\end{array}$ & - & 2 Jan. $[A G]$ & - & - \\
\hline Phylloscopus borealis Arctic Warbler & late Sep. (Table $1 \mathrm{n}$ ) & $\begin{array}{l}24 \text { May (Ripley \& } \\
\text { Rabor, 1961) }\end{array}$ & & \\
\hline \multicolumn{5}{|l|}{ Family Muscicapidae } \\
\hline Ficedula mugimaki Mugimaki Flycatcher & late Sep. (Table 1n) & 15 Mar. (Table 1j) & $\mathrm{X}$ & - \\
\hline $\begin{array}{l}\text { Muscicapa griseisticta } \text { Grey-streaked } \\
\text { Flycatcher }\end{array}$ & late Sep. (Table ln) & $\begin{array}{l}\text { I May (Ripley \& } \\
\text { Rabor, 1961) }\end{array}$ & $\mathrm{X}$ & - \\
\hline \multicolumn{5}{|l|}{ Family Motacillidae } \\
\hline Anthus gustavi Pechora Pipit & 29 Feb. (Table $1 \mathrm{k}$ ) & 19 Apr. [ATP, RF] & $\mathrm{X}$ & $\mathrm{X}$ \\
\hline A. hodgsoni Olive Tree-pipit & 15 Dec. (Table 1o) & 6 Apr. (YPM) & $\mathrm{X}$ & - \\
\hline Motacilla alba White Wagtail & 20 Apr. $[A G]$ & 25 Apr. [AG] & - & - \\
\hline M. cinerea Grey Wagtail & late Sep. (Table In) & 1 May (Table 11) & $\mathrm{X}$ & - \\
\hline M. flava Yellow Wagtail & 4 Dec. (Table 1g) & Apr. [ATP, RF] & - & - \\
\hline \multicolumn{5}{|l|}{ Family Laniidae } \\
\hline Lanius cristatus Brown Shrike & late Sep. (Table 1n) & 1 May (Table 11) & $\mathrm{X}$ & - \\
\hline
\end{tabular}

species from Kitanglad. Birds probably of this species were reported on 6 Oct. 1991 [TB, GD], 14 Mar. 1993, 15 Jan. 1994, and 8 Apr. 1994 (2 birds) at $860-1020 \mathrm{~m}$ in grasslands on the northeast slope $[\mathrm{AG}]$ and on the lower slopes in Feb. 1994 by M. Archer (Table 1f).

\section{Scolopax bukidnonensis Bukidnon Woodcock $\dagger$}

Woodcocks have recently been discovered on Kitanglad. Several individuals have been collected from the mountain as well as from elsewhere in the Philippines, and the form has recently been described as a species new to science (Kennedy et al., 2001). In addition to the small series of specimens on which the species description was based, another (SMF uncataloged) is from Bo Kaatuan, at about $1500 \mathrm{~m}$.

Birds were first seen in Jan. 1992 (R. Timmins, pers. comm., 1992), seen well on 18-20 Feb. 1993 (Robson, 1993; Harrap \& Fisher, 1994), and tape-recorded in late Feb. 1993 (Harrap \& Fisher, 1994). Subsequent sightings include birds seen in Mar. 1993 and Jan. 1994 [AG, TB], Jan.Feb. 1994 (Harrap \& Fisher, 1994; Table 1f), Apr. 1994 [AG, TB], 15-20 Dec. 1994 and 11-12 Jan. 1995 (Table 1o), Dec. 1996 (Table 1b), and 2-12 Dec. 1998 (Table 1g). The lack of sightings from other months suggests that peak display 
period may be Dec.-Feb. (T. Fisher sought it specifically but did not see or hear birds, on 25 27 Mar. 1994; Harrap \& Fisher, 1994). At higher elevations on the northeast slope, AG saw a bird in a burned area at $1800 \mathrm{~m}$ on the east slope of Mt. Tuminungan in Apr. 1994 and noted an individual (tentatively identified as S. rusticola but probably also referable to this taxon) at $1450 \mathrm{~m}$ on a grassy shoulder on 7 Jan. 1994. SEB and DHC collected 2 at 1455-1465 $\mathrm{m}$ in Apr. 1999,1 in the evening and 1 at dawn, both in canopy nets.

Descriptions indicate that the birds' call is a "distinctive 'rattle' repeated continuously in flight as it displayed over the same route every day ... regularly heard giving rapid bursts of high pitched 'machine-gun fire' of ca. 1.5 second duration, separated by gaps of 3 seconds" (Harrap \& Fisher, 1994; Table 1o). It usually flew too low against dark vegetation and in too low light levels to be seen. Display flights were between 05:15 and 05:20 h most mornings and $17: 50$ and $18: 05 \mathrm{~h}$ on only 2 evenings in late December (Table 1b).

\section{Phapitreron leucotis White-eared Brown-dove}

The genus Phapitreron presents a complex situation on Kitanglad. Phapitreron leucotis is well represented in collections (DMNH, FMNH, PNM, YPM, SMF) (Ripley \& Rabor, 1961) from the mountain. In Apr.-May 1992, ATP, JCTG, and NADM found it at low elevations around San Vicente and at 1100-1300 m (FMNH 357397-357400); in 1993 and 1994, it was found frequently at $590-900 \mathrm{~m}$ on the northwest slope and regularly between 1100 and $1200 \mathrm{~m}$ and occasionally up to $1450 \mathrm{~m}$ on the northeast slope [AG]. SEB and DHC collected 3 at $1555-1570 \mathrm{~m}$ in Apr.-May 1999. Other sight records of this species, which is common elsewhere in the Philippines (Dickinson et al., 1991), are few: individuals on 29 Apr.-1 May 1983 (Table 11) and several in Dec. 1996 (Table 1b). Possibly these birds are hunted in the accessible forest above Dalwangan.

\section{Phapitreron amethystina Amethyst Brown-dove}

Like the preceding species, this species is well represented in collections from Kitanglad (DMNH, FMNH, PNM, YPM, SMF) (Ripley \& Rabor, 1961) but less well by sight records. We found it at 1100 $1800 \mathrm{~m}$ (FMNH 357401-9) in 1992 [ATP, RF], mistnetted 2 at $1900 \mathrm{~m}$ and sighted others up to $2250 \mathrm{~m}$ on Mt. Nangkabulos in Mar. 1993 [JCTG, NADM], and found birds regularly in secondary montane and mossy forests between 1300 and $1780 \mathrm{~m}$ on the northeast slope and at $900-1100 \mathrm{~m}$ on the northwest slope in 1993-1994 [AG]. SEB and DHC collected 5 at $1465-1570 \mathrm{~m}$ in Apr.May 1999. The only other sight records are from 29 Apr.-1 May 1983 (Table 11) and Dec. 1996 (Table 1b), so possibly this species also suffers from heavy hunting pressure above Dalwangan.

\section{Phapitreron brumneiceps Mindanao Brown-dove $\dagger$ VULNERABLE}

The Mindanao populations of this rare species, which are restricted to montane areas (Dickinson et al., 1991), have recently been split from the lowland populations of the Sulu Islands (Collar et al., 1999). The species was reported from Kitanglad (duPont, 1971), but the specimen available from the mountain (DMNH 68709) in the Delaware Natural History Museum was rejected as a viable voucher by Dickinson et al. (1991). Additional individuals were collected (FMNH 357410-2) at 1100-1500 $\mathrm{m}$ in Apr. 1992 [ATP, JCTG, NADM], and it was also sighted in 1995 (A. Long and M. Heath, in litt., 1995). Hence, recent work confirms the presence of this species on the Kitanglad massif.

\section{Ducula poliocephala Pink-bellied Imperial-pigeon \\ NEAR THREATENED}

This species is known from Kitanglad from our sightings only: at 1100-1800 $\mathrm{m}$ in Apr.-May 1992 [ATP, RF] and at $1260-1540 \mathrm{~m}$ on the northeast slope between Jan. and Apr. 1994 and on Mt. Tuminungan at $1780 \mathrm{~m}$ on $10 \mathrm{Apr} .1994$ [AG]. Local people reported that the species is hunted heavily, and our observations of its extreme flightiness suggest that such is indeed the case. This may explain the paucity of records and also supports the inclusion of this previously unlisted species as near threatened (Collar et al., 1999).

\section{Ducula carola Spotted Imperial-pigeon}

VULNERABLE

Several historical specimens place this species on the mountain in 1960 (DMNH 13621-13622, YPM 61608-61612) (Ripley \& Rabor, 1961) and 1965 (SMF 47095), but only 1 recent report exists, from Feb. 1991 (Table 1m). The species may be partially nomadic (Collar et al., 1999), which might explain its presence in reasonable numbers in March-April (Ripley \& Rabor, 1961). However, given that recent visits to Kitanglad were concentrated in these same months, this species may have declined on the mountain. 


\section{Columba vitiensis Metallic Pigeon}

This species was collected on Kitanglad in 16 Apr.-9 May 1960 (FMNH 422617, YPM 6161361619) (Ripley \& Rabor, 1961), but the only recent records are sightings of 1 on 13 Mar. 1990 (Table 1d) and 2 on 10 Feb. 1994 (Table 1f). The paucity of recent records suggests that this species, along with many of the Philippines' large forest pigeons, is in rapid decline.

\section{Streptopelia bitorquata Island Turtle-dove}

The only Kitanglad record of this species is a specimen from 20 Dec. 1960 (YPM 62294), with no recent records. This species appears to be in serious decline, perhaps because of competition from Spotted Doves ( $S$. chinensis), which are spreading explosively in the Philippines (Waldbauer \& Waldbauer, 1982).

\section{Trichoglossus johnstoniae Mindanao Lorikeet NEAR THREATENED}

Numerous specimens place this species on Kitanglad (ANSP, FMNH, PNM, YPM) (Ripley \& Rabor, 1961); sight records are as follows: 12-15 birds on 13 Mar. 1987 (Table 1j), 2 on 15 Mar. 1990 (Table 1d), and sightings [GD] on 4 Oct. 1991 (Evans et al., 1993), in Feb. 1993 (I. Gardner in litt., 1993), at $1900 \mathrm{~m}$ on Mt. Nangkabulos in Mar. 1993 [JCTG, NADM], single individuals or pairs at $1160-1300 \mathrm{~m}$ on the Dalwangan site and at $590-720 \mathrm{~m}$ in the Kalawaig valley in both 1993 and 1994 [AG], and several at $1250 \mathrm{~m}$ on 8 Dec. 1998 (Table $1 \mathrm{~g}$ ). Hence, the species may have declined on Kitanglad, given that lorikeets should be fairly easily to observe (Juniper \& Parr, 1998). Its status elsewhere on Mindanao, though, appears more secure than previously thought, and Collar et al. (1999) down-listed the taxon to near threatened from vulnerable.

\section{Bolbopsittacus lunulatus Guiabero}

This Philippine lowland endemic species is generally common (Dickinson et al., 1991) but is known from Kitanglad only from a handful of sight records: on fruit-bearing trees in the Lalawan valley at $900 \mathrm{~m}$ (16 Mar. 1993) and $1290 \mathrm{~m}$ (4 Jan. 1994) on the northeast slope and at $650-840 \mathrm{~m}$ on the northwest slope in Apr. 1994 [AG] and in Mar. 1990 (Table 1d).

\section{Prioniturus discurus Blue-crowned Racquet-tail}

This species is scarce on Kitanglad, where forests are principally at or above the upper limit of its altitudinal range (Dickinson et al., 1991).
Elsewhere in the Philippines, it appears more common than previously thought and so is no longer considered near threatened (Collar et al., 1999). It has been found mostly at middle elevations: at $1200-1460 \mathrm{~m}$ on the northeast slope in 1993-1994 [AG], a group of 4 individuals in lowland forest at $650 \mathrm{~m}$ on the northwest slope on 19 Apr. 1994 [AG], and "17+ in flocks 15 Mar" in 1987 (Table 1j). Several old specimens also exist from the mountain (PNM 10126 10129, Y РМ 62307-62309), including several from a high 1400-1500 m (e.g., FMNH 258646).

\section{Prioniturus waterstradti Mindanao Racquet-tail $\dagger$ NEAR THREATENED}

Mindanao populations of this taxon are considered a species separate from $P$. montanus of Luzon by some workers (Sibley \& Monroe, 1990; Juniper \& Parr, 1998). At least 11 specimens exist from Kitanglad (YPM 61660-61665; SMF 47008-47009, 47011-47012, 48161; FMNH 262472, $357417-$ 357419, 392241) (Ripley \& Rabor, 1961), and we noted 2-5 individuals daily on $4-6$ Oct. 1991 [TB, GD], flocks daily at $1100-1800 \mathrm{~m}$ in Apr.-May 1992 [ATP, RF], at $2250 \mathrm{~m}$ on Mt. Nangkabulos in Mar.-Apr. 1993 [JCTG, NADM], and 2-6 at $1420-1800 \mathrm{~m}$ on the northeast slope in Apr. 1993 and 1994 [AG]. SEB and DHC collected 1 at $1465 \mathrm{~m}$ in Apr. 1999. Other sightings include small groups on 29 Apr.-1 May 1983 (Table 11), 13-14 Mar. 1987 (Table 1j), 11-14 Mar. 1990 (Table 1d), late Sep. 1994 (Table 1n), 17 Dec. 1994 and 11 Jan. 1995 (Table 1o), 4 Apr. 1996 (Table 1a), Dec. 1996 (Table 1b), 6-7 Apr. 1998 (Table 1h), and 2-10 Dec. 1998 (Table 1g). This frequency of records suggests that Collar et al. (1999) were right to down-list this taxon to near threatened from vulnerable (Collar et al., 1994).

\section{Cuculus saturatus Oriental Cuckoo}

The species appears to be a shy and silent winter resident on Kitanglad, as most records have involved birds caught in mist-nets. A female was collected on 7 Apr. 1960 (YPM 61673) (Ripley \& Rabor, 1961) at $<1670 \mathrm{~m}$, and birds were mist-netted (FMNH 357422-357423) in Apr. 1992 at $1100 \mathrm{~m}$ and in May 1992 at $1500 \mathrm{~m}$ [ATP, $\mathrm{RF}]$. The only other records are from 19 Mar. 1993 at $1460 \mathrm{~m}$ on the northeast slope and from 25 to 26 Mar. 1993 and 25 Apr. 1994 at 590$610 \mathrm{~m}$ on the northwest slope [AG].

\section{Surniculus lugubris Drongo Cuckoo}

Perhaps because of its elusiveness, this species is known on Kitanglad from only 1 specimen 
from Cabanglasan at the mountain's foot from 19 Oct. 1951 (ZMUC 1672), 1 specimen from 9 May 1960 (YPM 61680) (Ripley \& Rabor, 1961), and 1 probable sighting in primary forest at $1580 \mathrm{~m}$ on the northeast slope on 4 Apr. 1994 [AG]. Philippine populations of this species have most recently been treated as a separate species, S. velutinus (Payne, 1997).

\section{Centropus melanops Black-faced Coucal}

This Greater Mindanao endemic is generally considered a lowland species-Dickinson et al. (1991) gave its maximum elevation as $1200 \mathrm{~m}$. However, we never saw it on the 1300 -m ridge or below, but it was heard daily at $1500-1800 \mathrm{~m}$ in Apr.-May 1992 [ATP, RF] and sighted briefly and at long distances on several occasions. It was also sighted regularly at $1420-1560 \mathrm{~m}$ on the northeast slope in 1993 and 1994 [AG]; Jensen and Hornskov (Table 1j) heard 1 on 15 Mar. 1987. No specimens are-to our knowledgeavailable of this taxon from the Kitanglad region. The contrast in apparent altitudinal ranges begs further investigation into the ecological characteristics of the Kitanglad population, which could conceivably represent a population distinct from the lowland forms.

\section{Otus mirus Mindanao Scops-owl}

\section{NEAR THREATENED}

This Mindanao endemic was previously known only from Mt. Hilong-Hilong and Mt. Apo (Dickinson et al., 1991) but in May 1992 was found to be common and vocal from $1500 \mathrm{~m}$ upward on Kitanglad, with numerous individuals (FMNH 357428-357430) mist-netted [ATP, RF]. It was subsequently found at $1900-2250 \mathrm{~m}$ on Mt. Nangkabulos in Mar.-Apr. 1993 [JCTG, NADM], calling at $1200 \mathrm{~m}$ at a single site in the Lalawan valley on the northeast slope in 1993 and 1994 [AG], and at $1455 \mathrm{~m}$ in Apr. 1999 [SEB, DHC]. It was also reported heard in Feb. 1991 (Table lm), in Feb. 1992 (Table 1k), and probably in late Sep. 1994 (Table 1n). The odd "dove-like couplet" call of the species, described by J. T. Marshall (Dickinson et al., 1991), is occasionally replaced by a more typical Otus tremulo in the hand [ATP]. Collar et al. (1999) down-listed the species to near threatened from vulnerable, and the discovery of this Kitanglad population adds strength to this decision.

\section{Otus megalotis Philippine Scops-owl}

Otus megalotis appears uncommon and retiring on Kitanglad, as it was detected on only on
5 days in Apr.-May 1992 at 1100-1500 m [ATP, $\mathrm{RF}$ ], including adults mist-netted at $1100 \mathrm{~m}$ and a fledgling collected at $1400 \mathrm{~m}$ (FMNH 357431357433). In Mar. 1993 and Apr. 1994, it was heard calling at $1150-1270 \mathrm{~m}$ at the Dalwangan site, where it was also seen on 5 Jan. 1994 [AG]. Another sight record comes from 22 Apr. 1994 near Landut, at $980 \mathrm{~m}$ on the northwest flank [AG]. The only other records are sightings on 26-28 Feb. 1992 (Table 1k) and specimens collected in 1960 at $1400 \mathrm{~m}$ (YPM 61681-2) (Ripley \& Rabor, 1961) and in 1964-1965 at $1500 \mathrm{~m}$ (SMF 47037-42).

\section{Mimizuku gurneyi Giant Scops-owl}

VULNERABLE

This rare species is known on Kitanglad from a few specimens collected at $1300 \mathrm{~m}$ on 6 May 1960 (YPM 61683) (Ripley \& Rabor, 1961) and at $1500 \mathrm{~m}$ in 1964-1965 (sMF 47027-47028, 81991). Many recent sightings exist, however, including birds calling at $600-630 \mathrm{~m}$ on the northwest slope on 23-25 Apr. 1994 [AG]. On the northeast flank of the mountain, records include birds seen and heard on 13-16 Mar. 1990 (Table 1d), 28 Feb. 1991 (Table 1m), 27-28 Feb. 1992 (Table 1k), in Feb. 1993 (I. Gardner, in litt., 1993), calling at 20:00-21:00 $\mathrm{h}$ and 01:00 $\mathrm{h}$ and seen once at $1200-1250 \mathrm{~m}$ in both 1993 and 1994 [AG], Jan. 1994 and Feb. 1997 by P. Morris and Apr. 1994 by P. Davidson (Collar et al., 1999), 18 Dec. 1994 (Table 1o), Dec. 1996 at $1250 \mathrm{~m}$ (Table 1b), 3 Dec. 1998 at $1250 \mathrm{~m}$ (Table 1g), and aural records from TB and GD on 4 Oct. 1991 (Evans et al., 1993), 7 Feb. 1994 (Table 1f), and in Apr. 1996 (Table 1a). Although presumably at the upper limits of its "low and middle elevation" altitudinal range on Kitanglad (Miranda et al., 1997), the frequency of records from the site support the judgment of Collar et al. (1999) to list the species as vulnerable rather than endangered.

\section{Ninox philippensis Philippine Hawk-owl}

A single specimen from the 1960s (smF 47029; Bo Kaatuan) and an aural record of this species from late Sep. 1994 (Table ln) are the only documentation of its presence on Kitanglad. The paucity of records from the mountain is quite surprising.

\section{Collocalia mearnsilamelis/vanikorensis† Philippine/ Island Swiftlet}

The taxonomy (Dickinson, 1989) and identification (Chantler \& Driessens, 1994) of large, 
dark swiftlets in the Philippines is extremely problematic, as indeed is the name to which this form should be assigned (referred to as $C$. amelis in the text and tables that follow but may be better considered within $C$. vanikorensis). Such swiftlets are common on Kitanglad and are likely to be predominantly $C$. mearnsi, an uncommon Philippine montane endemic (Dickinson et al., 1991). The only conclusive documentation of this, however, are specimens collected at Kibangay at $1260 \mathrm{~m}$ on $21 \mathrm{Dec} 1951$ by Salamonsen (zMUC 1684), 1 collected at Malaybalay on 11 Oct. 1951 (ZMUC 1686), and 1 collected from a cave at $1620 \mathrm{~m}$ in May 1999 by SEB and DHC. Numerous reports of Grey Swiftlet C. amelis, a taxon given full specific status by Sibley and Monroe (1990), could certainly represent birds wandering up onto Kitanglad from the adjacent lowlands-a specimen exists from Malaybalay from 12 Oct, 1951 (ZMUC 225) - but no clear criteria are known for separating the 2 in the field (Chantler \& Driessens, 1994). AG and colleagues saw swiftlets identified as $C$. amelis at $900-1500 \mathrm{~m}$ on the northeast slope, with excellent details and comparisons; members of this species were often in mixed flocks with $C$. esculenta. Sight records of large, dark swiftlets on Kitanglad are numerous (Table $1 \mathrm{~d}, \mathrm{f}-\mathrm{h}, \mathrm{j}-\mathrm{l}, \mathrm{o}$ ) but lack sufficient detail to permit definitive determination. Lack of broader specimen documentation makes conclusive statements regarding the occurrence of these 2 taxa on Kitanglad difficult.

\section{Collocalia esculenta Glossy Swiftlet}

DHC visited a small nesting colony in a cave along a stream about $15 \mathrm{~min}$ from Lupiagan (1300-1400 m). The cave was apparently manmade and apparently once held a larger colony that is now reduced in numbers. On 3 May 1999, DHC found 6 nests, of which 2 were empty, 2 held 2 eggs each, and 2 held nestlings. Swiftlets of this species were observed [DHC, ATP] foraging by picking insects off of lichens on canopy tree branches. Both DHC and ATP observed swiftlets of this species participating in multispecific foraging flocks.

\section{Mearnsia picina Philippine Needletail}

\section{NEAR THREATENED}

The status of this southern Philippine endemic (Dickinson et al., 1991) was recently up-listed to near threatened (Collar et al., 1999). It appears to be rather scarce on Kitanglad, with the only sightings being from below $800 \mathrm{~m}$ in Apr. 1992 [ATP], 4 in Mar. 1993 and 9 in Apr. 1994 between 660 and
$700 \mathrm{~m}$ on the northwest flank and 6 in Jan. 1994 at $1080-1100 \mathrm{~m}$ on the northeast flank [AG], from Sep. 1994 (Table 1n), 2 in Dec. 1996 (Table 1b), 2 at $1520 \mathrm{~m}$ in Apr. 1998 (Table $1 \mathrm{~h}$ ), and 1 at $1250 \mathrm{~m}$ on 2 Dec. 1998 (Table 1g).

\section{Harpactes ardens Philippine Trogon}

This species is represented from Kitanglad by numerous specimens (FSM, PNM, YPM, ZMUC) (Ripley \& Rabor, 1961), and we found it at 700$1800 \mathrm{~m}$ in Apr.-May 1993 [ATP, RF], at $1900 \mathrm{~m}$ on Mt. Nangkabulos in Mar. 1993 [JCTG, NADM], and at $600-840 \mathrm{~m}$ on the northwest slope in well-structured secondary forest with canopy cover $>50 \%$ [AG]. The fact that no records exist from the northeast slope above Dalwangan suggest that the species may have declined dramatically in more accessible areas.

\section{Eurystomus orientalis Dollarbird}

No recent reports place this species on Kitanglad, the only records being specimens from the 1960s (YPM 61699-701, 62336-7, SMF 46924-5, 46927) (Ripley \& Rabor, 1961). A 1951 specimen is from Cabanglasan, at the mountain's foot (ZMUC 657).

\section{Alcedo argentata Silvery Kingfisher}

VULNERABLE

Although no records of this lowland Greater Mindanao endemic exist from the mountain itself (Dickinson et al., 1991), several 1951 specimens from Cabanglasan and the River Bubunaon place this species at its foot (ZMUC 741-747). Recent evidence for the survival of this population was provided by $\mathrm{P}$. Thompson (in litt., 1999), who found the species at Impalatao in 1999. Collar et al. (1999) considered a report of this lowland species from Dalwangan as hypothetical.

\section{Actenoides hombroni Blue-capped Wood-kingfisher VULNERABLE}

This species has only recently been sighted on Kitanglad, with 2 sightings by J. de Roever in Feb. 1991 (Table 1m), sightings by P. Davidson in Apr. 1994 and P. Morris in Feb. 1996 and Feb. 1997 (Collar et al., 1999), and birds seen daily on 3-5 Apr. 1996 (Table 1a). In addition, Mark (Table 1n) reported 1 in Sep. 1994, and Gee (Table $1 b$ ) reported an aural record: “... calls pre-dawn and usually only once. Song is a series of quite loud plew ... plew ... plew etc." An interesting low-elevation historical specimen exists from $\sim 400 \mathrm{~m}$ at Cabanglasan, Bukidnon, from 26 Oct. 1951 (ZMUC 1285). 


\section{Penelopides affinis Mindanao Hornbill $\dagger$}

Species limits of Penelopides in the Philippines are under debate, with some (Dickinson et al., 1991) treating all forms as panini, others (Kemp, 1988) treating the complex as 4 species (including affinis of Mindanao and the Eastern Visayas), and still others (Sibley \& Monroe, 1990; Collar et al., 1994) further separating samarensis of the Eastern Visayas from affinis. Collar et al. (1999) reverted to the treatment proposed by Kemp (1988) and hence dropped the taxon from consideration as near threatened. This decision is supported by the frequency of sight records from Kitanglad, including birds seen in late Apr. 1983 (Table 11), daily on 14-16 Mar. 1987 (Table 1i), in Oct. 1991 [TB, GD], on 26 Feb. 1991 (Table $1 \mathrm{~m}$ ), heard on 28 Feb. 1992 (Table 1k), at $1100 \mathrm{~m}$ in Apr. 1992 [ATP, RF], at $1900 \mathrm{~m}$ on Mt. Nangkabulos in Mar. 1993 [JCTG, NADM], regularly in 1993 and 1994 at $1180-1500 \mathrm{~m}$ on the northeast slope, and at $640-780 \mathrm{~m}$ on the northwest slope [AG], in late Sep. 1994 (Table 1n), on 16 Dec. 1994 (Table 1o), on 3 Apr. 1996 (Table 1a), in Dec. 1996 at $1250 \mathrm{~m}$ (Table 1b), on 6-7 Apr. 1998 at $1250-1520 \mathrm{~m}$ (Table $1 \mathrm{~h}$ ), and 2 on 2 Dec. and 3 on 8 Dec. 1998 at $1250 \mathrm{~m}$ (Table $1 \mathrm{~g}$ ). The taxon is also known from numerous Kitanglad specimens (DMNH, FMNH, PNM, YPM) (Ripley \& Rabor, 1961).

\section{Aceros leucocephalus Writhed Hornbill $\dagger$ \\ NEAR THREATENED}

This lowland Greater Mindanao endemic (Dickinson et al., 1991) is now considered specifically distinct from the Visayan Hornbill $A$. waldeni (Kemp 1988; Sibley \& Monroe, 1990; Kennedy et al., 2000). Many specimens were collected on Kitanglad in the 1960s (FMNH, PNM, YPM, SMF) (Ripley \& Rabor, 1961), and we sighted it at 1100-1200 m in Apr. 1992 [ATP, RF] and at $600-1100 \mathrm{~m}$ in the Kalawaig area in 1993-1994 [AG]. Like Buceros hydrocorax, no recent records exist from Kitanglad's accessible northeastern flank, raising serious concerns regarding the species' conservation status here. This point suggests that the decision by Collar et al. (1999) to down-list the taxon to near threatened from vulnerable may have been premature.

\section{Buceros hydrocorax Rufous Hornbill}

NEAR THREATENED

Although B. hydrocorax is not documented from Kitanglad by any specimens, we found it at
1100-1800 $\mathrm{m}$ in Apr.-May 1992 [ATP, RF] and at $1900-2250 \mathrm{~m}$ on Mt. Nangkabulos in Mar.Apr. 1993 [JCTG, NADM]. AG sighted this species only rarely, at lower elevations: once at $1460 \mathrm{~m}$ on the northeast slope on 6 Jan. 1994 and at $720-780 \mathrm{~m}$ on the northwest slope on 29 Mar. 1993 and 25 Apr. 1994 [AG]. The paucity of records from the accessible flanks of Kitanglad may indicate that the species has been extirpated from more accessible areas.

\section{Dryocopus javensis White-bellied Woodpecker}

An active nest with large young was found on 27 Apr. 1992 at $1100 \mathrm{~m}$; details are provided elsewhere (Peterson et al., 1995).

\section{Eurylaimus steerii Mindanao Broadbill}

VULNERABLE

The only Kitanglad record is of I collected at Cabanglasan at the mountain's base on 31 Oct. 1951 by Salomonsen (ZMUC 157) (Collar et al., 1999). Lambert (1996) recently separated the East Visayas form samarensis from this taxon.

\section{Pitta erythrogaster Red-bellied Pitta}

Although this species is present across the lowlands and foothills of Mindanao (Dickinson et al., 1991), the only sightings from Kitanglad are from the northwest slope: 1 on 26 Mar. 1993 in primary forest at $700 \mathrm{~m}$ and 2 in second growth with sparse ground cover at $650-750 \mathrm{~m}$ on 24 Apr. 1994 [AG].

\section{Coracina striata Bar-bellied Cuckoo-shrike}

This species is known from Kitanglad from only a few records: 2 specimens from the mountain's base from 1951 (ZMUC 481 482), 5 specimens from the 1960s (FMNH 262485-262486, YPM 62361-62362, SMF 46468) (Ripley \& Rabor, 1961), a single sighting at $700 \mathrm{~m}$ in Apr. 1992 [ATP, RF], and observations in the Lalawan valley at $950-1350 \mathrm{~m}$ and at $870 \mathrm{~m}$ between Kalawaig and Tulohan River [AG].

\section{Coracina mindanensis Black-bibbed Cuckoo-shrike} VULNERABLE

The only Kitanglad record of this lowland species is of 1 collected at the mountain's base at Cabanglasan on 27 Oct. 1951 by Salomonsen (zMUC 407).

\section{Coracina mcgregori McGregor's Cuckoo-shrike NEAR THREATENED}

Although Kitanglad is 1 of only 2 mountain ranges from which this species is known, it is common, and Collar et al. (1999) down-listed it 
from vulnerable to near threatened. Numerous specimens document its presence on the mountain (DMNH, FMNH, PNM, YPM, ZMUC, SMF) (Salomonsen, 1953; Ripley \& Rabor, 1961). We sighted 1-4 birds daily on 4-6 Oct. 1991 [TB, GD], daily at $1100-1800 \mathrm{~m}$ in Apr.-May 1992 [ATP, RF], at $1900 \mathrm{~m}$ on Mt. Nangkabulos in Mar. 1993 [JCTG, NADM], at $1555 \mathrm{~m}$ in Apr. 1999 [SEB, DHC], and regularly even in heavily disturbed secondary forests at 1300 $1620 \mathrm{~m}$ in 1993 and 1994 on the wide slopes north and south of the Lalawan River [AG]. Other sightings include birds seen daily 29 Apr.1 May 1983 (Table 11), 14 on 14-17 Mar. 1987 (Table 1j), in Dec. 1989-Jan. 1990 (Lambert, 1993), 27-28 Feb. 1992 (Table 1k), in Feb. 1993 (I. Gardner, in litt., 1993), Feb. 1994 (Table 1f), on 27 Feb. 1991 (Table 1m), in late Sep. 1994 (Table 1n), 17-18 Dec. 1994 (Table 1o), 4 Apr. 1996 (Table 1a), up to $1680 \mathrm{~m}$ in Dec. 1996 (Table 1b), at $1520 \mathrm{~m}$ on 7-8 Apr. 1998 (Table $1 \mathrm{~h}$ ), and singles at $1250 \mathrm{~m}$ on 2 days in Dec. 1998 (Table 1g).

\section{Lalage melanoleuca Black-and-white Triller}

The only confirmed record of the species from Kitanglad is of a pair collected at the mountain's base at Cabanglasan on 29 Oct. 1951 (ZMUC 759 60 ). A report of the species (Table $1 \mathrm{n}$ ) from Kitanglad suggests that this lowland Philippine endemic, normally restricted to elevations below 1000 m (Dickinson et al., 1991), may occasionally straggle up onto the mountain's higher slopes.

\section{Chloropsis flavipennis Philippine Leafbird}

VULNERABLE

This scarce endemic of the southern Philippines (Dickinson et al., 1991), down-listed to vulnerable (Collar et al., 1999) from endangered (Collar et al., 1994), is known from Kitanglad by 2 reports only: 2 specimens apparently collected at Malaybalay in Oct. 1951 (ZMUC; specimens not seen) and "6 Kitanglad Mts" noted without details from Feb. 1991 (Table 1m). It is a lowland species and may now have been extirpated by clearance of low-elevation forest in the Kitanglad region.

\section{Pycnonotus urostictus Yellow-wattled Bulbul}

The only record of this lowland species (Dickinson et al., 1991) from the Kitanglad area is of a bird collected by Salamonsen at Cabanglasan on 25 Oct. 1951 (ZMUC 799).

\section{Hypsipetes philippinus Philippine Bulbul}

This species was abundant at all elevations on Kitanglad. An Accipiter virgatus (FMNH 357393) was mist-netted on 17 Apr. 1993 on the 1100-m ridge carrying a fledgling of this species (FMNH 357477), and a recently fledged juvenile was sighted on the northwest slope on 28 Apr. 1994 [AG]. Two females collected Apr.-May 1999 at 1440-1620 m had yolking follicles [SEB, DHC]. Birds at high elevations $(1700-1850 \mathrm{~m})$ in May 1992 were quiet, in contrast to the populations at lower elevations [ATP, RF].

\section{Hypsipetes everetti Yellowish Bulbul}

Specimens were collected at Malaig and Cabanglasan at the base of Kitanglad in the early and middle twentieth century (Collar et al., 1999; ZMUC 23-35). Following the clearance of nearly all of the area's lowland forest, this lowland species (Dickinson et al., 1991) is presumably now absent from the Kitanglad area.

\section{Oriolus steerii Philippine Oriole}

Although numerous historical specimens of this species have been taken from Kitanglad (FMNH, PNM, YPM, ZMUC) (Ripley \& Rabor, 1961), no recent records exist. Considering its lowland affinities (Dickinson et al., 1991), it is possible that the species is now extirpated on the mountain.

\section{Irena cyanogaster Philippine Fairy-bluebird}

This species is known on Kitanglad only from sight records at $1100-1200 \mathrm{~m}$ on the west flank on 21 Apr.-1 May 1992 [ATP, RF].

\section{Parus elegans Elegant Tit}

Adults of this abundant species were attending an apparently active nest on 22 Apr. 1992 on a ridgetop at $1200 \mathrm{~m}, 2 \mathrm{~m}$ up in a rotting dead trunk ( $\sim 30$-cm diameter) [ATP]. The nest cavity was too deep to permit determination of the contents. Three fledged young were sighted on 27 Apr. 1997 near the Kalawaig River at $620 \mathrm{~m}$ [AG].

\section{Rhabdornis mystacalis Stripe-headed Rhabdornis}

This Philippine endemic is usually replaced above about $800 \mathrm{~m}$ by its congener, $R$. inornatus (Dickinson et al., 1991). Nevertheless, a handful of sight records places this species on the mountain: 27 Feb. 1992 (Table 1k), 2 on 14 Mar. 1990 (Table 1d), at $1100 \mathrm{~m}$ on 21 Apr. 1992 [ATP], at $1300-1550 \mathrm{~m}$ on the northeast slope on 31 Mar. and 5 Apr. 1994 [AG], at $670 \mathrm{~m}$ on the northwest slope on 26 Mar. 1993 and 21 Apr. 1994 [AG], and (at an oddly high elevation) at 
$1900 \mathrm{~m}$ on Mt. Nangkabulos in Mar. 1993 [JCTG, NADM]. Three historical specimens are from Cabanglasan at the mountain's base, collected by Salomonsen in Oct. 1951 (ZMUC 915-7). The seeming co-occurrence of this species with $R$. inomatus on Kitanglad suggests that their customary segregation (Kennedy et al., 2000) does not seem to operate at this site.

\section{Leonardina woodi Bagobo Babbler}

This secretive bird is evidently largely terrestrial: we mist-netted 1 individual and trapped 2 more (FMNH 357457-9) in snap-traps (coconut bait) at 1100-1500 m in Apr.-May 1992 and snap-trapped (earthworm bait) single birds at $1900 \mathrm{~m}$ and $2250 \mathrm{~m}$ on Mt. Nangkabulos in Mar.-Apr. 1993 (FMNH 364198-9). It was found at $1290-1340 \mathrm{~m}$ on the northeast slope in MarApr. 1994 and as low as $620 \mathrm{~m}$ on the northwest slope in the Kalawaig valley 29 Mar. 1993 [AG], well below its "usual" minimum elevation of $1000 \mathrm{~m}$ (Dickinson et al., 1991). Other records include several specimens from the $1960 \mathrm{~s}$ (SMF), 1 mist-netted on 12 Mar. 1990 (Table 1d), and a possible sighting on 10 Feb. 1994 (Table 1f). Collar et al. (1999) dropped this species from consideration as vulnerable, presumably on the grounds that its elusiveness and preference for high montane forest have led to its being underrecorded (Kennedy et al., 2000).

\section{Ptilocichla mindanensis Streaked Ground-babbler}

This species' maximum elevation is usually taken as $1000 \mathrm{~m}$ (Dickinson et al., 1991), so it is unsurprising that it is known from Kitanglad from 3 records only: a bird collected at Kaatoan at $1250 \mathrm{~m}$ on 11 Nov. 1951 by Salomonsen (ZMUC 105), others collected at the remarkably high elevations of $1500-1730 \mathrm{~m}$ in the 1960s (YPM 62398, SMF 46277-46278) (Ripley \& Rabor, 1961), and a sight record at $650 \mathrm{~m}$ on the northwest slope on 17 Apr. 1994 [AG]. Collar et al. (1999) dropped the species from consideration as near threatened, presumably because of its relative abundance wherever lowland limestone forest remains across Greater Mindanao (e.g., Brooks et al., 1996).

\section{Stachyris plateni Pygmy Babbler \\ NEAR THREATENED}

An uncommon endemic to Greater Mindanao. this species has been recorded up to about $1000 \mathrm{~m}$ on most of Mindanao's other mountain ranges (Dickinson et al., 1991). It is rare on Kitanglad, the only sightings being from 15 Mar.
1987, associated with Macronous striaticeps (Table 1j; Hornskov, 1995), and of birds at 590-750 $\mathrm{m}$ at the Kalawaig site in 1993-1994 [AG]. A single specimen was also collected at Cabanglasan at the foot of Kitanglad by Salomonsen on 27 Oct. 1951 (ZMUC 550) (Table 1c; Collar et al., 1999).

\section{Stachyris capitalis Rusty-capped Babbler}

The only Kitanglad records of this lowland species are of 3 collected at Cabanglasan at the mountain's base by Salomonsen on 25 Oct. 1951 (ZMUC 557-9).

\section{Micromacronus leytensis Miniature Tit-babbler} DATA DEFICIENT

This rare and poorly-known species is reportedly restricted to elevations below $1300 \mathrm{~m}$ on Greater Mindanao (Dickinson et al., 1991). Collar et al. (1999), however, provided reports at up to $1670 \mathrm{~m}$ and noted that most come from above $1000 \mathrm{~m}$. This species has only been reported once on Kitanglad, where A. Long saw 3 in canopy of montane forest with Phylloscopus trivirgatus in Jan. 1995 (Collar et al., 1999).

\section{Luscinia calliope Siberian Rubythroat}

A winter visitor to the northern Philippines (Dickinson et al., 1991); a Kitanglad sighting from late Sep. 1994 (Table 1n) was the first for Mindanao.

\section{Zoothera andromedae Sunda Ground-Thrush}

This species is known from Kitanglad from specimens collected in the 1960s (YPM 61808, 62397, SMF 46379-46380) in May and December (Ripley \& Rabor, 1961) and from 1 recent sighting at $1700 \mathrm{~m}$ on 14 Mar. 1987 (Table 1j).

\section{Turdus chrysolaus Brown-headed Thrush}

An uncommon winter visitor to the northern Philippines (Dickinson et al., 1991), a sighting of 2 on 13 Mar. 1987 was the first for Mindanao (Table 1j).

\section{Locustella fasciolata Gray's Grasshopper-warbler}

A rare winter visitor to the Philippines, known from elsewhere on Mindanao from only 2 specimens from Lanao del Norte and Misamis Oriental (FMNH 284066-7) (Dickinson et al., 1991). Hence, a 1960s specimen (SMF uncataloged) and a sighting at $950 \mathrm{~m}$ on the northeast slope of Kitanglad on 2 Jan. 1994 [AG] are notable.

\section{Orthotomus nigriceps Black-headed Tailorbird}

A single specimen was collected at Cabanglasan at the base of Kitanglad on 21 Oct. 1951 
(ZMUC 370) (Collar et al., 1999), by Salomonsen, but no recent records exist, and this lowland Greater Mindanao endemic (Dickinson et al., 1991 ) is presumably now extirpated in the area.

\section{Bradypterus caudatus Long-tailed Ground-warbler}

Evidently chiefly terrestrial, this species was mist-netted and snap-trapped (coconut bait) twice in May 1992 at $1500-1800 \mathrm{~m}$ (FMNH 357480-357481), in Apr.-May 1999 at $1455 \mathrm{~m}$ [SEB, DHC], and snap-trapped (earthworm bait; FMNH 364200) at $1900 \mathrm{~m}$ on Mt. Nangkabulos in Mar. 1993. Other Kitanglad records include specimens from the 1960s (YPM 61815-61816, SMF 48166) (Ripley \& Rabor, 1961) and numerous birds seen and heard as follows: " $1-16$ noted almost daily" in Mar. 1987 (Table 1j), on 5 Oct. 1991 [TB, GD], daily 26-29 Feb. 1992 (Table 1k), in Feb. 1993 (I. Gardner, in litt., 1993), 1 at $630 \mathrm{~m}$-an extremely low elevation for this species, which is normally only found above $1000 \mathrm{~m}$ (Dickinson et al., 1991)-and 1 at $1120 \mathrm{~m}$ on the northwest slope on 14 Apr. 1993 [AG], on 9-10 Feb. 1994 (Table 1f), in Dec. 1994 and Jan. 1995 (Table 1o), a probable sighting in Apr. 1996 (Table 1a), and birds heard only in Dec. 1996 (Table 1b). This frequency of records, especially considering the species' elusive habits, support the decision of Collar et al. (1999) to drop it from consideration as near threatened.

\section{Rhinomyias goodfellowi Goodfellow's Jungle- flycatcher \\ NEAR THREATENED}

This Mindanao endemic is shy and retiring and hence is known from Kitanglad principally from specimens (FMNH 262539, YPM 6192061921, 62463, SMF 46284) (Ripley \& Rabor, 1961) and only a handful of recent records: 11 collected in Apr.-May 1992 (FMNH $357497-$ 357507 ) by ATP, including 2 juvenile-plumaged individuals on 13 May at $1700 \mathrm{~m}$, and sightings in Jan. 1992 (R. J. Timmins, pers. comm., 1992), at $1290-1620 \mathrm{~m}$ on the northeast slope in 1993 and at $750 \mathrm{~m}$ on 26 Mar. $1993 \mathrm{~m}$ in primary forest in the upper Kalawaig valley [AG]. The $750-\mathrm{m}$ sighting is an unusually low elevation for the species (usually above $1000 \mathrm{~m}$; Dickinson et al., 1991). Collar et al. (1999) down-listed the species to near threatened from vulnerable, presumably because of its relative abundance in undisturbed montane forest.

\section{Ficedula mugimaki Mugimaki Flycatcher}

A rare winter visitor to the Philippines (Dickinson et al., 1991), with the 5 Mindanao records all from Kitanglad: a female collected (apparently no longer in YPM) on 22 Dec. 1960 (Ripley \& Rabor, 1961), a female seen on 15 Mar. 1987 (Table 1j), 1 in late Sep. 1994 (Table 1n), a male in Dec. 1996 (Table 1b), and a male at $1350 \mathrm{~m}$ on 3 Dec. 1998 (Table $1 \mathrm{~g}$ ).

\section{Rhipidura superciliaris Blue Fantail}

This species is known from Kitanglad only from our records: a specimen (FMNH 357536) from $1100 \mathrm{~m}$ and sight records from 1100 to 1800 in Apr.-May 1992 [ATP, RF], 1 bird at $1180 \mathrm{~m}$ on the northeast slope on the Lalawan valley on 16 Jan. 1994, and regular sightings at $600-840 \mathrm{~m}$ on the northwest slope in 1993 and 1994 in mature secondary growth, occasionally in mixed flocks [AG]. The records from $1800 \mathrm{~m}$ are quite exceptional for a species that has not previously been recorded above $1200 \mathrm{~m}$ (Dickinson et al., 1991); however, the species was not observed regularly and may have been represented by strays from the adjacent lowlands only.

\section{Motacilla alba White Wagtail}

This species is a rare winter visitor to the Philippines, not previously known from Mindanao (Dickinson et al., 1991). Sightings on 20 and 25 Apr. 1994 at $660-700 \mathrm{~m}$ on the northwest slope are the first for the island $[\mathrm{AG}]$.

\section{Anthus hodgsoni Olive Tree-pipit}

Another uncommon winter resident (Dickinson et al., 1991) for which few Kitanglad records exist. Rabor collected males on 24 Mar. and $22 \mathrm{Dec}$. 1960 and females on 6 Apr. and 20 Dec. 1960 (YPM 61741-61742, 62358-62359), and more recently Jensen and Hornskov (Table 1j) sighted 1-5 "almost daily" 13-17 Mar. 1987, Simpson (Table 1o) sighted 1 on 15 Dec. and 2 on 17 Dec. 1994, and singles were observed on 14 and 17 Jan. 1994 at $1310-1320 \mathrm{~m}$ on the northeast slope [AG].

\section{Anthus gustavi Pechora Pipit}

An uncommon winter visitor to the Philippines (Dickinson et al., 1991) but possibly overlooked in the field, as most records from Kitanglad have come from mist-nets: Rabor collected a male on 26 Mar. and a female on 22 Apr. 1960 (YPM 61743-6174); another was mistnetted (FMNH 357446) on the west slope in Apr. 1992 [ATP, RF]. A long series of specimens in SMF (e.g., SMF 47195) further indicates that its frequency may have been underappreciated. Observations include on the northeast slope at $1280 \mathrm{~m}$ on 1 Apr. 1994 [AG], 29 Feb. 1992 
(Table $1 \mathrm{k}$ ), and 4 seen at $1500-1600 \mathrm{~m}$ on $9 \mathrm{Apr}$. 1998 (Table 1h).

\section{Lanius validirostris Mountain Shrike \\ NEAR THREATENED}

A pair of adults was observed feeding 2 recently fledged young (tail about $60 \%$ of adult length) at $1750 \mathrm{~m}$ on 7 May 1992 [ATP, RF]. The juveniles had plumage similar to that of the adults, though washed with gray on the entire underparts instead of only on the flanks, and with a short, orange bill. A bird was mist-netted (FMNH 357448) at $1800 \mathrm{~m}$ in May 1992, and other individuals were found at $1900-2250 \mathrm{~m}$ on Mt. Nangkabulos in Mar.-Apr. 1993 (FMNH 364185-364186), a pair at $1420-1460 \mathrm{~m}$ on the northeast slope in Apr. 1993, and a single individual on 1 Apr. 1994 at 1510 m in dry degraded habitat [AG]. In the northwest, it was seen at extremely low elevations (records cited in Dickinson et al., 1991) for this species (720$1030 \mathrm{~m}$ ) in Apr. 1994 [AG]. Other records include 1960s specimens (PNM 10187, YPM 61786-61787, SMF various) (Ripley \& Rabor, 1961) and sight records on 15 and 17 Mar. 1987 (Table 1j), 12-14 Mar. 1990 (Table 1d), 2 seen by R. Hopf in Feb. 1994 (Table 1f), on 4 Apr. 1996 (Table la), in Dec. 1996 (Table 1b), at 1400-1600 in Apr. 1998 (Table 1h), and at $1450 \mathrm{~m}$ on 9 Dec. 1998 (Table $1 \mathrm{~g}$ ).

\section{Basilornis miranda Apo Myna}

\section{NEAR THREATENED}

Our records of $B$. mirandus include a single group at $1300 \mathrm{~m}$ in Apr. 1992, with more birds and 3 mist-netted (FMNH 357664-357665, 395835) at $1500-1800 \mathrm{~m}$ in May 1992, 2 mist-netted at $1900 \mathrm{~m}$ and more sighted up to $2250 \mathrm{~m}$ on Mt. Nangkabulos in Mar.-Apr. 1993 [JCTG, NADM], and regular sightings, often in connection with natural clearings (wind- and firedamaged areas), at $1380-1860 \mathrm{~m}$ on the northeast slope in 1993 and 1994, on the northwest slope on 28 Mar. 1993 at 1090 m, and on 21 Apr. 1994 at $1150 \mathrm{~m}$ [AG]. It was invariably found in flocks of 2-8 individuals in which as many as 4 were in what appeared to be adult plumage, suggesting that the species may be a cooperative breeder. Other sightings include birds seen in late Apr. 1983 (Table 11), 2 on 14 Mar., 41 in a single flock on 15 Mar., l on 16 Mar. and 14 on 17 Mar. 1987 (Table 1j), 2 on 15 Mar. 1990 (Table 1d), on 27 Feb. 1991 (Table 1m), 2 in Aug. 1991 (G. Anderson pers. comm., 1991), on 11 Feb. 1994 (Table 1f), in late Sep. 1994 (Table 1n), 1 on 15 Dec. 1994 (Table 1o), on 3 Apr. 1996 (Table 1a), 12 at $\sim 1500 \mathrm{~m}$ and 6 at $1800 \mathrm{~m}$ in Dec. 1996 (Table 1b), 6 groups of up to 13 birds at $1520-1700 \mathrm{~m}$ on 5-8 Apr. 1998 (Table $1 \mathrm{~h}$ ), and groups of up to 6 at $1520 \mathrm{~m}$ most days 2-10 Dec. 1998 (Table lg). Numerous specimens place the species on the mountain (FMNH, PNM, YPM, KUNHM, SMF) (Ripley \& Rabor, 1961).

\section{Nectarinia sperata Purple-throated Sunbird}

The only records of this lowland species are 3 specimens: 2 collected at the base of Kitanglad at Cabanglasan on 19 Oct. 1951 by Salomonsen (ZMUC 490-1), and 1 at Bo Kaatuan in 1964-1965 (SMF 46663).

\section{Aethopyga primigenius Grey-hooded Sunbird \\ NEAR THREATENED}

This Mindanao montane endemic species is common on Kitanglad, demonstrated by numerous specimens and sight records. It is considered near threatened as a precaution given its small extent of occurrence.

\section{Aethopyga pulcherrima Metallic-winged Sunbird}

The only records of this species from the Kitanglad area are of 5 specimens collected at Cabanglasan at the mountain's base in Oct. 1951 by Salamonsen (ZMUC 1121-1125).

\section{Aethopyga boltoni Apo Sunbird}

NEAR THREATENED

Numerous specimens of this species exist from the 1950s and 1960s (ZMUC, FMNH, SMF, YPM) (Ripley \& Rabor, 1961). We observed birds at $1500-1800 \mathrm{~m}$ in May 1992, including 3 mistnetted (FMNH 357630-357633) and a further individual sighted [ATP, RF], and at 1900$2250 \mathrm{~m}$ on Mt. Nangkabulos in Mar.-Apr. 1993, including 2 birds mist-netted at $1900 \mathrm{~m}$ and 15 at $2250 \mathrm{~m}$ [JCTG, NADM]. On the northeast slope, it was observed during both 1993 and 1994 [AG]. A male and a pair at 590$600 \mathrm{~m}$ near the Kalawaig River on 29 Mar. 1993 and 26 Apr. 1994 [AG] represent an extremely low elevation for this species, which is normally found only above $1500 \mathrm{~m}$ (Dickinson et al., 1991). Three other sightings include 1 on 4 Apr. 1996 (Table 1a), a male at $1735 \mathrm{~m}$ in Dec. 1996 (Table 1b), and 2 males on 7 Apr. 1998 at 1650 $1700 \mathrm{~m}$ (Table 1h).

\section{Aethopyga shelleyi Lovely Sunbird}

This species is apparently very scarce on Kitanglad, as no specimens exist, and the only sight records are of $1-3$ on 14,15 , and 17 Mar. 
1987 (Table 1i), and 1 seen at $1800 \mathrm{~m}$ in May 1992 [ATP].

\section{Arachnothera clarae Naked-faced Spiderhunter}

Although known from a number of Kitanglad specimens collected in the $1960 \mathrm{~s}$ at $1400-1500 \mathrm{~m}$ (FMNH, PNM, YPM, SMF) (Ripley \& Rabor, 1961), rather few recent records place this species on the mountain. We found it at $1100-1200 \mathrm{~m}$ in Apr. 1992 [ATP, RF], at $1900 \mathrm{~m}$-well above the maximum elevation of $1300 \mathrm{~m}$ given by Dickinson et al. (1991) - on Mt. Nangkabulos in Mar. 1993 [JCTG, NADM], and at $1180 \mathrm{~m}$ on the northeast slope on 14 Mar. 1993 and $630-1050 \mathrm{~m}$ on the northwest slope in 1993 and 1994 [AG], but the only other recent sighting is from Dec. 1996 (Table 1b). Collar et al. (1999) down-listed the species from near threatened considering its broad habitat tolerances and distribution across the eastern Philippines.

\section{Arachnothera longirostris Little Spiderhunter}

The only confirmed records of this species from the mountain are of 6 collected at Cabanglasan at Kitanglad's foot by Salomonsen in Oct. 1951 (ZMUC 48-53) and several from Bo Kaatuan (1500 m, SMF 46636-46671). These relatively high-elevation occurrences lend some credence to reports of this species, normally restricted to below $1000 \mathrm{~m}$ (Dickinson et al., 1991), from $1900 \mathrm{~m}$ on Mt. Nangkabulos in Mar. 1993 (Table 1c) and from 17 Mar. 1987 (Table 1i).

\section{Prionichilus olivaceus Olive-backed Flowerpecker}

Our records at $900-1100 \mathrm{~m}$ on the west slope in Apr. 1992 (FMNH 357587-357590) [ATP, RF] and from $650 \mathrm{~m}$ (an adult with fledgling) on 22 Apr. 1994 and $810 \mathrm{~m}$ on 26 Apr. 1994 on the northwest slope [AG] are the only records of this species from Kitanglad except for a single bird collected at Cabanglasan at the mountain's base on 22 Oct. 1951 (ZMUC 500) and several from $1500 \mathrm{~m}$ at Bo Kaatuan (e.g., SMF 46579).

\section{Dicaeum aeruginosum Striped Flowerpecker}

The only report of this species from Kitanglad is of 1 seen on 9 Feb. 1994 (Table 1f). This widespread species is not unexpected in northern Mindanao, and as such we accept it provisionally as reasonable.

\section{Dicaeum anthonyi Flame-crowned Flowerpecker NEAR THREATENED}

This species occurs at quite low densities on Kitanglad, found in particular at higher eleva- tions and seeming quite unpredictable in its occurrence. Records include 1 on 5 Oct. 1991 [TB, GD]; 2 mist-netted at $1100 \mathrm{~m}$ and 1 seen in the field at $1200 \mathrm{~m}$ in Apr. 1992 and 6 at $1500 \mathrm{~m}$ in May 1992 (FMNH 357599-357605) [ATP, RF]; and at $1900 \mathrm{~m}$ on Mt. Nangkabulos in Mar. 1993 [JCTG, NADM]. Other recent sightings include birds seen in late Apr. 1983 (Table 11), in Feb. 1991 (Table 1m), on 28 Feb. 1992 (Table 1k), a male seen by R. Hopf on 10 Feb. 1994 (Table 1f), an adult male and presumed immature male seen on 10 Jan. 1995 (Table 1o), 1 seen by D. Cooper in Apr. 1996 (Table 1a), and 1 at $1300 \mathrm{~m}$ on 4 Dec. and a pair at $1250 \mathrm{~m}$ on 6 Dec. 1998 (Table 1g). Numerous old specimens (FMNH, YPM, ZMUC, SMF) (Salomonsen, 1953; Ripley \& Rabor, 1961) also document its presence.

\section{Dicaeum proprium Whiskered Flowerpecker \\ NEAR THREATENED}

This uncommon species was the last montane Mindanao endemic to be recorded from Kitanglad, reported for the first time in the broader scientific literature in this paper. It has recently been down-listed from vulnerable (Collar et al., 1994) to near threatened (Collar et al., 1999). Sightings with good details are of 6-10 birds at $1500 \mathrm{~m}$ in Apr. 1993 (Table 1c) and on 11 Jan. 1994 at $1300 \mathrm{~m}$ on the northeast slope [AG].

\section{Dicaeum pygmaeum Pygmy Flowerpecker}

This species is scarce on Kitanglad, known from only 3 specimens from 9 Dec. 1951 at Kibangay at $1200 \mathrm{~m}$ (ZMUC 334) and from 2 and 12 May 1960 (YPM 60238-60239) (Ripley \& Rabor, 1961) and sight records in Feb. 1993 (I. Gardner, in litt., 1993) and at $1250 \mathrm{~m}$ and $1300 \mathrm{~m}$ (pairs) in Dec. 1998 (Table 1g).

\section{Zosterops everetti Everett's White-eye}

Restricted to lowlands below 1000 m(Dickinson et al., 1991), $Z$. everetti replaces the abundant Mountain White-eye $Z$. montanus on Kitanglad's lower flanks. It was sighted almost daily between 420 and $800 \mathrm{~m}$ on the northwest slope, with up to 10 individuals per flock, and 5 were seen at $1170 \mathrm{~m}$ on 7 Apr. 1993 [AG], but otherwise the only records are sightings of 3-40 seen 14-17 Mar. 1987 (Table 1j) and up to 8 on 3 days in Mar. 1990 (Table 1d) and 1 on 26 Feb. 1991 (Table 1m).

\section{Erythrura coloria Red-eared Parrotfinch} NEAR THREATENED

This species is common on Kitanglad, and we sighted at least 2 on 5-6 Oct. 1991 [TB, GD], 
several at $2250 \mathrm{~m}$ on Mt. Nangkabulos in Mar.-Apr. 1993 including 1 mist-netted (FMNH 364255), 4 at 1440-1525 $\mathrm{m}$ in Apr.-May 1999 [SEB, DHC], and 1 at $1300-1420 \mathrm{~m}$ on the northeast slope in 1993-1994 [AG]. Other recent sightings include 3 on 15 Mar. 1987 (Table 1j), 2 at 1450-1500 m in early Jan. 1990 (Lambert, 1993), several in Feb. 1991 (Table $1 \mathrm{~m}$ ), birds seen daily 27-29 Feb. 1992 (Table 1k), daily on 8-10 Feb. 1994 (Table 1f), 1 in late Sep. 1994 (Table 1n), 6 seen (by A. Long) on 10 Jan. and 2 on 11 Jan. 1995 (Table 1o), 1 on 5 Apr. 1996 (Table 1a), and 5 at $1250-1400 \mathrm{~m}$ in Dec. 1996 (Table 1b). Numerous old specimens of the species are from 1400 to $1500 \mathrm{~m}$ (FMNH, YPM, SMF, and others) (Ripley \& Rabor, 1961). The species' relative abundance on the mountain supports the decision of Collar et al. (1999) to down-list it to near threatened from vulnerable; depending on its status elsewhere in Mindanao, it may in fact be better treated as least concern.

\section{Lonchura punctulata Scaly-breasted Munia}

A sighting of 3 birds at $1050 \mathrm{~m}$ in fallow land on the northeast slope on 18 Jan. 1994 [AG] becomes the earliest known record of this species in Mindanao, it having been first recorded on the island only recently (Brooks \& Dutson, 1997).

\section{Serinus estherae Mountain Serin}

This species presents an interesting distributional quandary, known only from Indonesia (Sumatra, Java, Sulawesi) and the mountains of Mindanao. It was long thought to be known on Kitanglad only by a single specimen from 19 Apr. 1960 (YPM 58898) (Ripley \& Rabor, 1961) and from a sighting at $1900 \mathrm{~m}$ on Mt. Nangkabulos in Mar. 1993 [JCTG, NADM]; however, an important series collected in 1965 (SMF 4639946404,1 uncataloged) documents its presence with greater clarity. Detailed comparisons of this specimen material are required to clarify the taxonomic status of these populations.

\section{Pyrrhula leucogenys White-cheeked Bullfinch}

Relatively common at high elevations on Kitanglad, we recorded and collected this species at $1500-1800 \mathrm{~m}$ in May 1992 (FMNH 357659 357660) [ATP, JCTG, NADM], mist-netted singles at both $1900 \mathrm{~m}$ and $2250 \mathrm{~m}$ on $\mathrm{Mt}$. Nangkabulos in Mar.-Apr. 1993 (FMNH 364253364254) [JCTG, NADM], and found single birds or flocks of up to 5 individuals at $1680-1810 \mathrm{~m}$ on the northeast slope from primary to degraded secondary forest in 1993-1994 [AG]. Other recent sightings include $7-16$ on $14-17$ Mar. 1987 (Table 1j), 13 on 13-15 Mar. 1990 (Table 1d), 1 on 27 Feb. 1991 (Table 1m), a pair on 8 Feb. 1994 (Table 1f), 1 heard in late Sep. 1994 (Table 1n), 4 on 11 Jan. 1995 (Table 1o), sightings on 3-4 Apr. 1996 (Table la), and several at $\sim 1500 \mathrm{~m}$ in Dec. 1996 (Table 1b). Numerous specimens place this species on the mountain from the 1950s and 1960s (FMNH, PNM, YPM, ZMUC, SMF) (Ripley \& Rabor, 1961).

\section{Hypothetical Records}

\section{Aviceda jerdoni Jerdon's Baza}

An uncommon resident on the larger islands throughout the Philippines (Dickinson et al, 1991), this species was reported from Kitanglad on 26 Feb. 1991 (Table 1m). Given its rarity, we prefer to treat this sighting as hypothetical, although it is supported by the fact that a female was collected (ZMUC) on 20 Nov. 1952 from the Pulangi River, Bukidnon, which rises on Kitanglad (Parkes, 1961). Collar et al. (1999) dropped the species from consideration as near threatened, presumably because of its huge range.

\section{Butastur liventer Rufous-winged Buzzard}

A bird seen on 28 Feb. 1992 and tentatively identified as $B$. liventer (Table $1 \mathrm{k}$ ), which is not known from the Philippines (Dickinson et al., 1991), was likely to have in fact been $B$. indicus (B. King, in litt., 1996), which is a relatively common winter visitor to Kitanglad.

\section{Cacomantis merulinus Plantive Cuckoo}

With the reidentification of the voucher specimen mist-netted on 25 Apr. 1992 at $1100 \mathrm{~m}$ (FMNH 357424) as C. sepulcralis [ATP], it seems sensible to consider the only other sightings of the species from Kitanglad, from 1900 to $2250 \mathrm{~m}$ on Mt. Nangkabulos in Mar.Apr. 1993 [JCTG, NADM] and on 1 May 1983 (Table 11), as hypothetical.

\section{Bubo philippensis Philippine Eagle-owl}

VULNERABLE

This species of deep forest on the larger Philippine islands, reportedly at low elevations (Dickinson et al., 1991) was recently down-listed to vulnerable (Collar et al., 1999) from endangered. The only 2 Kitanglad reports involve 1 heard on 19 Mar. 1987 by Jensen and Hornskov (Table 1j), who had some prior experience with 
the species' vocalizations, and a molted secondary feather found in mature secondary forest at $730 \mathrm{~m}$ on the northwest slope on $27 \mathrm{Apr} .1994$ [AG]. It is very surprising that convincing records of this species have not been obtained, given the amount of nocturnal effort that other observers have carried out on Kitanglad, so we feel that we have to consider these records hypothetical until more conclusive documentation is obtained.

\section{Collocalia whiteheadi Whitehead's Swiftlet DATA DEFICIENT}

This species is a Philippine montane endemic known with certainty from very few specimens (Dickinson et al., 1991; Kennedy et al., 2000). It has been reported at $2250 \mathrm{~m}$ on Mt. Nangkabulos in Mar.-Apr. 1993 [JCTG, NADM], in Feb. 1994 when Hornbuckle (Table if) noted that "two apparently larger birds high on Kitanglad when the cloud was low could have been the rare Whitehead's Mountain Swiftlet C. whiteheadi," at $1450-1700 \mathrm{~m}$ on the northeast slope in 1993 and 1994 [AG], and in Sep. 1994 (Table 1n). Given its rarity and the lack of specimen documentation, however, acceptance of this species as occurring on Kitanglad should await full documentation. The lack of information regarding this species led Collar et al. (1999) to consider it data deficient rather than vulnerable (Collar et al., 1994).

\section{Apus pacificus Fork-tailed Swift}

An uncommon passage migrant in the Philippines, not known south of Negros (Dickinson et al., 1991). A possible sighting of the species on 2 Jan. 1994 at $500 \mathrm{~m}$ on the northeast slope of Kitanglad [AG] would represent the first record for Mindanao; however, given the potential for confusion between this species and A. affinis, which is also not known from Mindanao but which is expanding in the Philippines (Evans et al., 1993), we feel that it is best to treat this record as unconfirmed.

\section{Mirafia javanensis Singing Bushlark}

This species is likely to occur around the Kitanglad massif but has been reported only once, in late Sep. 1994 (Table 1n), so we consider it hypothetical.

\section{Alauda gulgula Oriental Skylark}

A sighting from $960 \mathrm{~m}$ on the eastern slopes of Kitanglad [AG], which would represent only the second Mindanao record (Meyer de Schauensee,
1957), is considered hypothetical without better documentation of the identification.

\section{Ficedula basilanica Little Slaty Flycatcher}

VULNERABLE

This rare forest endemic, restricted to below $1000 \mathrm{~m}$ on Greater Mindanao (Dickinson et al., 1991), has not been recorded with certainty on Kitanglad: for lack of better detail or voucher specimens, we retracted our [TB, GD] sightings from $1200 \mathrm{~m}$ on 5 Oct. 1991 ("two birds were found in kaingin at the edge of forest in a mixedspecies foraging flock"; Evans et al., 1993) and consider as hypothetical those by R. Hopf from $1200 \mathrm{~m}$ on 9 Feb. 1994 (Table 1f), from late Sep. 1994 when apparently "quite common" (Table $1 \mathrm{n}$ ), and from $1300 \mathrm{~m}$ on 3 Dec. 1998 (Table $1 \mathrm{~g}$ ).

\section{Erythrura viridifacies Green-faced Parrotfinch \\ VULNERABLE}

Recent reports of parrotfinches resembling $E$. viridifacies from Kitanglad and elsewhere on Mindanao are of great interest, in particular 4 specimens collected on Mt. Hilong-Hilong in May 1971 (SUNSM) and 6 on Kitanglad in 1996 (PNM) (Nordeco \& Denr, 1998). Dickinson et al. (1991) considered the species endemic to Luzon and Negros, but birds were recently found on Panay (Curio, 1997). Considering its irruptive habits, it is not inconceivable that birds could have escaped detection on (or recently colonized) Mindanao. However, criteria for separation of $E$. viridifacies from immature $E$. coloria are unclear, and D. Allen and F. Danielsen (in litt., 1999), who have examined the Kitanglad specimens, believe them more likely to be E. coloria.

\section{Analysis}

We have presented a summary of the known avifauna of the Kitanglad massif on Mindanao Island, Philippines, including 198 species known from the massif (Tables 2 and 3 ) and another 10 that are considered hypothetical or not accepted from the region. The records cover about 6 decades, including specimen series assembled by professional collectors, detailed altitudinal surveys by professional ornithologists, and observations by bird-watchers. Although the data that serve as the foundation of this monograph are thus quite heterogeneous (Heaney, 2002; Heaney et al., 2002), in this section we attempt to 
synthesize aspects of the avifauna of the Kitanglad region.

Mist-Net Capture Rates-Knowledge of patterns of abundance and rarity in a local fauna can be critical to understanding a wide variety of biological phenomena, including community structure and interactions, autecological requirements of species, and regional patterns of diversity and endemism. Nevertheless, estimation of abundance is an extremely challenging task. Species differ not only in abundance but also in detectability, and the 2 phenomena are easily confounded. In particular, caution must be used when comparing relative abundances between mist-net samples, because mist-nets sample canopy (e.g., Coracina mcgregori) and terrestrial (e.g., Leonardina woodi) species poorly; moreover, mist-nets sample species differentially depending on distances moved by individuals (Remsen \& Good, 1996).

Patterns of abundance as represented in mistnet captures were contrasted between the 2 elevations for which detailed data were available: $1100-1300 \mathrm{~m}$ and $1500-1800 \mathrm{~m}$ (Table 4). Thirty species were detected at $1100-1300 \mathrm{~m}$, whereas 35 species were detected at $1500-1800 \mathrm{~m}$; these differences probably reflected the overall canopy height differences between the 2 habitats, although differences in sampling effort should also be considered. The canopy at higher elevations was sufficiently low for more species to become nettable, accounting for the greater species diversity at higher elevations.

At $1100-1300 \mathrm{~m}, 4$ species reached relative abundances of more than 40 individuals per 1000 net-hours (Table 4): Ficedula hyperythra (144.36), Hypsipetes philippinus (102.26), Rhipidura nigrocinnamomea (96.24), and Parus elegans (48.12). At 1500-1800 m, 6 species were detected at more than 40 individuals per 1000 net-hours: Pachycephala philippensis (75.58), Ficedula hyperythra (72.67), Hypocryptadius cinnamomeus and Rhipidura nigrocinnamomea (58.14 each), Turdus poliocephalus (46.51), and Hypsipetes philippensis (40.70). Interestingly, none of these high-elevation species reached much more than half the densities of $F$. hyperythra at the lower elevations. Although preliminary in nature, this pattern suggests that abundances may be more evenly distributed at higher elevations, with fewer dominant species and more relatively common species, at least within the understory community.
Overall capture rates at these elevational stations were 697-770 individuals per 1000 netdays. These capture rates contrast strongly with those for other islands in the Philippines: on Mindoro and Negros in 1991, overall capture rate were $\sim 1430$ individuals per 1000 net-days (Evans et al., 1993). These differences may result from distinct styles of setting nets (e.g., many of the Field Museum nets were set for bats, which means that they were higher, more open, and along ridgetops; our experience is that such "bat" nets yield significantly fewer birds than do "bird" nets). Differences in capture rates could also result from different forest structure, avian community structure, or seasons of visit.

Flock Membership-Mixed-species flocks accounted for a considerable portion of the birds observed during our studies on Mt. Kitanglad (summary in Table 5). Although these flocks were often the only birds apparent in the forest, their composition varied considerably between the lower forest ridges $(1100-1300 \mathrm{~m})$ and the montane forest $(1500-1700 \mathrm{~m})$. On the lower ridges, flocks were dominated by Rhipidura nigrocinnamomea, Aethopyga primigenius, Parus elegans, Coracina mcgregori, Dicaeum bicolor, and D. hypoleucum, all of which were present in $>69 \%$ of the 15 flocks observed. Twenty-three species were observed in flocks (3-11 observed in individual flocks, average 8.0 species per flock), amounting for almost half the avifauna recorded at $1100-1300 \mathrm{~m}$.

At higher elevations, flock structure changed noticeably. More bird activity was observed outside the flocks. Only Zosterops montanus approached ubiquity (70\% of 10 flocks), although many species were present in $40-60 \%$ of flocks. Again, flocks were diverse, with 20 species observed participating and 2-11 (average 7.1) species per flock observed. An unexpected member of 2 flocks at this elevation was Collocalia esculenta, several individuals of which were observed to focus their foraging activities inside the forest where the flock was located and even to glean insects directly from leaves of trees. Many flocks on the higher-elevation ridges would better be characterized as family groups of single species, with a few individuals of other species associated.

Features of the Elevational TransectElevational transect studies of faunas have much to offer toward understanding ecological and 
TABLE 4. Summary of number of individuals captured and individuals per 1000 net-hours captured at 2 elevational levels on Mt. Kitanglad by the 1992-1993 FMnH groups. Total net-days $=174(1100-1300 \mathrm{~m})$ and 365 $(1500-1800 \mathrm{~m})$.

\begin{tabular}{|c|c|c|c|c|}
\hline \multirow[b]{2}{*}{ Species } & \multicolumn{2}{|c|}{ Total individuals } & \multicolumn{2}{|c|}{ Individuals per 1000 hours } \\
\hline & $1100-1300 \mathrm{~m}$ & $1500-1800 \mathrm{~m}$ & $1100-1300 \mathrm{~m}$ & $1500-1800 \mathrm{~m}$ \\
\hline \multicolumn{5}{|l|}{ Family Accipitridae } \\
\hline Accipiter trivirgatus & 1 & 0 & 6.0 & 0.0 \\
\hline A. virgatus & 0 & 2 & 0.0 & 5.8 \\
\hline \multicolumn{5}{|l|}{ Family Falconidae } \\
\hline Microhierax erythrogonys & 0 & 1 & 0.0 & 2.9 \\
\hline \multicolumn{5}{|l|}{ Family Columbidae } \\
\hline Chalcophaps indica & 2 & 1 & 12.0 & 2.9 \\
\hline Phapitreron spp. & 3 & 8 & 18.0 & 23.3 \\
\hline Ptilinopus occipitalis & 1 & 1 & 6.0 & 2.9 \\
\hline \multicolumn{5}{|l|}{ Family Psittacidae } \\
\hline Prioniturus discurus & 0 & 6 & 0.0 & 17.4 \\
\hline \multicolumn{5}{|l|}{ Family Cuculidae } \\
\hline Cacomantis sepulcralis & 2 & 2 & 12.0 & 5.8 \\
\hline Cuculus fugax & 0 & 3 & 0.0 & 8.7 \\
\hline C. saturatus & 1 & 1 & 6.0 & 2.9 \\
\hline \multicolumn{5}{|l|}{ Family Strigidae } \\
\hline Otus megalotis & 1 & 0 & 6.0 & 0.0 \\
\hline O. mirus & 0 & 4 & 0.0 & 11.6 \\
\hline \multicolumn{5}{|l|}{ Family Caprimulgidae } \\
\hline Caprimulgus manillensis & 0 & 1 & 0.0 & 2.9 \\
\hline \multicolumn{5}{|l|}{ Family Picidae } \\
\hline Chrysocolaptes lucidus & 1 & 1 & 6.0 & 2.9 \\
\hline \multicolumn{5}{|l|}{ Family Pycnonotidae } \\
\hline \multirow{2}{*}{\multicolumn{5}{|c|}{ Family Paridae }} \\
\hline & & & & \\
\hline Parus elegans & 8 & 4 & 48.1 & 11.6 \\
\hline \multicolumn{5}{|l|}{ Family Sittidae } \\
\hline Sitta oenochlamys & 1 & 9 & 6.0 & 26.2 \\
\hline \multicolumn{5}{|l|}{ Family Rhabdornithidae } \\
\hline Rhabdornis inornatus & 1 & 0 & 6.0 & 0.0 \\
\hline \multicolumn{5}{|l|}{ Family Timaliidae } \\
\hline Leonardina woodi & 1 & 0 & 6.0 & 0.0 \\
\hline Macronous striaticeps & 5 & 3 & 30.1 & 8.7 \\
\hline \multicolumn{5}{|l|}{ Family Turdidae } \\
\hline Turdus poliocephalus & 0 & 16 & 0.0 & 46.5 \\
\hline Family Sylvidae & & & & \\
\hline Orthotomus heterolaemus & 0 & 5 & 0.0 & 14.5 \\
\hline Phylloscopus olivaceus & 6 & 2 & 36.1 & 5.8 \\
\hline Family Muscicapidae & & & & \\
\hline Eumyias panayensis & 3 & 1 & 18.0 & 2.9 \\
\hline Ficedula hyperythra & 24 & 25 & 144.4 & 72.7 \\
\hline F. westermanni & 4 & 2 & 24.1 & 5.8 \\
\hline Rhinomyias goodfellowi & 6 & 7 & 36.1 & 20.4 \\
\hline Rhipidura nigrocinnamomea & 16 & 20 & 96.2 & 58.1 \\
\hline R. superciliaris & 2 & 0 & 12.0 & 0.0 \\
\hline Family Pachycephalidae & & & & \\
\hline Pachycephala philippinensis & 4 & 26 & 24.1 & 75.6 \\
\hline Family Motacillidae & & & & \\
\hline Anthus gustavi & 1 & 0 & 6.0 & 0.0 \\
\hline Family Laniidae & & & & \\
\hline Lanius validirostris & 0 & 1 & 0.0 & 2.9 \\
\hline Family Sturnidae & & & & \\
\hline Basilornis miranda & 0 & 3 & 0.0 & 8.7 \\
\hline Family Nectarinidae & & & & \\
\hline Aethopyga boltoni & 0 & 3 & 0.0 & 8.7 \\
\hline A. primigenius & 1 & 8 & 6.0 & 23.3 \\
\hline Family Dicaedae & & & & \\
\hline
\end{tabular}


TABLE 4. Contimed.

\begin{tabular}{|c|c|c|c|c|}
\hline \multirow[b]{2}{*}{ Species } & \multicolumn{2}{|c|}{ Total individuals } & \multicolumn{2}{|c|}{ Individuals per 1000 hours } \\
\hline & $1100-1300 \mathrm{~m}$ & $1500-1800 \mathrm{~m}$ & $1100-1300 \mathrm{~m}$ & $1500-1800 \mathrm{~m}$ \\
\hline Dicaeum spp. & 6 & 5 & 36.1 & 14.5 \\
\hline D. anthonyi & 2 & 6 & 12.0 & 17.4 \\
\hline D. nigrilore & 1 & 13 & 6.0 & 37.8 \\
\hline Prionochilus olivaceus & 3 & 0 & 18.1 & 0.0 \\
\hline \multicolumn{5}{|l|}{ Family Zosteropidae } \\
\hline Hypocryptadius cinnamomeus & 3 & 20 & 18.1 & 58.1 \\
\hline Lophozosterops goodfellowi & 0 & 11 & 0.0 & 32.0 \\
\hline Zosterops montamus & 1 & & 6.0 & 14.5 \\
\hline Net-days & 166.25 & 344 & - & - \\
\hline Total species & 30 & 35 & - & - \\
\hline Total individuals & 129 & 240 & 775.9 & 697.7 \\
\hline
\end{tabular}

evolutionary processes affecting communities (Brown, 1995; Heaney, 2001). Patterns of species replacements provide insights into competition from limitation by habitat preference or physical factors (Terborgh, 1971). More generally, information spread across altitudinal gradients provides a series of natural "experiments" in which species combinations are placed in different environmental contexts, allowing testing of ideas about causal factors. For these reasons, we next discuss generalities of patterns observed in the Kitanglad avifauna.

Adequacy of Sampling along the Elevational Transect-The degree to which our inventories summarize accurately the entire avifauna varies. The disturbed lowland habitats were least well sampled because of limited time and logistic problems. The 2 highest points (1900- and 2250$\mathrm{m}$ ridges) were probably sampled very thoroughly because their avifaunas are relatively simple and sampling was intensive, although detailed daily lists necessary for quantitative assessment were not made. For the 1100-, 1700-, and 1800-m ridges, however, we were able to evaluate the completeness of each single-elevation inventory (Fig. 3) using probabilistic estimators of total fauna size (Peterson \& Slade, 1998). In each of the inventories, the cumulative species total was approaching an asymptote (Fig. 3), suggesting that inventories were almost complete. Estimated percent completeness for the 3 ridges was $95.2 \%$ for the $1100-\mathrm{m}$ ridge, $94.7 \%$ for the $1700-\mathrm{m}$ ridge, and $91.5 \%$ for the $1800-\mathrm{m}$ ridge. Therefore, at least for the middle and upper portions of the transect, the Field Museum inventories appear to represent a fairly complete picture of the avifauna present on that slope of Kitanglad in the season in which our inventories were carried out.
Avifaunal Turnover along the Transect-Relatively little altitudinal turnover was observed along the $1550-\mathrm{m}$ altitudinal transect that we studied, coincident with patterns observed in mammal faunas along the same transect (Heaney, 2001). Migrant species were concentrated generally in the lowlands. A few species were restricted to the higher portions of the transect, such as Basilornis miranda, Centropus melanops, and Gallus gallus (above $1300 \mathrm{~m}$ ). On the mountain's northeastern flank, the steepness of the river valleys below $1000 \mathrm{~m}$ places highland and lowland habitats in close proximity, apparently explaining why some montane bird species (e.g., Leonardina woodi, Ptilocichla mindanensis, Rhinomyias goodfellowi, Aethopyga boltoni) can occasionally be observed at lower elevations along these valleys. Relatively few species (e.g., Batrachostomus septimus) appeared to be restricted to exclusively lowland forest habitats, probably a consequence of the already-great reduction of lowland forests in the region, given that numerous open-country species were restricted to this lower portion of the transect.

Elevational Overlap of Congeners - The FMNH surveys at 1100-, 1200-, 1300-, 1500-, 1800-, 1850-, 1900-, and 2250-m elevations provide a comparable set of inventories across an elevational range from near the lower limit of forest in the area up to near tree line (Table 6). Six pairs (Accipiter, Aplonis, Ficedula, Otus, Rhabdomis, Rhipidura), 2 trios (Aethopyga, Phapitreron), and 1 quintet (Dicaeum) of congeneric species were present along the transect. Overlapping congener distributions were the rule-the most extreme example was the genus Dicaeum, in which 5 species overlapped broadly at the $1100-1500-\mathrm{m}$ 
T.BIL 5. Summary of species observed participating in multispecies flocks at 2 different elevational levels on Mt. Kitanglad by the 1992 FMNH leam [ATP. RF]. Number of nocks $=15(11001300 \mathrm{~m})$ and $10(1500-1800 \mathrm{~m})$.

\begin{tabular}{|c|c|c|c|c|}
\hline \multirow[b]{2}{*}{ Species } & \multicolumn{2}{|c|}{ Number of individuals } & \multicolumn{2}{|c|}{ Percent of flocks } \\
\hline & $1100-1300 \mathrm{~m}$ & $1500-1800 \mathrm{~m}$ & $1100-1300 \mathrm{~m}$ & $1500-1800 \mathrm{~m}$ \\
\hline \multicolumn{5}{|l|}{ Family Columbidae } \\
\hline Phapitreron spp. & 5 & 0 & 33.3 & 0 \\
\hline Prilinopus occipitalis & 3 & 0 & 20.0 & 0 \\
\hline \multicolumn{5}{|l|}{ Family Psittacidae } \\
\hline $\begin{array}{l}\text { Prioniturus montamus } \\
\text { Family Apodidae }\end{array}$ & 0 & 2 & 0 & 20 \\
\hline $\begin{array}{l}\text { Family Apodidae } \\
\text { Collocalia esculenta }\end{array}$ & 0 & 1 & 0 & 10 \\
\hline \multicolumn{5}{|l|}{ Family Picidae } \\
\hline Dendrocopus maculatus & 5 & 1 & 33.3 & 10 \\
\hline Dryocopus javensis & 2 & 0 & 13.3 & 0 \\
\hline \multicolumn{5}{|l|}{ Family Campephagidae } \\
\hline Coracina megregori & 9 & 5 & 60.0 & 50 \\
\hline Pericrocrotus flammeus & 1 & 0 & 6.7 & 0 \\
\hline \multicolumn{5}{|l|}{ Family Pycnonotidae } \\
\hline $\begin{array}{l}\text { Hypsipetes philippinus } \\
\text { Family Dicuridae }\end{array}$ & 4 & 0 & 26.7 & 0 \\
\hline Dicrurus hottentottus & 2 & 0 & 13.3 & 0 \\
\hline \multicolumn{5}{|l|}{ Family Paridae } \\
\hline \multirow{2}{*}{\multicolumn{4}{|c|}{ Family Sittidae }} & 60 \\
\hline $\begin{array}{l}\text { Family Sittidae } \\
\text { Sitta oenochlamys }\end{array}$ & & & & 50 \\
\hline \multicolumn{5}{|l|}{ Family Rhapdornithidae } \\
\hline Rhabdomis inomatus & 3 & 0 & 20.0 & 0 \\
\hline R. mystacalis & 1 & 0 & 6.7 & 0 \\
\hline \multicolumn{5}{|l|}{ Family Sylvidae } \\
\hline Orthotomus heterolaemus & 0 & 1 & 0 & 10 \\
\hline Phylloscopus olivaceus & 3 & 3 & 20.0 & 30 \\
\hline \multicolumn{5}{|l|}{ Family Muscicapidae } \\
\hline Eumyias panayensis & 1 & 1 & 6.7 & 10 \\
\hline Ficedula hyperythra & 1 & 0 & 6.7 & 0 \\
\hline F. westermanni & 0 & 1 & 0 & 10 \\
\hline Rhinomyias goodfellowi & 1 & 0 & 6.7 & 0 \\
\hline Rhipidura nigrocinnamomea & 14 & 5 & 93.3 & 50 \\
\hline \multicolumn{5}{|l|}{ Family Pachycephalidae } \\
\hline Pachycephala philippinus & 1 & 2 & 6.7 & 20 \\
\hline \multicolumn{5}{|l|}{ Family Sturnidae } \\
\hline Aplonis panayensis & 2 & 0 & 13.3 & 0 \\
\hline Basilomis miranda & 1 & 1 & 6.7 & 10 \\
\hline \multicolumn{5}{|l|}{ Family Nectarinidae } \\
\hline Aethopyga boltoni & 0 & 1 & 0 & 10 \\
\hline A. primigenius & 11 & 3 & 73.3 & 30 \\
\hline Arachnothera clarae & 2 & 0 & 13.3 & 0 \\
\hline \multicolumn{5}{|l|}{ Family Dicaedae } \\
\hline Dicaeum spp. & 8 & 0 & 53.3 & 0 \\
\hline D. anthonyi & 1 & 0 & 6.7 & 0 \\
\hline D. nigrilore & 0 & 2 & 0 & 20 \\
\hline \multicolumn{5}{|l|}{ Family Zosteropidae } \\
\hline Hypocryptadius cinnamomeus & 7 & 3 & 46.7 & 30 \\
\hline Lophozosterops goodfellowi & 0 & 2 & 0 & 20 \\
\hline Zosterops montanus & 7 & 5 & 46.7 & 50 \\
\hline \multicolumn{5}{|l|}{ Family Fringillidae } \\
\hline Pyrrhula leucogastra & 0 & 1 & 0 & 10 \\
\hline
\end{tabular}

a This rather odd record is backed up by independent observations by DHC, again of an individual swiftlet foraging in concert with a multispecies flock. 

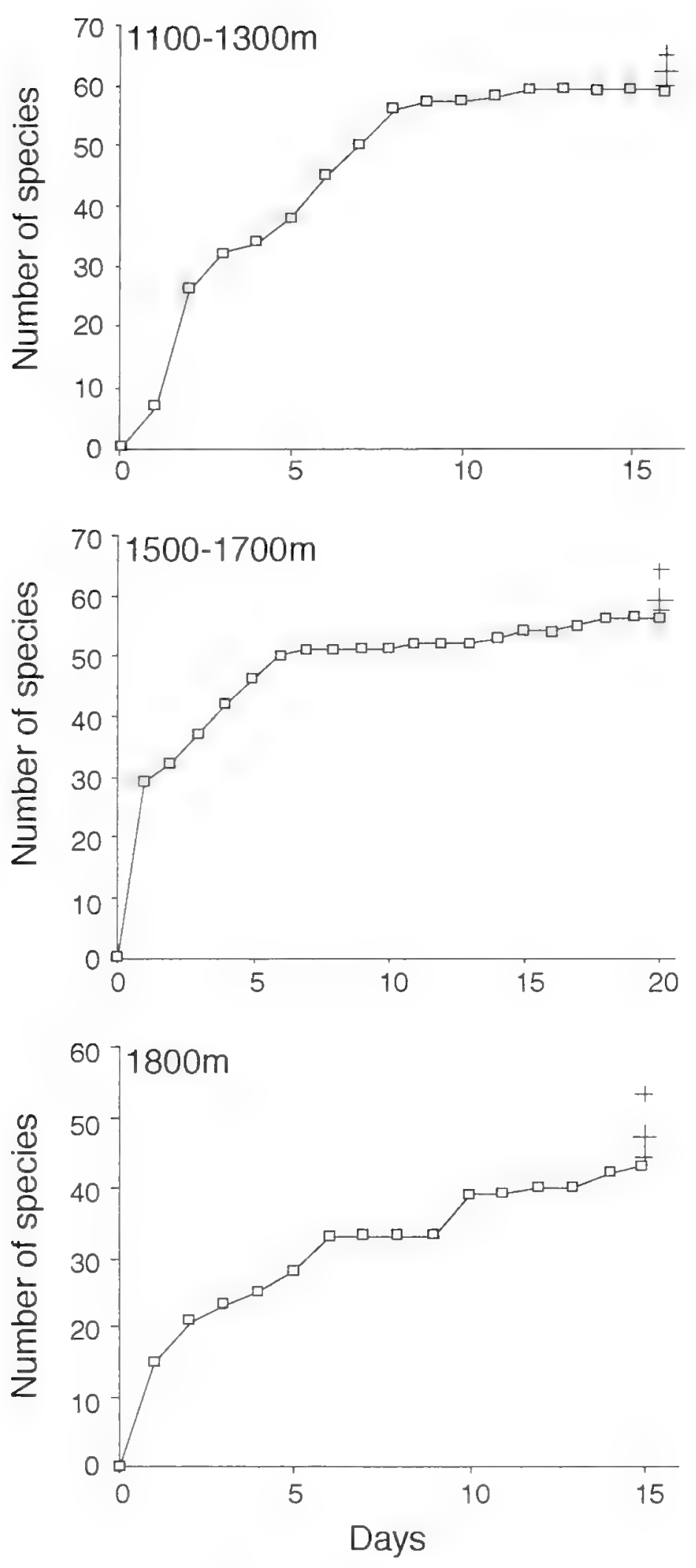

FIG. 3. Species-accumulation curves for 3 sites of detailed study: the 1100-1300-, 1500-1700-, and 1800$m$ ridges studied by the Field Museum team in 1992. Cumulative numbers of species recorded are shown as squares; $25 \%$ and $75 \%$ intervals (small $+s$ ) and the median (large $+s$ ) of the bootstrapped predicted overall species diversity are shown for the last day of the inventories.

stations on the transect. More generally, congeneric species pairs overlapped by 1-6 stations in all cases.

Over a broader spectrum of habitats, including the highly disturbed lowlands, numerous examples of nonoverlapping distributions were present. Each, however, consisted of related species with markedly different habitat requirements. For example, Lanius cristatus and L. schach were restricted to open, disturbed areas in the lowlands, whereas $L$. validirostris was found only in closed montane forest above $1800 \mathrm{~m}$, separated by about $1000 \mathrm{~m}$ of elevation from the other 2 species. Similarly, Centropus viridis was found in disturbed lowland forest below $1000 \mathrm{~m}$, whereas C. melanops was restricted to closed montane forest above $1500 \mathrm{~m}$ (which is itself a most surprising result). Other examples include members of the genera Orthotomus, Coracina, and Zosterops. Hence, elevational structuring of avifaunas in the absence of discrete habitat differences, which may be a strong indicator of competitive interactions (Terborgh, 1971), was not found in any group in the Kitanglad avifauna.

Altitudinal Patterns of Diversity and Endemism-Previous studies have documented a general pattern of high species richness in the lowlands, contrasting with elevated endemism in the highlands (e.g., Mayr, 1963; Peterson et al., 1993). Based on general references available (Dickinson et al., 1991), we estimate that approximately 172 of the species detected are resident and breeding on Kitanglad. Altitudinal patterns of diversity and endemism along the FMNH transect up Mt. Kitanglad (500-2250 m), however, do not show clearly the predicted inverse correlation of diversity and endemism (Fig. 4). Species richness was low at the lowest elevations studied $(\leq 700 \mathrm{~m})$, high in lowland and foothill forests $(1100-1300 \mathrm{~m})$ and at middle elevations $(1700-1800 \mathrm{~m})$, and declined somewhat at high elevations (1850-2250 m). We attribute the low species richness in the lowlands to the severe disturbance of natural habitats below $700-1000 \mathrm{~m}$ in the region, reducing the richness of those communities considerably, an explanation supported by the nondetection of numerous lowland species in recent surveys (e.g., Eurystomus orientalis), although many possible explanations exist (Heaney, 2001).

Philippine endemism was more than $35 \%$ in the lowlands (although our "lowland" surveys surely were affected by the near-complete deforestation at low elevations), rising to $100 \%$ at $1900 \mathrm{~m}$. Endemism at the level of Greater Mindanao and at the level of Mindanao alone was quite low in the lowlands but rose steadily to $40-50 \%$ at the highest elevations. Hence, ende- 
TABLE 6. Summary of species recorded at 8 elevational stations along the transect covered by the 1992-1993 FMNH groups.

\begin{tabular}{|c|c|c|c|c|c|c|c|c|}
\hline Species & $\begin{array}{c}1100-m \\
\text { camp }\end{array}$ & $\begin{array}{c}1200-m \\
\text { ridge }\end{array}$ & $\begin{array}{c}1300-m \\
\text { ridge }\end{array}$ & $\begin{array}{l}1500-m \\
\text { ridge }\end{array}$ & $\begin{array}{c}1800-\mathrm{m} \\
\text { ridge }\end{array}$ & $\begin{array}{c}1850-m \\
\text { ridge }\end{array}$ & $\begin{array}{c}1900-m \\
\text { camp }\end{array}$ & $\begin{array}{c}2250-m \\
\text { camp }\end{array}$ \\
\hline \multicolumn{9}{|l|}{ Family Accipitridae } \\
\hline Accipiter trivirgatus & $\mathrm{X}$ & - & - & $\mathrm{X}$ & - & - & - & - \\
\hline A. virgatus & $\mathrm{X}$ & $\mathrm{X}$ & & $\mathrm{X}$ & $\mathrm{X}$ & - & -- & - \\
\hline Haliastur indus & - & - & $\mathrm{X}$ & - & & & - & - \\
\hline Hieraaetus kienerii & $\mathrm{X}$ & $\mathrm{X}$ & - & - & - & - & - & - \\
\hline Spilornis holospilus & $\mathrm{X}$ & $\mathrm{X}$ & $\mathrm{X}$ & $\mathrm{X}$ & $\mathrm{X}$ & - & $\mathrm{X}$ & $\mathrm{X}$ \\
\hline \multicolumn{9}{|l|}{ Family Falconidae } \\
\hline Microhierax erythrogenys & - & - & - & $\mathrm{X}$ & & - & - & - \\
\hline \multicolumn{9}{|l|}{ Family Phasianidae } \\
\hline Gallus gallus & - & & $X$ & $\mathrm{X}$ & & - & $\mathrm{X}$ & $\mathrm{X}$ \\
\hline \multicolumn{9}{|l|}{ Family Columbidae } \\
\hline Chalcophaps indica & $\mathrm{X}$ & - & - & $\mathrm{X}$ & - & - & - & - \\
\hline Ducula poliocephala & $\mathrm{X}$ & - & - & $\mathrm{X}$ & $\mathrm{X}$ & - & - & - \\
\hline Macropygia temuirostris & $\mathrm{X}$ & - & $\mathrm{X}$ & $\mathrm{X}$ & $\mathrm{X}$ & - & $\mathrm{X}$ & $\mathrm{X}$ \\
\hline Phapitreron spp. & $\mathrm{X}$ & - & - & $\mathrm{X}$ & - & - & - & - \\
\hline P. amethystina & $\mathrm{X}$ & - & - & $\mathrm{X}$ & $\mathrm{X}$ & - & - & - \\
\hline Ptilinopus occipitalis & $\mathrm{X}$ & $\mathrm{X}$ & $\mathrm{X}$ & $\mathrm{X}$ & $\mathrm{X}$ & $\mathrm{X}$ & $\mathrm{X}$ & - \\
\hline \multicolumn{9}{|l|}{ Family Psittacidae } \\
\hline Loriculus philippensis & $\mathrm{X}$ & $\mathrm{X}$ & $\mathrm{X}$ & - & - & - & - & - \\
\hline Prioniturus waterstradti & $\mathrm{X}$ & $\mathrm{X}$ & $\mathrm{X}$ & $\mathrm{X}$ & $\mathrm{X}$ & - & - & $\mathrm{X}$ \\
\hline \multicolumn{9}{|l|}{ Family Cuculidae } \\
\hline Cacomantis sepulcralis & $\mathrm{X}$ & $\mathrm{X}$ & - & $\mathrm{X}$ & $X$ & $X$ & $\mathrm{X}$ & $\mathrm{X}$ \\
\hline Centropus melanops & - & & - & $\mathrm{X}$ & $\mathrm{X}$ & $\mathrm{X}$ & - & - \\
\hline Cuculus fugax & - & - & - & $\mathrm{X}$ & $\mathrm{X}$ & - & - & - \\
\hline \multicolumn{9}{|l|}{ Family Strigidae } \\
\hline Otus megalotis & $\mathrm{X}$ & $\mathrm{X}$ & $\mathrm{X}$ & $\mathrm{X}$ & - & - & 一 & - \\
\hline O. mirus & $\mathrm{X}$ & $\mathrm{X}$ & - & $\mathrm{X}$ & - & - & $\mathrm{X}$ & $\mathrm{X}$ \\
\hline \multicolumn{9}{|l|}{ Family Podargidae } \\
\hline Batrachostomus septimus & $\mathrm{X}$ & - & - & - & - & - & - & - \\
\hline \multicolumn{9}{|l|}{ Family Caprimulgidae } \\
\hline Caprimulgus manillensis & - & - & - & $\mathrm{X}$ & $\mathrm{X}$ & - & - & - \\
\hline \multicolumn{9}{|l|}{ Family Apodidae } \\
\hline Collocalia esculenta & $\mathrm{X}$ & $\mathrm{X}$ & $\mathrm{X}$ & $\mathrm{X}$ & $\mathrm{X}$ & $\mathrm{X}$ & $\mathrm{X}$ & $\mathrm{X}$ \\
\hline Hirundapus giganteus & - & - & - & $\mathrm{X}$ & -. & - & - & - \\
\hline \multicolumn{9}{|l|}{ Family Trogonidae } \\
\hline Harpactes ardens & $\mathrm{X}$ & - & $\mathrm{X}$ & $\mathrm{X}$ & $\mathrm{X}$ & $\mathrm{X}$ & $\mathrm{X}$ & - \\
\hline \multicolumn{9}{|l|}{ Family Bucerotidae } \\
\hline Aceros leucocephalus & $\mathrm{X}$ & $\mathrm{X}$ & - & - & - & - & - & - \\
\hline Buceros hydrocorax & $\mathrm{X}$ & $\mathrm{X}$ & $\mathrm{X}$ & $\mathrm{X}$ & $\mathrm{X}$ & $\mathrm{X}$ & $\mathrm{X}$ & $\mathrm{X}$ \\
\hline Penelopides panini & $\mathrm{X}$ & - & -- & - & - & - & $\mathrm{X}$ & - \\
\hline \multicolumn{9}{|l|}{ Family Picidae } \\
\hline Chrysocolaptes lucidus & $\mathrm{X}$ & $\mathrm{X}$ & - & $\mathrm{X}$ & $\mathrm{X}$ & - & - & - \\
\hline Dendrocopos maculatus & $\mathrm{X}$ & $\mathrm{X}$ & $\mathrm{X}$ & $\mathrm{X}$ & $\mathrm{X}$ & - & - & - \\
\hline Dryocopus javensis & $\mathrm{X}$ & - & - & $\mathrm{X}$ & - & - & $\mathrm{X}$ & - \\
\hline \multicolumn{9}{|l|}{ Family Campephagidae } \\
\hline Coracina megregori & $\mathrm{X}$ & $\mathrm{X}$ & $\mathrm{X}$ & $\mathrm{X}$ & $\mathrm{X}$ & $\mathrm{X}$ & $\mathrm{X}$ & - \\
\hline Lalage nigra & $\mathrm{X}$ & - & - & $\mathrm{X}$ & & - & - & - \\
\hline Pericrocotus flammeus & $\mathrm{X}$ & - & - & - & & - & -- & - \\
\hline \multicolumn{9}{|l|}{ Family Pycnonotidae } \\
\hline Hypsipetes philippinus & $\mathrm{X}$ & $\mathrm{X}$ & $X$ & $\mathrm{X}$ & $\mathrm{X}$ & $\mathrm{X}$ & $\mathrm{X}$ & - \\
\hline Family Dicuridae & & & & & & & & \\
\hline Dicrurus hottentottus & $\mathrm{X}$ & - & - & - & - & - & $X$ & - \\
\hline Family Oriolidae & & & & & & & & \\
\hline Irena cyanogaster & $\mathrm{X}$ & $\mathrm{X}$ & - & - & - & & - & - \\
\hline Family Paridae & & & & & & & & \\
\hline Parus elegans & $\mathrm{X}$ & $\mathrm{X}$ & $\mathrm{X}$ & $\mathrm{X}$ & $\mathrm{X}$ & $X$ & $X$ & $\mathrm{X}$ \\
\hline Family Sittidae & & & & & & & & \\
\hline $\begin{array}{l}\text { Sitta oenochlamys } \\
\text { Family Rhabdornithidae }\end{array}$ & $\mathrm{X}$ & $\mathrm{X}$ & $\mathrm{X}$ & $\mathrm{X}$ & $\mathrm{X}$ & - & $\mathrm{X}$ & $\mathrm{X}$ \\
\hline
\end{tabular}




\begin{tabular}{|c|c|c|c|c|c|c|c|c|}
\hline Species & $\begin{array}{l}1100-m \\
\text { camp }\end{array}$ & $\begin{array}{c}1200-\mathrm{m} \\
\text { ridge }\end{array}$ & $\begin{array}{c}1300-m \\
\text { ridge }\end{array}$ & $\begin{array}{c}1500-m \\
\text { ridge }\end{array}$ & $\begin{array}{l}1800-m \\
\text { ridge }\end{array}$ & $\begin{array}{l}1850-m \\
\text { ridge }\end{array}$ & $\begin{array}{l}\text { 1900-m } \\
\text { camp }\end{array}$ & $\begin{array}{c}2250-m \\
\text { camp }\end{array}$ \\
\hline Rhabdornis inornatus & $\mathrm{X}$ & $\mathrm{x}$ & & - & & & & \\
\hline R. mystacalis & $\mathrm{X}$ & - & - & - & - & - & $\mathrm{X}$ & \\
\hline \multicolumn{9}{|l|}{ Family Timaliidae } \\
\hline Leonardina woodi & $\mathrm{X}$ & $\mathrm{X}$ & - & $\mathrm{X}$ & - & - & $\mathrm{X}$ & $\mathrm{X}$ \\
\hline Macronous striaticeps & $\mathrm{X}$ & $\mathrm{X}$ & - & $\mathrm{X}$ & $\mathrm{X}$ & - & - & - \\
\hline \multicolumn{9}{|l|}{ Family Turdidae } \\
\hline Brachypteryx montana & - & & - & $\mathrm{X}$ & $\mathrm{X}$ & $\mathrm{X}$ & $\mathrm{X}$ & $\mathrm{X}$ \\
\hline Turdus poliocephalus & - & - & - & $X$ & $\mathrm{X}$ & $\mathrm{X}$ & $\mathrm{X}$ & $\mathrm{X}$ \\
\hline \multirow{2}{*}{\multicolumn{9}{|c|}{ Family Sylvidae }} \\
\hline Bradypterus caudatus & & & - & $\mathrm{X}$ & $\mathrm{X}$ & & & - \\
\hline Orthotomus heterolaemus & - & - & - & $\mathrm{X}$ & $\mathrm{X}$ & $\mathrm{X}$ & $\mathrm{X}$ & $\mathrm{X}$ \\
\hline Phylloscopus olivaceus & $\mathrm{X}$ & $\mathrm{X}$ & $\mathrm{X}$ & $\mathrm{X}$ & $\mathrm{X}$ & $\mathrm{X}$ & $\mathrm{X}$ & $\mathrm{X}$ \\
\hline \multicolumn{9}{|l|}{ Family Muscicapidae } \\
\hline Culicicapa helianthea & - & - & - & $\mathrm{X}$ & - & - & - & - \\
\hline Eumyias panayensis & $\mathrm{X}$ & $\mathrm{X}$ & $\mathrm{X}$ & $\mathrm{X}$ & $\mathrm{X}$ & - & $\mathrm{X}$ & $\mathrm{X}$ \\
\hline Ficedula hyperythra & $\mathrm{X}$ & $\mathrm{X}$ & $\mathrm{X}$ & $\mathrm{X}$ & $\mathrm{X}$ & $\mathrm{X}$ & $\mathrm{X}$ & $\mathrm{X}$ \\
\hline F. westermanni & $\mathrm{X}$ & $\mathrm{X}$ & - & $\mathrm{X}$ & $\mathrm{X}$ & $\mathrm{X}$ & - & $\mathrm{X}$ \\
\hline Muscicapa griseisticta & - & - & - & $\mathrm{X}$ & - & - & - & - \\
\hline Rhinomyias goodfellowi & $\mathrm{X}$ & $\mathrm{X}$ & - & $\mathrm{X}$ & $\mathrm{X}$ & - & - & - \\
\hline Rhipidura nigrocinnamomea & $\mathrm{X}$ & $\mathrm{X}$ & $\mathrm{X}$ & $\mathrm{X}$ & $\mathrm{X}$ & $\mathrm{X}$ & $\mathrm{X}$ & $\mathrm{X}$ \\
\hline R. superciliaris & $\mathrm{X}$ & $\mathrm{X}$ & $\mathrm{X}$ & $\mathrm{X}$ & $\mathrm{X}$ & $\mathrm{X}$ & - & \\
\hline \multicolumn{9}{|l|}{ Family Pachycephalidae } \\
\hline Pachycephala philippinensis & $\mathrm{X}$ & $\mathrm{X}$ & $\mathrm{X}$ & $\mathrm{X}$ & $\mathrm{X}$ & $\mathrm{X}$ & $\mathrm{X}$ & $\mathrm{X}$ \\
\hline \multicolumn{9}{|l|}{ Family Laniidae } \\
\hline Lanius validirostris & - & - & - & - & $\mathrm{X}$ & $\mathrm{X}$ & $\mathrm{X}$ & $\mathrm{X}$ \\
\hline \multicolumn{9}{|l|}{ Family Sturnidae } \\
\hline Aplonis minor & $\mathrm{X}$ & $\mathrm{X}$ & $\mathrm{X}$ & $\mathrm{X}$ & $\mathrm{X}$ & 一 & $\mathrm{X}$ & - \\
\hline A. panayensis & $\mathrm{X}$ & $\mathrm{X}$ & & & & & - & \\
\hline Basilomis miranda & - & - & $\mathrm{X}$ & $\mathrm{X}$ & $\mathrm{X}$ & $\mathrm{X}$ & $\mathrm{X}$ & $\mathrm{X}$ \\
\hline \multicolumn{9}{|l|}{ Family Nectarinidae } \\
\hline Aethopyga boltoni & & - & - & - & $\mathrm{X}$ & $\mathrm{X}$ & $\mathrm{X}$ & $\mathrm{X}$ \\
\hline A. primigenia & $\mathrm{X}$ & $\mathrm{X}$ & $\mathrm{X}$ & $\mathrm{X}$ & $\mathrm{X}$ & $\mathrm{X}$ & $\mathrm{X}$ & - \\
\hline A. shelleyi & — & - & - & & $\mathrm{X}$ & - & - & \\
\hline Arachnothera clarae & $\mathrm{X}$ & $\mathrm{X}$ & - & - & - & - & $\mathrm{X}$ & - \\
\hline \multicolumn{9}{|l|}{ Family Dicaedae } \\
\hline Dicaeum anthonyi & $\mathrm{X}$ & $\mathrm{X}$ & - & $\mathrm{X}$ & - & - & $\mathrm{X}$ & \\
\hline D. bicolor & $\mathrm{X}$ & $\mathrm{X}$ & - & $\mathrm{X}$ & - & - & - & - \\
\hline D. hypoleucum & $\mathrm{X}$ & $\mathrm{X}$ & - & $\mathrm{X}$ & - & 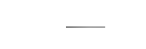 & - & \\
\hline D. nigrilore & $\mathrm{X}$ & $\mathrm{X}$ & - & $\mathrm{X}$ & $\mathrm{X}$ & $\mathrm{X}$ & $\mathrm{X}$ & $\mathrm{X}$ \\
\hline D. trigonostigma & $\mathrm{X}$ & & - & $\mathrm{X}$ & - & - & - & \\
\hline Prionochilus olivaceus & $\mathrm{X}$ & & & & - & - & & \\
\hline \multicolumn{9}{|l|}{ Family Zosteropidae } \\
\hline Hypocryptadius cinnamomeus & $\mathrm{X}$ & $\mathrm{X}$ & $\mathrm{X}$ & $\mathrm{X}$ & $\mathrm{X}$ & $\mathrm{X}$ & $\mathrm{X}$ & $\mathrm{X}$ \\
\hline Lophozosterops goodfellowi & & - & - & $\mathrm{X}$ & $\mathrm{X}$ & $\mathrm{X}$ & $\mathrm{X}$ & $\mathrm{X}$ \\
\hline Zosterops montanus & $X$ & $\mathrm{X}$ & $\mathrm{X}$ & $X$ & $\mathrm{X}$ & $\mathrm{X}$ & $\mathrm{X}$ & $\mathrm{X}$ \\
\hline \multicolumn{9}{|l|}{ Family Fringillidae } \\
\hline Pyrrhula leucogenys & - & - & - & $\mathrm{X}$ & $\mathrm{X}$ & $\mathrm{X}$ & $\mathrm{X}$ & $\mathrm{X}$ \\
\hline Total & 56 & 40 & 27 & 58 & 44 & 27 & 39 & 28 \\
\hline
\end{tabular}

mism, as expected on the basis of other transects, increased steadily with elevation.

Single-Site InVEntory - Intensive single-site inventories provide extremely valuable data for conservation planning (Balmford \& Gaston, 1999). Thus, the total of 198 species now known to occur (or to have occurred) on the Kitanglad massif ranks it among the richest sites for birds in the Philippines, surpassing such well-surveyed sites on
Luzon as Mt. Isarog, with 135 species (Goodman \& Gonzales, 1989). While such statements demand detailed comparisons of survey effort, altitudinal range surveyed, habitat range surveyed, and so on, the crude comparison remains - the Kitanglad massif holds a large number of species in comparison with other well-surveyed sites.

In fact, given that the Kitanglad massif is much smaller in area than the Sierra Madre of 


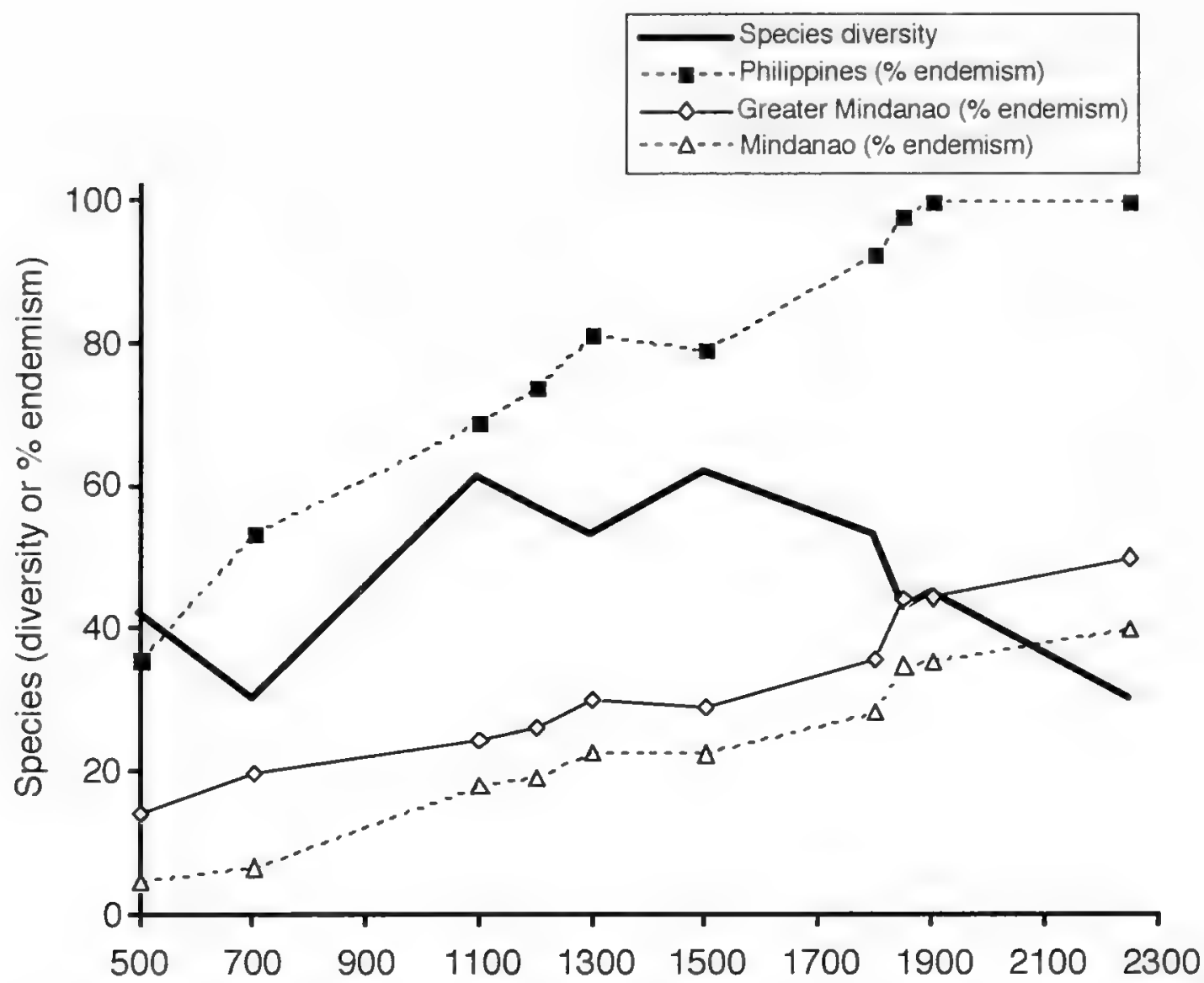

FlG. 4. Elevational biodiversity trends: species diversity, Philippine endemism, Greater Mindanao endemism, and strict Mindanao endemism, all plotted against elevation (in $\mathrm{m}$ ) along the 500-2250-m transect covered by the FMNH expeditions.

Luzon, Kitanglad's species richness may be comparable with the total of $\sim 250$ species believed to occur there (Poulsen, 1995). Compared with these sites, Kitanglad's avifauna is similar in richness at lower elevations but richer toward higher elevations. Given the relatively broad elevational distributions of Philippine birds, we suspect that approximately 200 species may represent a limit for single lowland-to-highland transect avian diversity in the Philippines.

Avifaunistic Changes - Of the $\sim 172$ bird species known to be resident in the Kitanglad region, the early collectors (most prominently Salomonsen, Rabor, and Gonzales) collected 129 (75.0\%) (and probably sighted many more; overall field time not known). Recent field crews, during almost 4 months of intensive fieldwork at all elevations holding significant forest, as well as many months of effort by bird-watchers, detected $153(88.9 \%)$ species. Hence, the early surveys did not detect as high a proportion of overall known species richness as the modern ones. However, to understand how best to interpret the results of the early surveys, it is worth examining which groups of species were better detected by the early surveys and which were better detected by the modern work.
The 18 species documented historically but with no recent records in spite of intensive studies are Falco severus, Streptopelia bitorquata, Ninox philippensis, Hirundapus giganteus, Alcedo atthis, Halcyon capensis, Eurystomus orientalis, Eurylaimus steerii, Coracina morio, Lalage melanoleuca, Pycnonotus urostictus, Hypsipetes everetti, Oriolus steerii, Stachyris capitalis, Orthotomus nigriceps, Nectarinia sperata, Aethopyga pulcherrima, and Arachnothera longirostra (Table 2). Several additional species well documented by historical specimens are known from only 1 or 2 recent records (e.g., Ducula carola, Surniculus lugubris, Alcedo argentata, Zoothera andromedae). A substantial portion of these species (see Species Accounts) are lowland species (Dickinson et al., 1991) and may be in danger of extirpation from the Kitanglad region because of habitat destruction. Hence, the bulk of the species not detected in recent surveys appear to be species that would not be likely to be found in the reduced set of habitats - chiefly montane-remaining on the mountain today (an exception would be $Z$. andromedae, which is an elusive montane forest resident).

Similar are a group of species that, while collected commonly in the 1960s (Ripley \& 
Rabor, 1961), have not been recorded in recent years on Kitanglad's accessible northeastern flank, above Dalwangan, despite the visits of many bird-watchers. These species appear restricted on Kitanglad to the relatively inaccessible northwestern and western flanks: all 3 Phapitreron, Columba vitiensis, Harpactes ardens, and Aceros lencocephalus. These species are all large-bodied frugivores, and it seems likely that their decline on Kitanglad is due to hunting pressure as well as to deforestation. It is noteworthy that no raptors feature in this group, suggesting that they are not heavily hunted, perhaps because of inconspicuousness or unpalatability.

Recent surveys, however, documented a total of 43 species not known historically. These species break down fairly clearly into 3 groups: water-associated species; species of open and disturbed habitats; and low-density, primary habitat species. Water-associated species were detected principally by $\mathrm{AG}$ and colleagues on the mountain's lower slopes, such as Butorides striatus, Dupetor flavicollis, Gallirallus philippensis, G. torquatus, Amaurornis olivacea, and $A$. phoenicurus. Most likely, the early surveys were carried out in areas lacking aquatic habitats, and for that reason these species were not collected.

Another component lacking from the early collections are resident and migrant species restricted to open and disturbed habitats, principally in the lowlands surrounding the Kitanglad massif. These species include, for example, Geopelia striata, Halcyon chloris, Merops philippinus, Anthreptes malacensis, and Zosterops everetti. These species were probably much less apparent around Kitanglad in the 1950s and 1960s, as habitat destruction was less far advanced than at present. In addition, they were probably of least interest to the collectors, being principally species that are common and widespread throughout the Philippines. Hence, the absence of these disturbed- and open-habitat species in the early collections may indicate the effects of colonization and advance by such species in the wake of widespread habitat destruction (Brooks et al., 1996).

Finally, we detected several low-density, primary forest bird species not noted in the early surveys, including Spizaetus cirrhatus, S. philippensis, Centropus melanops, Ducula poliocephala, Bolbopsittacus lumulatus, Pitta erythrogaster, Irena cyanogaster, and Rhipidura superciliaris. Especially surprising is the absence of early records of Buceros hydrocorax, which we found to be common in the forested portions of the massif and which would have been of particular interest to early collectors.

Hence, it appears that for the Mt. Kitanglad region, the early information available (all of it specimen based, Rabor's material being the principal part) does not present as exhaustive a sampling of the avifauna present as do modern surveys. This result apparently holds also for other groups that Rabor sampled, such as mammals, where the use of new methodologies has amply demonstrated that an enormous amount remains to be learned about sites earlier surveyed by Rabor (L. R. Heaney, pers. comm.). Nevertheless, the material accumulated by Rabor, Gonzales, Salomonsen, Bregulla, and other early collectors is remarkable in that most species were recorded as present; these records provide considerable confidence in the diversity of possible uses of their data for documenting bird distributions elsewhere in the Philippines. The data and specimens accumulated by Rabor and colleagues (with identifications appropriately checked and modernized) merit further exploration as to their utilityindeed, we observed no situations in which Rabor tag data were clearly suspect. We suggest that the data from all of these explorations would lay an unparalleled foundation for a national biological inventory of the Philippines.

Conservation of Mindanao's Endemic SpeciesEighteen bird species are strictly endemic to Mindanao. Seventeen of these endemics have been found on Kitanglad: Trichoglossus johnstoniae, Prioniturus waterstradti, Otus mirus, Actenoides hombroni, Coracina mcgregori, Leonardina woodi, Rhinomyias goodfellowi, Rhipidura nigrocinnamomea, Orthotomus heterolaemus, Basilornis miranda, Aethopyga primigenius, A. boltoni, Dicaeum nigrilore, D. propium, Lophozosterops goodfellowi, Hypocryptadius cinnamomeus, and Erythrura coloria. The only Mindanao endemic not found on Kitanglad, Aethopyga linarabori, was recently described as a species distinct from the more widespread Mindanao endemic $A$. boltoni (Kennedy et al., 1997) - it is restricted to the eastern provinces of Davao del Norte and Davao Oriental and thus is an allospecies of a Kitanglad resident species. As such, the Kitanglad massif is certainly comparable with Mt. Apo in terms of richness of Mindanao endemic species.

The flagship reserve in the southern Philippines has traditionally been Mt. Apo National 
Park, centered on a tall volcanic mountain $(2954 \mathrm{~m})$ at the southern extreme of the island. Mt. Apo was the first montane area of Mindanao to see extensive zoological exploration, and for that reason many species were long known only from there. Apo, however, is a slender volcanic cone and therefore has very limited area at higher elevations (approximately $388 \mathrm{~km}^{2}$ above $1000 \mathrm{~m}$ ). Those highest elevations of the mountain, moreover, have been compromised recently by the construction of a geothermal energy production plant, and the overall integrity of the region has been endangered by proximity to Davao City.

Kitanglad, in contrast with Apo, is a blockshaped massif (maximum elevation $2938 \mathrm{~m}$ ). It has extensive areas at high elevations (approximately $1757 \mathrm{~km}^{2}$ above $1000 \mathrm{~m}$ ), about 4.5 times as much as Apo, although, as on Apo, construction of installations around the peak (e.g., Bukidnon Telephone, Philippine Telephone and Telegraph, Philippine Army, Radio Mindanao Network, and the DLG Building) has jeopardized natural habitats at the highest elevations. With the documentation that the Kitanglad avifauna includes essentially the full complement of species unique to the island, we suggest that Kitanglad may prove to be a more appropriate primary focus for the conservation of Mindanao's montane species. Mt. Kitanglad National Park was declared by Proclamation No. 667 on 14 Dec. 1990 and is therefore under the management of the Philippine Department of the Environment and Natural Resources. It is also one of the Integrated Protected Areas Priority sites. The area proposed under the Global Environment Fund Conservation of Priority Protected Areas Project includes a buffer zone of 74,100 ha.

Specimens versus Observations - Avifaunal inventories typically fall clearly into 1 of 2 categories: those assembled by observation, at times aided by mist-net captures, versus those assembled via a combination of specimen collection (when possible) and observations. A third source of information is from historical specimens, although often neglected. Oddly enough, true bridges between these worlds in primary research studies, including both the force of numbers and time that observations by birdwatchers can offer and the force of information and depth that specimen-based ornithology provides, are few. One feature of the present monograph, then, is the presentation of such a bridge.
We have presented and discussed gaps in species lists assembled based on the historical specimen record and emphasized the need for new, modern survey initiatives. Use of mist-nets is key (see, e.g., the important nets-only records, such as Phapitreron brumeiceps), as is increased reliance on vocal cues for efficient species detection and identification (Parker, 1991). More generally, a focus on inventory (assembling a complete catalog of species present), rather than sampling or simply collecting specimens, is enormously helpful (Colwell \& Coddington, 1994; Peterson \& Slade, 1998). We have avoided a point-to-point comparison of which teams detected more species in this study, particularly given marked differences in time spent on the mountain, techniques employed, experience of observers, and elevations and slopes surveyed.

Bird-watchers clearly held a great advantage in terms of numbers of observers and time spent observing. Kitanglad is a relatively accessible mountain massif with abundant endemic species in a region that is itself rich in endemism and as such has attracted immense attention from the bird-watching community. The numerous teams of birders that have visited Kitanglad have reached several sectors of the range that would otherwise have gone unsurveyed and have provided broader seasonal coverage than the collecting teams were able to cover (although wet-season data for the mountain are still scanty; Fig. 2). In this sense, bird-watchers provided to this monograph an enormous force of observation effort that would otherwise not have been represented in this inventory. One shortcoming, though, is that exact locations, dates, and/or elevations were not always recorded.

Specimen collection and preparation is a timeintensive endeavor and clearly cannot in and of itself provide great numbers of records of species; for this reason, all the recent collecting teams that have visited Kitanglad have supplemented specimen records with observations. Observational information may have been collected by the earlier collecting teams, but this information was not available to us, if it exists. The specimen information summarized herein offers a powerful advantage in that records can be revisited in light of new information or new interpretations - as species limits are reinterpreted, for example, specimens can be determined precisely, whereas observational information cannot. Specimens provide a rich resource of additional information, including providing the basis for description and 
documentation of new taxa (e.g., Gamauf et al. 1998a; Kennedy et al., 1997, 2001), understanding species limits and phylogenetic relationships of complex groups (Miranda et al., 1997; Kemp, 1988), and understanding seasonal and reproductive status (Remsen, 1995).

\section{Conclusions}

The present study examined the avifauna of Mt. Kitanglad, a large mountain mass in northern Mindanao. The composition of $\mathrm{Mt}$. Kitanglad's avifauna has not previously been documented in detail. In this monograph, we present a summary of all information existing regarding the avifauna of the region; principal results and points for further consideration include the following:

1. Mt. Kitanglad holds one of the richest single-site avifaunas in the Philippines, with a total of 198 bird species, of which about 172 are resident. A further 10 species are considered hypothetical or doubtful as occurring on the mountain.

2. Of Kitanglad's avifauna, 85 species are endemic to the Philippines, 28 are endemic to Greater Mindanao, and 17 are endemic to Mindanao alone, all but one of the island's endemic species.

3. Of Kitanglad's avifauna, 1 species is considered critically endangered, 10 as vulnerable. 17 as near threatened, and 1 as data deficient (BirdLife International, 2004).

4. Changes in threat categorization (Collar et al., 1999) relative to those used in earlier publications (Collar et al., 1994) appear largely justified based on the evidence from Kitanglad.

5. Kitanglad's lower slopes have lost nearly all their forest species through deforestation and hunting, but the mountain's highelevation forests retain rich and intact communities of birds.

6. Great potential for further avian discoveries await on Mindanao's mountains, as illustrated by the newly described Scolopax bukidnonensis; odd and suggestive elevational records (e.g., Centropus melanops); first reports for the island of Luscinia calliope, Turdus chrysolaus, and Motacilla alba; and large range extensions for Phapitreron brumneiceps and Otus mirus.
7. Kitanglad appears to constitute a key site for conservation of montane birds on Mindanao, with excellent representation of the island's endemic species of birds, and as such its status as a protected area should be given full international support.

\section{Acknowledgments}

ATP, RF, JCTG, DHC, SEB, and NADM thank L. R. Heaney for making their studies on Kitanglad possible and for reading an early version of the manuscript. Thanks also to their numerous other field companions. We thank the 3 reviewers for their detailed comments and suggestions. Their fieldwork was funded by the MacArthur Foundation, the National Science Foundation, and the Marshall Field Fund, Field Museum of Natural History. AG thanks M. Preleuthner. A. Schuster. S. Tebbich, M. Zeiler. and $\mathrm{H}$. Winkler for field assistance: Mr. Enoch Arataya (San Antonio) and E. Makhanoy (Dalwangan) for their on-site support and C. Guyamara for his numerous comments regarding Philippine Eagles; and the PEFI (Philippine Eagle Foundation, Inc.) for accommodation at their lodgehouse (northeast slope). Her fieldwork (project on ecomorphology of Philippine birds of prey) was funded by the FWF Austria (BIO 8889). TB and GD thank C. Makhanoy for his assistance to their visit, which was a final part of the Cambridge Philippines Rainforest Project 1991: full acknowledgments for that work are given elsewhere (Evans et al., 1993).

The following people provided records, information or discussion that greatly improved the manuscript, and to whom we are extremely grateful: Des Allen, Naja Steen Andersen, Guy Anderson, Chris Bell, Finn Danielsen, Richard Fairbank, Matthias Fehlow, Tim Fisher, Ian Gardner, S. Hayhow, Mel Heath, Jon Hornbuckle, Jesper Hornskov, Franck Ishoi, Mikkel Kure Jakobsen, Bob Kennedy, Ben King, Jan Bolding Kristensen, Ian Lewis, Adrian Long, Andrew Pierce, Carsten Rahbek, Nigel Redman, Herbert Schifter, Paul Thompson, Rob Timmins, Michael Trasborg and Filip Verbelen. We also thank Nigel Collar, Mike Crosby, James Lowen, Joe Tobias, Eberhard Curio, Krys Kazmierczak, and Richard Thomas for providing literature and other information. Finally, we are extremely 
grateful to the curatorial staff of the museums providing information or access to collections (see Study Area and Methods).

\section{Literature Cited}

BAlmford, A., And K. J. Gaston. 1999. Why biodiversity surveys are good value. Nature, 398: 204-205.

BirdLIFE INTERNATIONAL 2004. Threatened Birds of the World 2. BirdLife International, Cambridge, U.K.

Brooks, T., AND G. DUtSon. 1997. Twenty-nine new island records of birds from the Philippines.

- Bulletin of the British Ornithologists' Club, 117: 32-37.

Brooks, T. M., G. C. L. Dutson, P. M. Magsalay, AND B. F. KING. 1996. An annotated checklist of the birds of Rajah Sikatuna National Park, Bohol. Forktail. 11: 121-134

Brooks, T. M., S. L. Pimm, and N. J. Collar. 1997. The extent of deforestation predicts the number of birds threatened with extinction in insular Southeast Asia. Conservation Biology, 11: 382-394.

Brown, J. H. 1995. Macroecology. University of Chicago Press, Chicago.

Carey, G., and U. Olsson. 1995. Field identification of Common, Wilson's, Pintail and Swinhoe's snipes. Birding World, 8: 179-190.

Chantler, P., and I. Driessens. 1994. Swifts of the World. Pica Press, Robertsbridge, U.K.

Clayton, D. H., and K. P. Johnson. 2000. Testing species limits of non-echolocating Philippine swiftlets (Collocalia spp.) using molecular genetic data. Sylvatrop Technical Journal of Philippine Ecosystems and Natural Resources, 10: 70-77.

Collar, N. J. 1997a. Species survival versus perpetuation of myth-The case of the Philippine Eagle. Newsletter of the World Working Group on Birds of Prey and Owls, 23/24: 23-26.

1997b. Species survival vs. perpetuation of myth-The case of the Philippine Eagle. Oryx, 31:4-7.

Collar, N. J., M. J. Crosby, and A. J. Stattersfield. 1994. Birds to Watch 2. The World List of Threatened Birds. BirdLife International, Cambridge, U.K.

Collar, N. J., N. A. D. Mallari, and B. R. Tabaranza. 1999. Threatened Birds of the Philippines: The Haribon Foundation/BirdLife International Red Data Book. Bookmark, Inc., Manila.

Colwell, R. K., And J. A. Coddington. 1994. Estimating terrestrial biodiversity through extrapolation. Philosophical Transactions of the Royal Society of London B, 335: 101-118.

Curio, E. 1997. Species Conservation as an Integral Part of Forest Maintenance in the Philippines: Third Report. Animal Behaviour Research Group, Rühr Universität, Bochum, Germany.

Dickerson, R. E., ED. 1928. Distribution of Life in the Philippines. Monograph 21. Bureau of Science, Manila.
Dickinson, E. C. 1989. A review of the larger Philippine swiftlets of the genus Collocalia. Forktail, 4: $19-53$.

Dickinson, E. C., R. S. Kennedy, and K. C. Parkes. 1991. The Birds of the Philippines. British Ornithologists' Union, Tring, U.K.

Dupont, J. E. 1971. Philippine Birds. Delaware Museum of Natural History, Greenville, Delaware.

Evans, T. D., G. C. L. Dutson, And T. M. Brooks. 1993. Final Report of the Cambridge Philippines Rainforest Project 1991. BirdLife International, Cambridge, U.K.

Gamauf, A., and M. Preleuthner. 1998. A new taxon of the Barred Honeybuzzard Pernis celebensis from the Philippines. Bulletin of the British Ornithologists' Club, 118: 90-101.

Gamauf, A., M. Preleuthner, and W. Pinsker. 1998a. Distribution and field identification of Philippine birds of prey: 1. Philippine Hawk Eagle Spizaetus philippensis and Changeable Hawk Eagle Spizaetus cirrhatus. Forktail, 14: 1-11.

Gamauf, A., M. Preleuthner, and H. Winkler. 1998b. Philippine birds of prey: Interrelations among habitat, morphology and behavior. Auk, 115: 713-726.

Gonzales, R. B. 1968. A study of the breeding biology and ecology of the monkey-eating eagle. Silliman Journal, 15: 461-500.

1971. Report on the 1969 status of the Monkey-eating Eagle on Mindanao Island, Philippines. Bulletin of the International Council for Bird Preservation, 11: 154-168.

Goodman, S. M., and P. C. Gonzales. 1989. The birds of Mt. Isarog National Park, southern Luzon, Philippines, with particular reference to altitudinal distribution. Fieldiana Zoology, 60: $1-39$.

Goodman, S. M., D. E. Willard, and P. C. Gonzales. 1995. The birds of Sibuyan Island, Romblon Province, Philippines, with particular reference to elevational distribution and biogeographic affinities. Fieldiana Zoology, 82: 1-57.

Gretton, A. 1990. Recent reports. Oriental Bird Club Bulletin, 11: 40-48.

HARrap, S., AND T. Fisher. 1994. A mystery woodcock from the Philippines. Oriental Bird Club Bulletin, 19: $54-56$.

HeAney, L. R. 1986. Biogeography of mammals in SE Asia: Estimates of rates of colonization, extinction and speciation. Biological Journal of the Linnean Society, 28: 127-165.

2001. Small mammal diversity along elevational gradients in the Philippines: An assessment of patterns and hypotheses. Global Ecology and Biogeography, 10: 15-39.

2002. A decade of research on Philippine mammals: Progress and challenges. Silliman Journal, 42: 88-108.

Heaney, L. R., and J. C. J. Regalado. 1998. Vanishing Treasures of the Philippine Rainforest. The Field Museum, Chicago. 
Heaney, L. R., E. K. Walker, B. R. Tabaranza, And N. Ingle. 2002. Mammalian diversity in the Philippines: An assessment of the adequacy of current data. Sylvatrop: Technical Journal of Philippine Ecosystems and Natural Resources, 10: 6-27.

HoRnSKOV, J. 1995. Recent observations of birds in the Philippine Archipelago. Forktail, 11: 1-10.

Juniper, T., And M. PARr. 1998. Parrots: A Guide to the Parrots of the World. Pica Press, Robertsbridge, U.K.

KEMP, A. C. 1988. The systematics and zoogeography of Oriental and Australasian hornbills (Aves: Bucerotidae). Bonner Zoologische Beiträge, 39: 315-345.

Kennedy, R. S., T. H. Fisher, S. C. B. Harrap, A. C. Diesmos, And A. S. Manamtam. 2001. A new species of woodcock (Aves: Scolopacidae) from the Philippines and a re-evaluation of Asian/Papuasian woodcock. Forktail, 17: 1-12.

Kennedy, R. S., P. C. Gonzales, E. C. Dickinson, H. C. J. Miranda, And T. H. Fisher. 2000. A Guide to the Birds of the Philippines. Oxford University Press, Oxford.

Kennedy, R. S., P. C. Gonzales, and H. C. J. Miranda. 1997. New Aethopyga sunbirds (Aves: Nectariniidae) from the island of Mindanao, Philippines. Auk, 114: 1-10.

Kennedy, R. S., And H. C. J. Miranda. 1998. In memoriam: Dioscoro S. Rabor, 1911-1996. Auk, 115: $204-205$.

KRUPA, R. E. 1985. Report on the birds of prey, pp. 263-276. In Proceedings of the Third East-Asian Bird Protection Conference. Wild Bird Society of Japan, Tokyo.

LAMBERT, F. R. 1993. Some key sites and significant records of birds in the Philippines and Sabah. Bird Conservation International, 3: 281-297.

1996. Pittas, Broadbills, and Asities. Pica Press, Sussex, U.K

MAYr, E. 1963. Animal Species and Evolution. Columbia University Press, New York.

Meyer de Schauensee, R. 1957. Notes on Philippine birds. Notulae Naturae, 303: 1-12.

Miranda, H. C, J., R. S. Kennedy, And D. P. Mindell. 1997. Phylogenetic placement of Mimizuku gurneyi (Aves: Strigidae) inferred from mitochondrial DNA. Auk, 114: 315-323.

Myers, N., R. A. Mittermeier, C. G. Mittermeier, G. A. B. DA Fonseca., And J. Kent. 2000. Biodiversity hotspots for conservation priorities. Nature, 403: 853-858.

Nordeco AND DENR, Integrating Conservation and Development in Protected Area Management. Mount Kitanglad Range National Park, the Philippines. NORDECO, Copenhagen.

PARKer, T. A., III. 1991. On the use of tape recorders in avifaunal surveys. Auk, 108: 443-444.

PARKeS, K. C. 1961. The Crested Lizard Hawk (Aviceda jerdoni) in the Philippines. Postilla, 51: 1-10.

1962. The Red Junglefowl of the PhilippinesNative or introduced? Auk, 79: 479-481.

Payne, R. B. 1997. Family Cuculidae (cuckoos), pp. 508-607. In del Hoyo, J., A. Elliott and J. Sargatal eds., Handbook of the Birds of the World, Vol. 4. Lynx Editions, Barcelona.
Peterson, A. T., And I. L. Brisbin. 1998. Genetic endangerment of wild Red Junglefowl Gallus gallus? Bird Conservation International, 8: 387-394.

Peterson, A. T., O. A. Flores V. L. S. Leon P., J. E. Llorente B., M. A. Luis M., A. G. NavarroSiguenza., M. G. Torres Ch., And I. Vargas F. 1993. Conservation priorities in northern Middle America: Moving up in the world. Biodiversity Letters, 1: 33-38.

Peterson, A. T., N. Ingle, and R. Fernandez. 1995. Notes on the nesting behavior of the White-bellied Woodpecker. Wilson Bulletin, 107: 182-184.

Peterson, A. T., And N. A. Slade. 1998. Extrapolating inventory results into biodiversity estimates and the importance of stopping rules. Diversity and Distributions, 4: 95-105.

Poulsen, M. K. 1995. The threatened and nearthreatened birds of Luzon, Philippines, and the role of the Sierra Madre mountains in their conservation. Bird Conservation International, 5: 79-115.

Preleuthner, M., and A. Gamauf. 1998. A possible new subspecies of the Philippine Hawk-Eagle (Spizaetus philippensis) and its future prospects. Journal of Raptor Research, 32: 126-135.

RABOR, D. S. 1965. The present status of the Monkeyeating Eagle Pithecophaga jefferyi Ogilvie-Grant of the Philippines, pp. 55-63. In IUCN, ed., Conference on Conservation of Nature and Natural Resources in Tropical South East Asia, Bangkok, Thailand, November 29-December 4, 1965. International Union for the Conservation of Nature and Natural Resources, Bangkok.

1968. The present status of the Monkey-eating Eagle Pithecophaga jefferyi Ogilvie-Grant of the Philippines. IUCN Publications, 10: 312-314.

1971. The present status of the Monkey-eating Eagle Pithecophaga jefferyi Ogilvie-Grant of the Philippines. Philippine Geographical Journal, 15: 90-103.

REMSEN, J. V. 1995. The importance of continued collecting of bird specimens to ornithology and bird conservation. Bird Conservation International, 5: $145-180$.

Remsen, J. V. JR., And D. A. Good. 1996. Misuse of data from mist-net captures to assess relative abundance of bird populations. Auk, 113: 381-398.

RIPLEY, S. D., AND D. S. RABOR. 1961. The avifauna of Mount Kitanglad. Postilla, 50: 1-20.

Robson, C. 1993. From the field. Oriental Bird Club Bulletin, 17: 49-53.

Salomonsen, F. 1953. Miscellaneous notes on Philippine birds. Videnskabelige meddelelser fra Dansk naturhistorisk forening i Kjøbenhavn, 115: 205-281.

Sibley, C. G., and B. L. J. Monroe. 1990. Distribution and Taxonomy of Birds of the World. Yale University Press, New Haven, Connecticut.

Soberón, J., And J. E. Llorente B. 1993. The use of species accumulation functions for the prediction of species richness. Conservation Biology, 7: 480-488.

Stattersfield, A. J., M. J. Crosby, A. J. Long, And D. C. WEGE. 1998. Endemic Bird Areas of the World: Priorities for Global Conservation. BirdLife International, Cambridge, U.K. 
TERBorgh, J. W. 1971. Distribution on environmental gradients: Theory and a preliminary interpretation of distributional patterns in the avifauna of the Cordillera Vilcabamba, Peru. Ecology, 52: 26-36.

U.S. Geological Survey. 2000. Global Land Cover 2000. U.S. Geological Survey, Washington, D.C.
Waldbauer, G. P., and S. M. Waldbauer. 1982. First sight record of the Spotted Dove Streptopelia chinensis from Luzon and a summary of its range expansion in the Philippines. Bulletin of the British Ornithologists' Club, 102: 22-24.

ZiMMER, J. T. 1918. A few rare birds from Luzon, Mindanao and Mindoro. Philippine Journal of Science, 13: 223-232. 


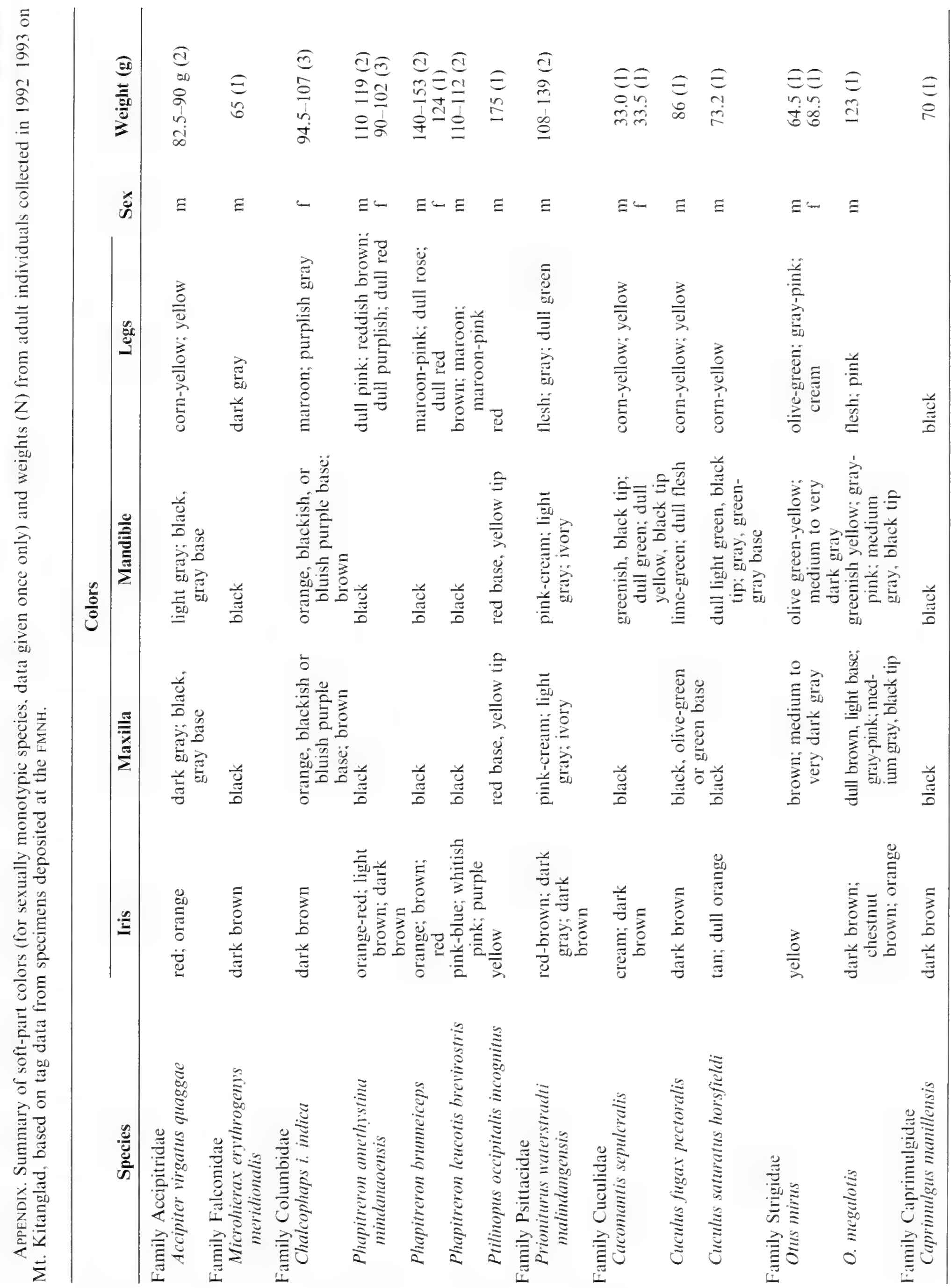




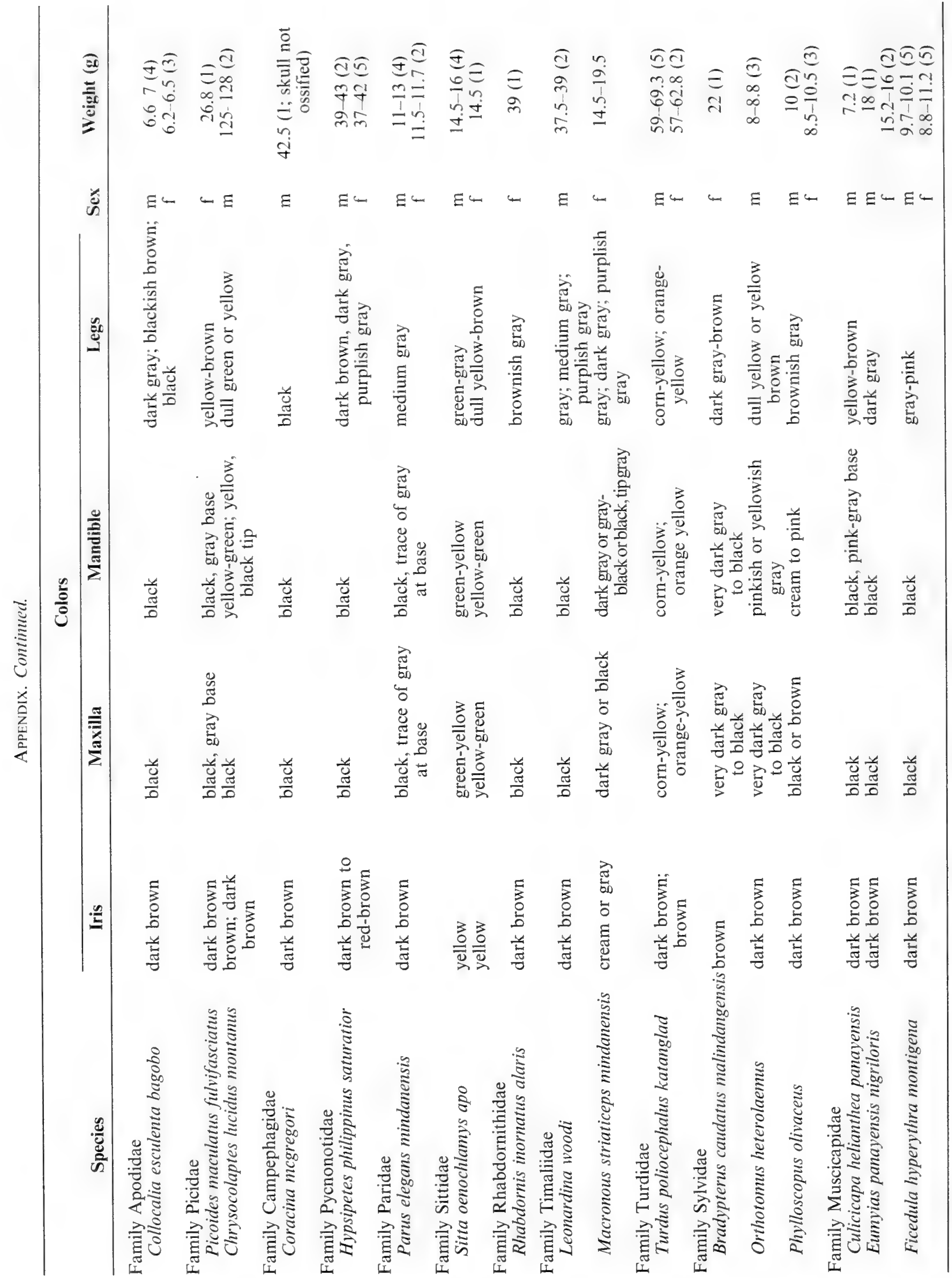




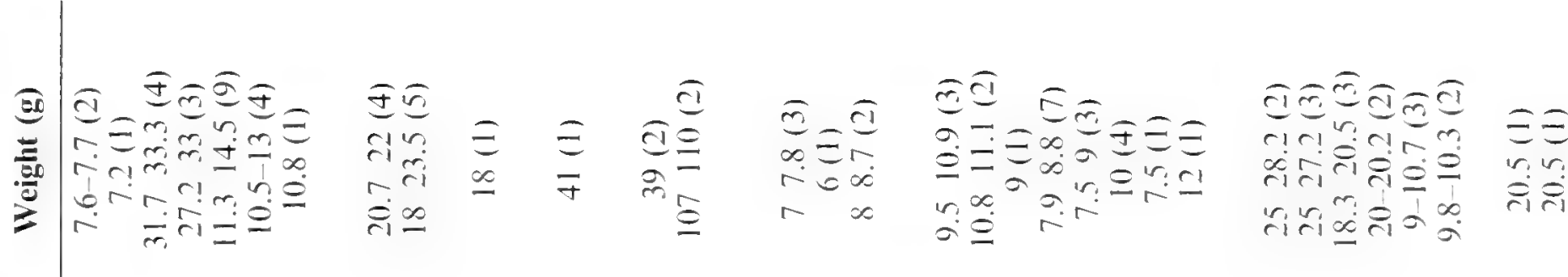

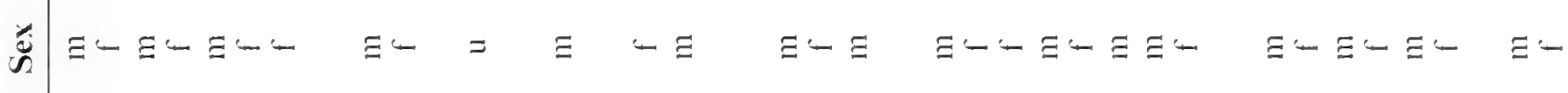

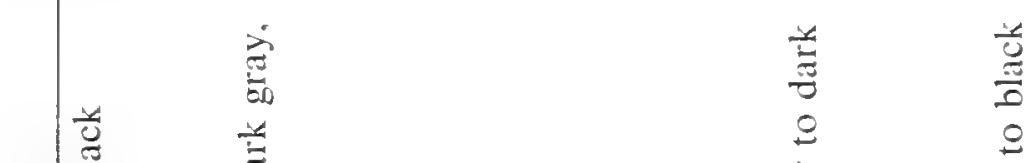

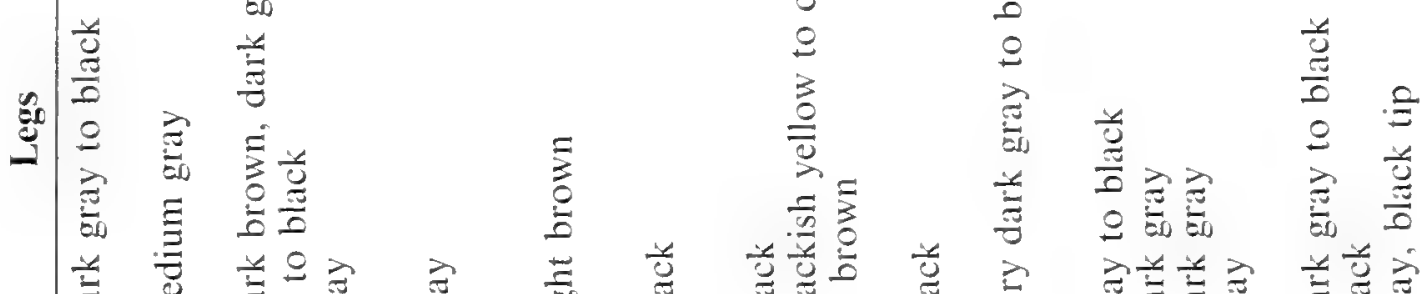

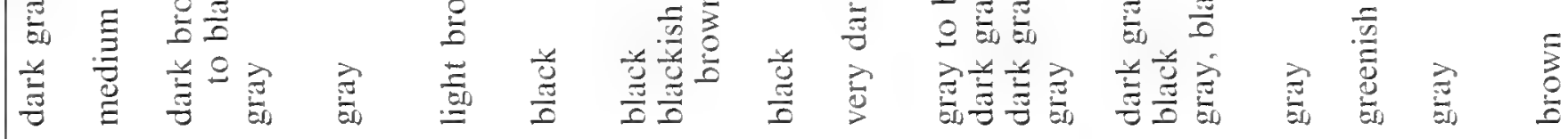
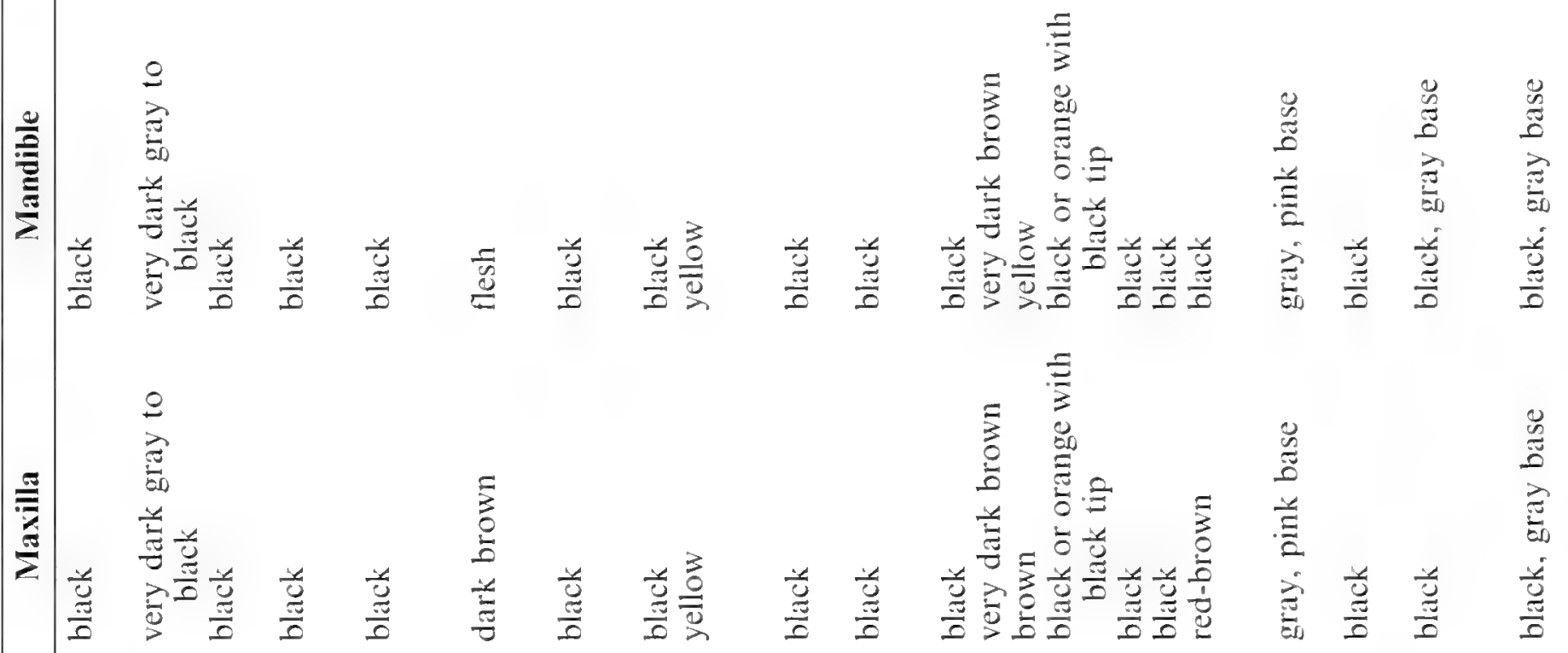

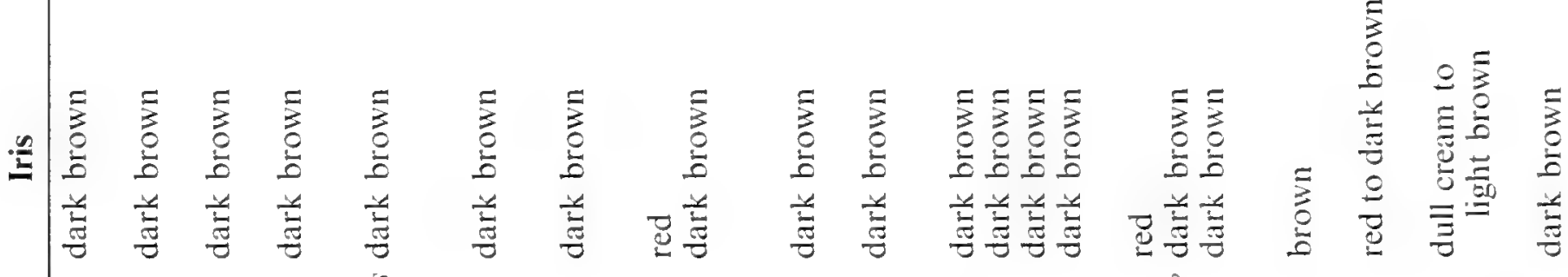

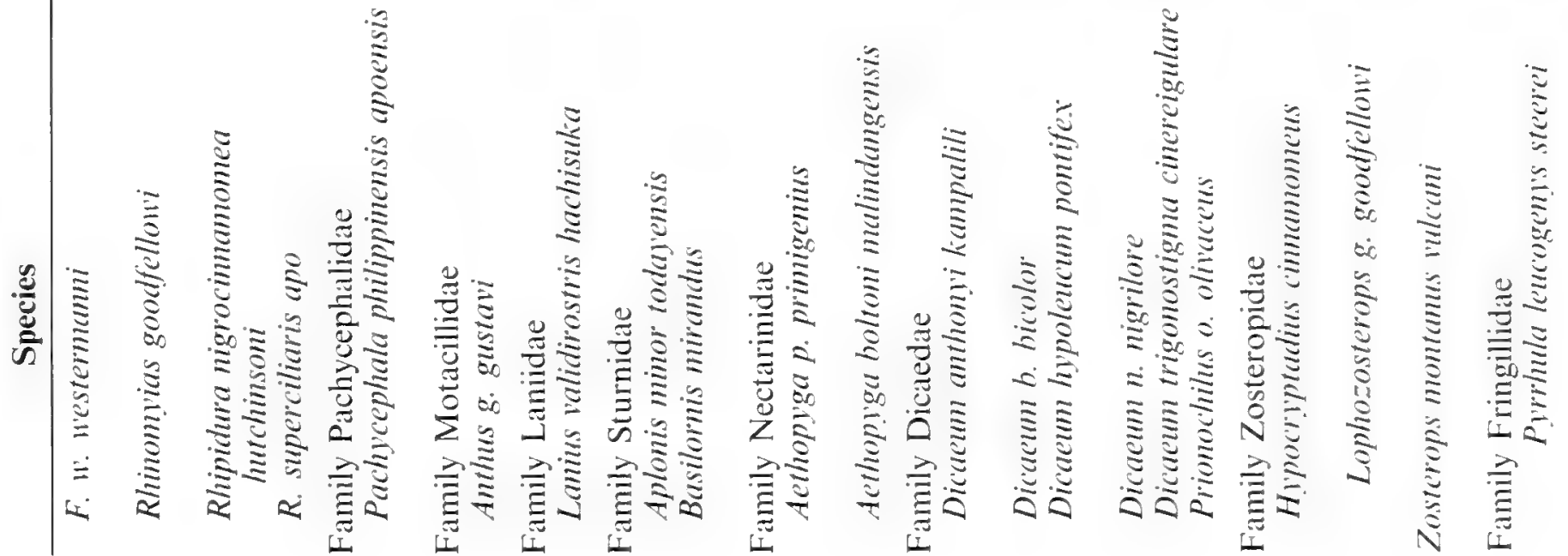




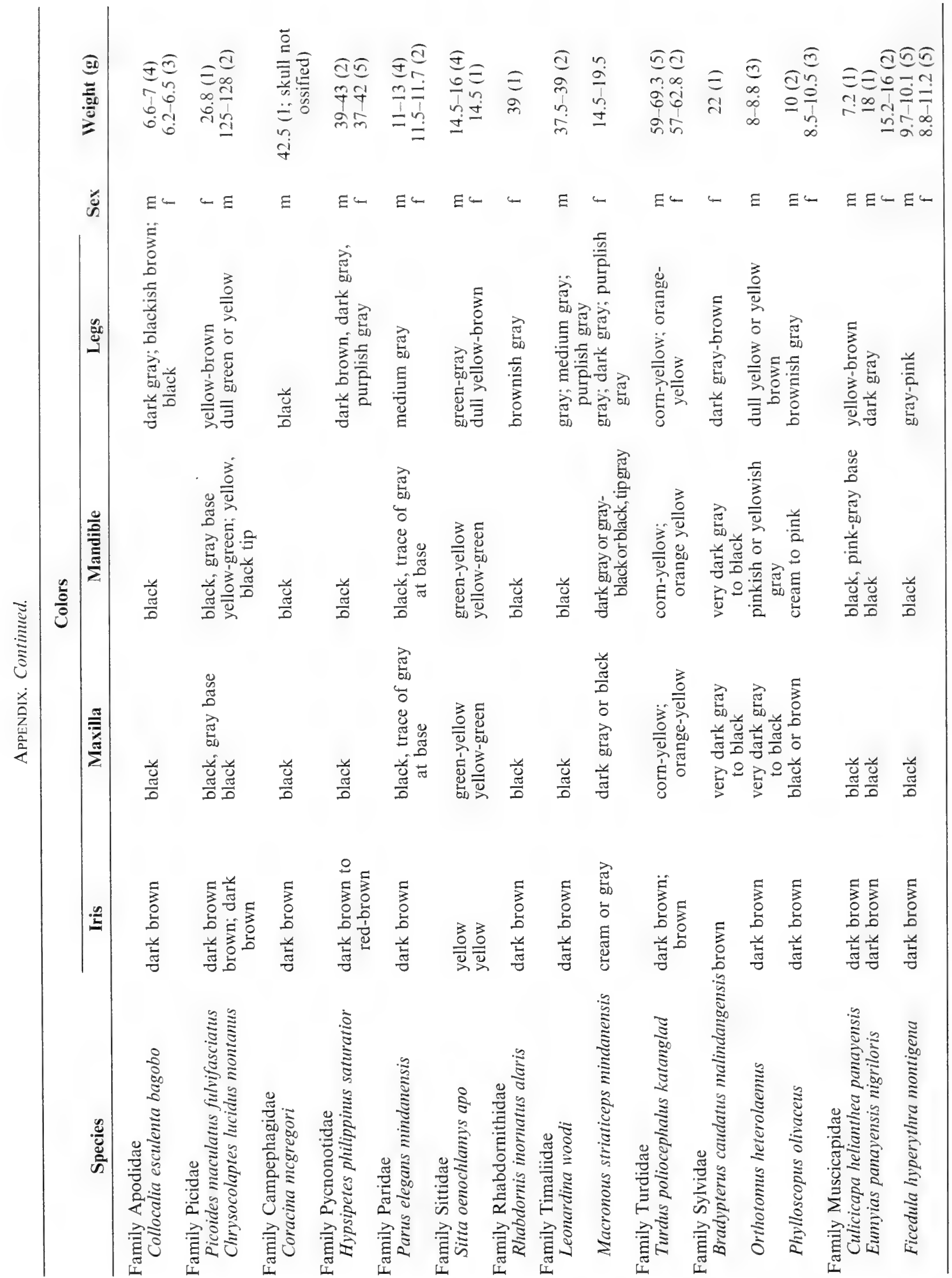




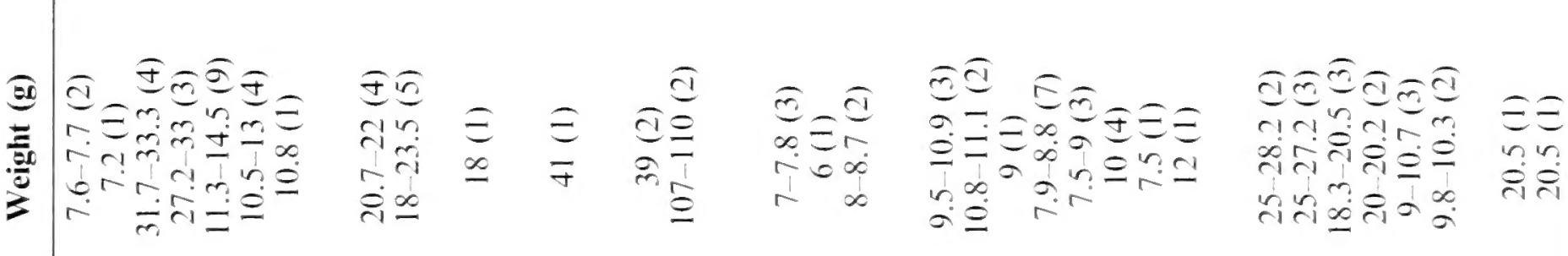

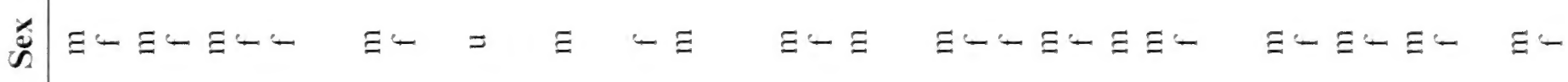

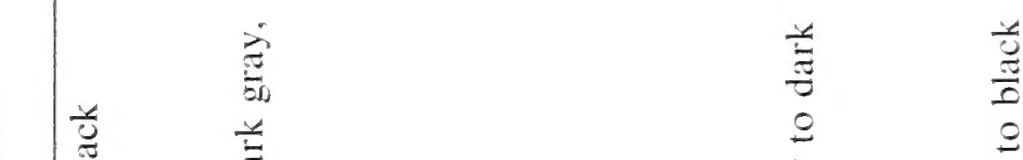

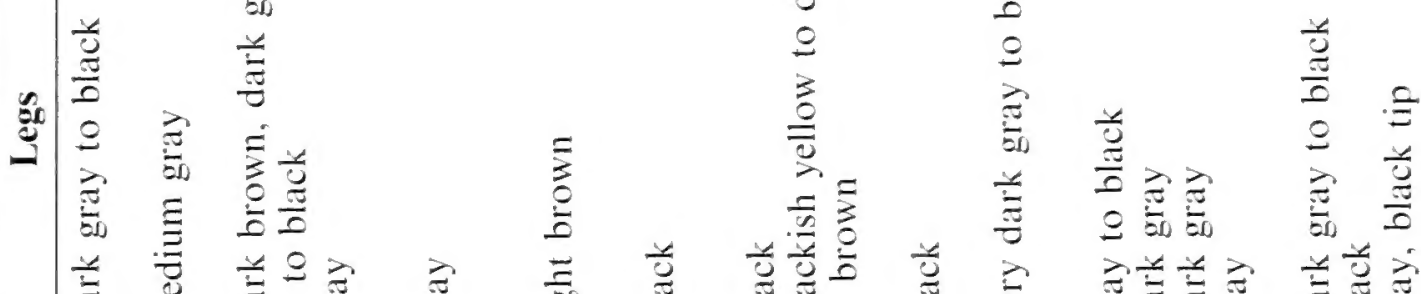

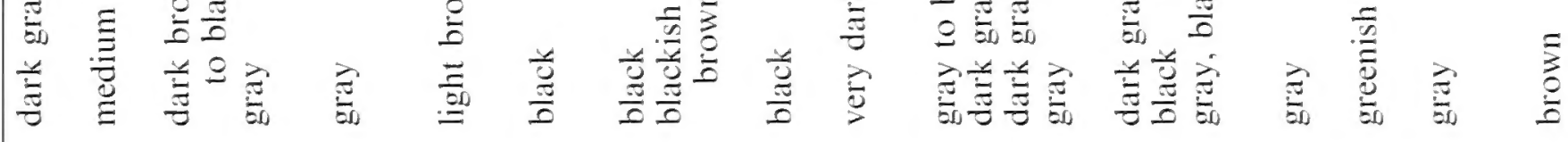

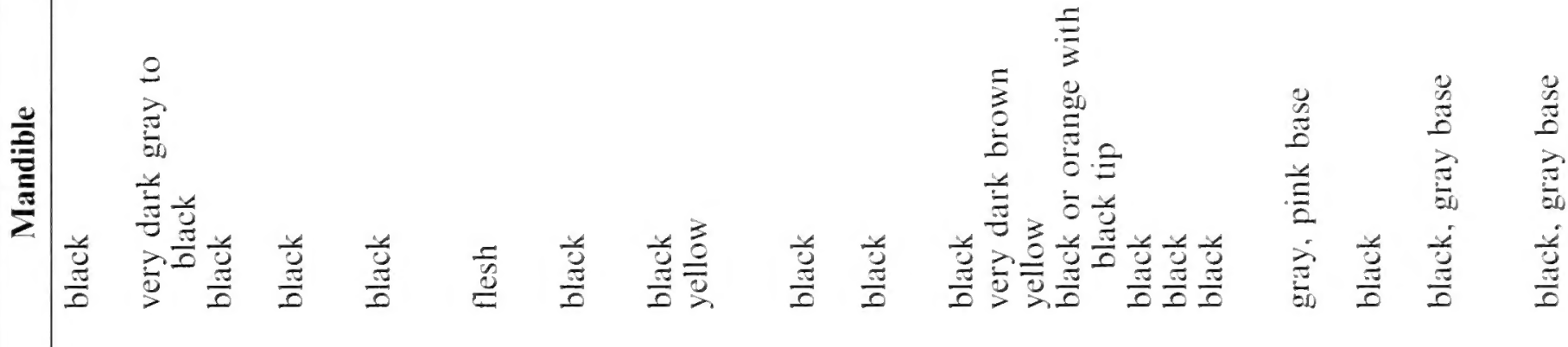

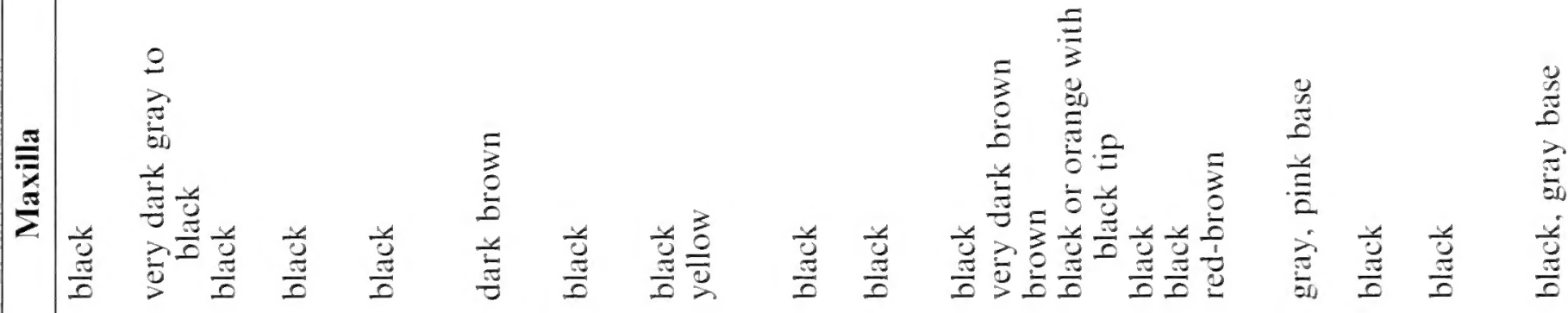

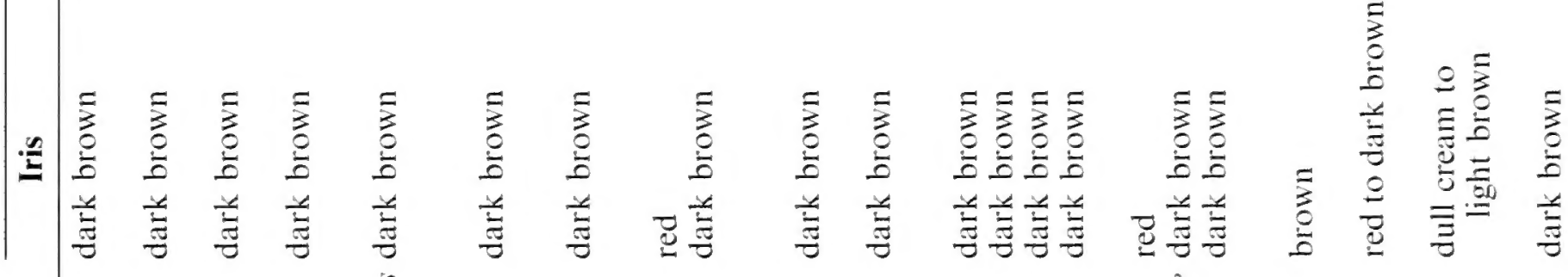

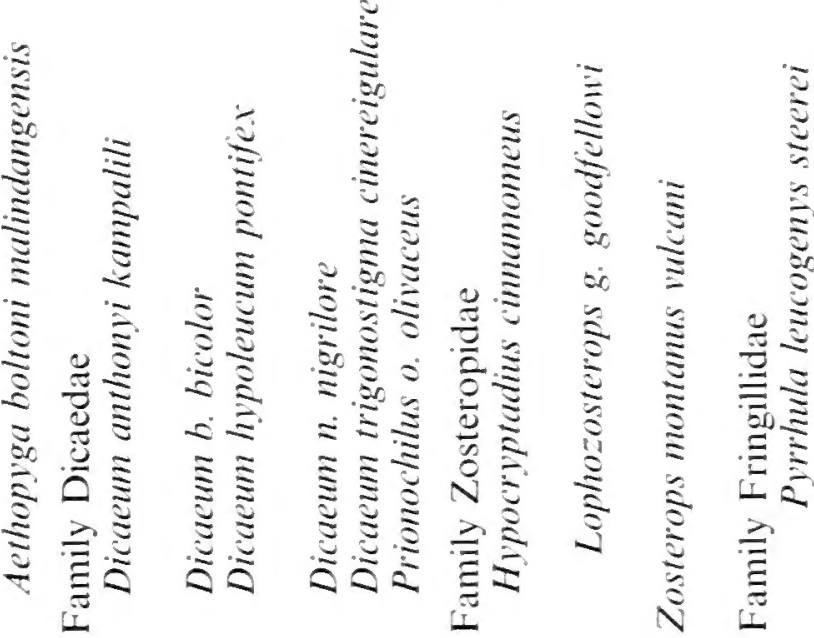






\footnotetext{
Field Muscum of Natural History
$140(0)$ South Lake Shore Drive
(hicago, Illinois $60605-2496$
Telephone: $(312) 605-7055$

Field Muscum of Natural History
$140(0)$ South Lake Shore Drive
(hicago, Illinois $60605-2496$
Telephone: $(312) 605-7055$

Field Muscum of Natural History
$140(0)$ South Lake Shore Drive
(hicago, Illinois $60605-2496$
Telephone: $(312) 605-7055$
}

(1)

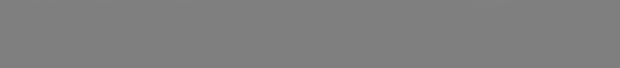

. 